\title{
THE ROLE OF RESISTANCE EXERCISE TRAINING \& IGF-1 SIGNALING IN THE AMELIORATION OF MILD COGNITIVE IMPAIRMENT IN FEMALE WISTAR RATS
}
A Dissertation presented to the Faculty of the Graduate School at the University of Missouri-Columbia

In Partial Fulfillment of the Requirements for the Degree

Doctor of Philosophy

by

\section{TAYLOR JACOB KELTY}

Dr. Frank W. Booth, PhD, Dissertation Supervisor

May 2021 
The undersigned, appointed by the dean of the Graduate School have examined the dissertation entitled:

THE ROLE OF RESISTANCE-EXERCISE TRAINING \& IGF-1 SIGNALING IN THE AMELIORATION OF MILD COGNITIVE IMPAIRMENT IN FEMALE WISTAR RATS

\author{
Presented by Taylor Jacob Kelty \\ A candidate for the degree of Doctor of Philosophy \\ and hereby certify that, in their opinion, it is worthy of acceptance
}

Frank W. Booth, Ph.D.

Nicole L. Nichols Ph.D.

Cathleen M. Kovarik, DVM, Ph.D.

Victoria Vieira-Potter, Ph.D. 


\section{DEDICATION}

This dissertation is dedicated to my remarkable parents Tom \& Deanna Kelty,

my amazing brother Conner Kelty, my incredible wife June Kelty, and my precious daughter Claire Kelty.

Without your unwavering support, I would not have made it. 


\section{ACKNOWLEDGEMENTS}

I have the deepest gratitude for my mentor and role-model Dr. Frank Booth. I would not be where I am today, without him. He has instilled in me his passion for science, which I will carry with me through the rest of my career. I have come to admire both his humbleness, honesty, and high sense of morality, which I hope has been instilled in me. No words suffice to express my appreciation for the opportunity he has given me, by allowing me to be trained by him over the last five years.

I would not only like to thank Dr. Frank Booth, but also the other members of the lab who have helped me grow both my creativity and tenacity. My thanks goes out to Thomas Childs, who has taught me much many of the molecular techniques used in this dissertation. I also want to thank other students in the lab, some of which has graduated and are perusing the next stages in their careers, and those who are still training in the Booth lab. A big thanks goes out to Dr. Greggory Ruegsegger and Dr. Kolter Grigsby who helped nourish my scientific abilities during their infancy. I also want to thank Xuangsong Mao and Nathan Kerr for supporting me throughout my dissertation process.

I would like to also thank my committee, who have all been so patient with me as I have struggled through my journey through the dissertation process. Thank you to Dr. Kovarik for teaching me how to make artificial cerebrospinal fluid, and for giving me the opportunity to assist her in the neuroanatomy lab. Thank you to Dr. Nichols, for the, what seemed to be endless, trouble-shooting for how to measure proliferation in my samples. Also big thanks to Dr. Vieira-Potter for all the help with the metabolic resistance-exercise training study. Thank you for helping the Booth lab design and measure changes in body composition after resistance-exercise training. In addition to lending me your expertise in developing my laboratory skills, your irreplaceable input on my experiments have pushed 
me to further my own critical thinking skills that will be invaluable as I peruse the next stages of my career.

Thank you to the Department of Psychology here at University of Missouri. Dr. Todd Schachtman and your graduate students Dr. Rachel A Richardson and Paige N Michener, who taught me how to perform the behavioral testing used in this thesis. Thank you to Dr. Dylan Olver and Dr. Christian Robert for your constructive feedback on my first manuscript.

I would like to thank both Karol Dinwiddie and Tommy Thomas who were always there when I needed them. Thank you to Karol for being my second mom during my stay in the BMS family, always reminding me when I forgot to register for classes or helping me find a room for a meeting that I should have scheduled weeks before. Thank you to Tommy, who helped me submit many grants in the Booth lab.

Many thanks go out to all the former and current graduate students, especially those whom I lived with during my graduate schooling. Thank you to Dr. Greggory Ruegsegger, Dr. Kolter Grigsby, Dr. Rayne Limb, and Dr. Brian Ruyle for putting up with living with me and for answering all my questions when we talked science. A big thanks to Scott Brown, whom was my roommate for the majority of my graduate schooling, for keeping me sane during the stressors of graduate school. To all the graduate students, thanks for making my time here more enjoyable, I will cherish the relationships I have made with all you and look forward to seeing what the future brings for all of us. I also would like to thank my wife, who has been so supportive of me. She has been there for me to lean on throughout my dissertation process. Finally, I would like to thank all of Mizzou facility and staff you have all made my transition to Columbia so much easier. Thank you to everyone for making me feel a part of the BMS family. It has been a treasure to wake up and come to work knowing there was such a supportive network around. 


\section{TABLE OF CONTENTS}

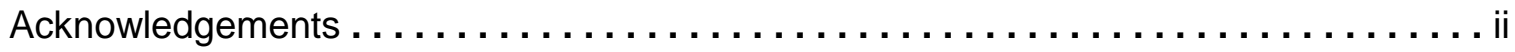

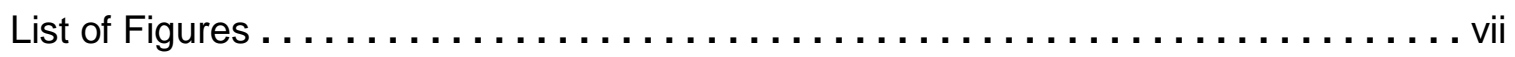

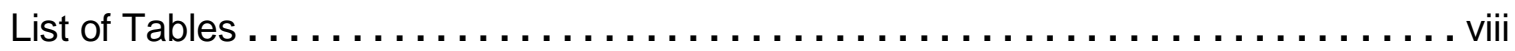

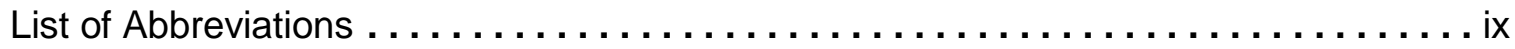

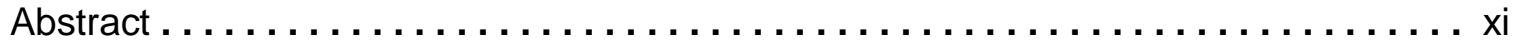

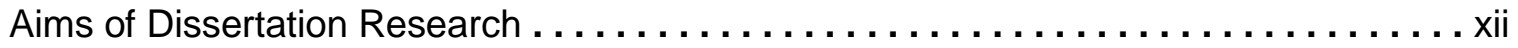

Overall objective of research $\ldots \ldots \ldots \ldots \ldots \ldots \ldots \ldots \ldots \ldots \ldots \ldots \ldots \ldots \ldots \ldots \ldots \ldots$

Overall hypothesis $\ldots \ldots \ldots \ldots \ldots \ldots \ldots \ldots \ldots \ldots \ldots \ldots \ldots \ldots \ldots \ldots \ldots \ldots \ldots \ldots \ldots$

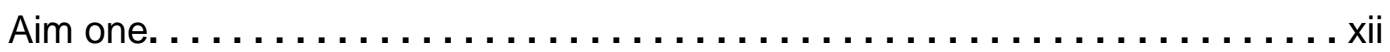

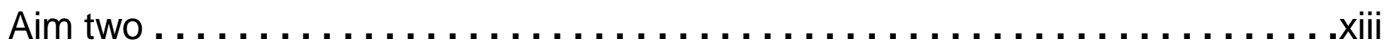

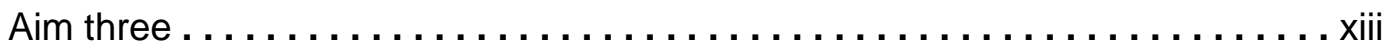

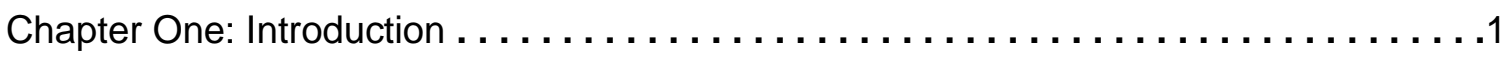

The increasing need of therapeutics for neurodegenerative dementia $\ldots 1$

Defining neurodegenerative dementia $\ldots \ldots \ldots \ldots \ldots \ldots \ldots \ldots \ldots \ldots \ldots$

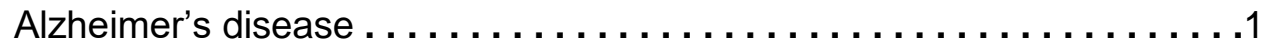

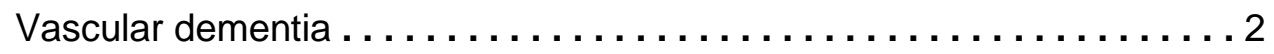

Lewy-body dementias $\ldots \ldots \ldots \ldots \ldots \ldots \ldots \ldots \ldots \ldots \ldots \ldots \ldots \ldots \ldots \ldots$

Summarizing the increasing need of therapeutics for neurodegenerative

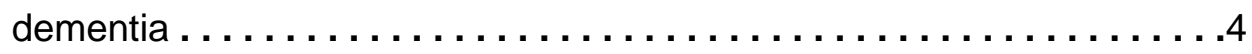

Targeting dementia early can improve patient outcomes $\ldots \ldots \ldots \ldots \ldots \ldots 4$

$\mathrm{MCl}$ is an early stage of neurodegenerative dementia $\ldots \ldots \ldots \ldots \ldots .4$

$\mathrm{MCl}$ can be a reversible precursor of neurodegenerative dementia $\ldots \ldots 5$

Early treatment of neurodegenerative dementia improves outcomes ....6

Preclinical models can be used to better understand $\mathrm{MCl} \ldots \ldots \ldots \ldots \ldots \ldots$

$\mathrm{MCl}$ prevalence, pathogenesis, and treatment $\ldots \ldots \ldots \ldots \ldots \ldots \ldots$ 
Neuroinflammation as one underlying cause of $\mathrm{MCl} \ldots \ldots \ldots \ldots \ldots 7$ LPS-induced neuroinflammation as a translation animal model of $\mathrm{MCl}$. . 9 RT can be harnessed to better understand the neuroprotective effects of IGF-1 11 Examining $\mathrm{RT}$ as a therapeutic for $\mathrm{MCl}$ in the clinical setting $\ldots \ldots \ldots 11$ RT molecular examination $\ldots \ldots \ldots \ldots \ldots \ldots \ldots \ldots \ldots \ldots \ldots \ldots \ldots \ldots \ldots \ldots$

The essential role of IGF-1 signaling in the neurodegeneration process . 13 Chapter Two: Resistance-exercise training ameliorates LPS-induced cognitive impairment concurrent with molecular signaling changes in the rat dentate gyrus $\ldots \ldots \ldots \ldots \ldots 15$

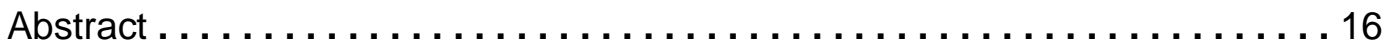

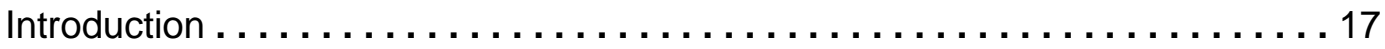

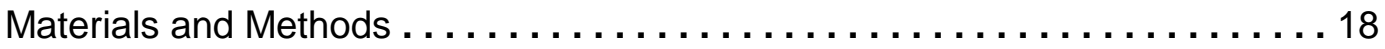

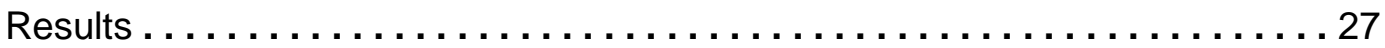

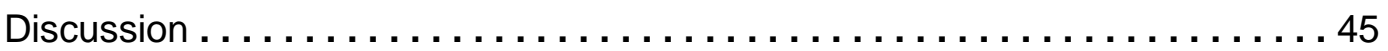

Chapter Three: Resistance-exercise training ameliorates LPS-induced neuroinflammation

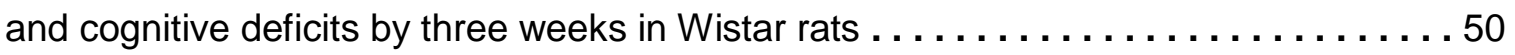

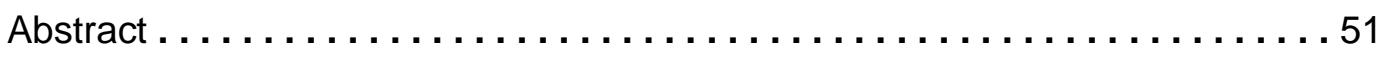

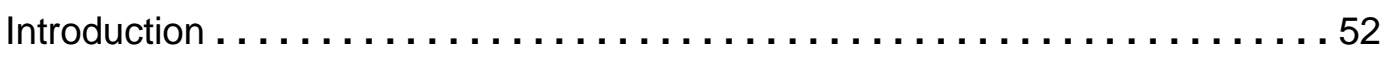

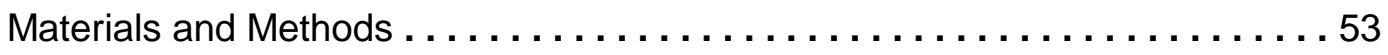

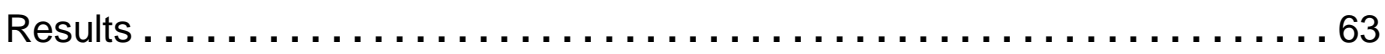

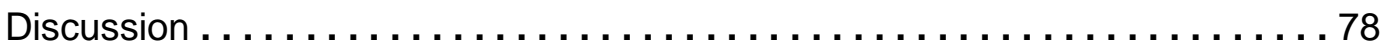

Chapter Four: Effects of resistance-exercise training and IGF-1 inhibition on cognitive performance in the ICV-LPS neuroinflammatory rat model $\ldots \ldots \ldots \ldots \ldots \ldots \ldots . \ldots 2$

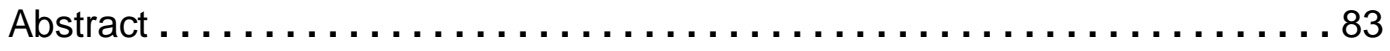

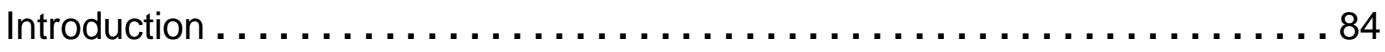

Materials and Methods $\ldots \ldots \ldots \ldots \ldots \ldots \ldots \ldots \ldots \ldots \ldots \ldots \ldots \ldots$

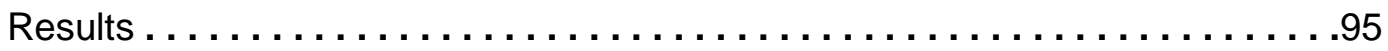




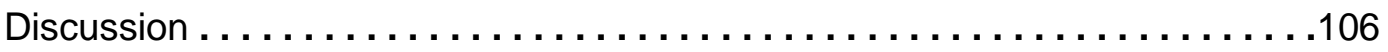

Chapter Five: Conclusions \& Future Directions $\ldots \ldots \ldots \ldots \ldots \ldots \ldots \ldots \ldots \ldots \ldots \ldots \ldots \ldots$

Twelve accumulated minutes of RT in the form of ladder climbing improves multiple forms cognition under physiological and pathological conditions in Wistar rats . 112 RT increases IGF-1R and subsequent downstream signaling in the dentate gyrus

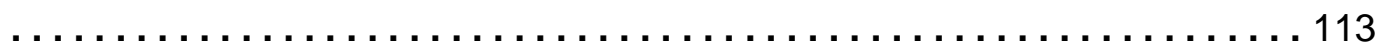

RT decreases LPS-induced neuroinflammation in the dentate gyrus in a delayed

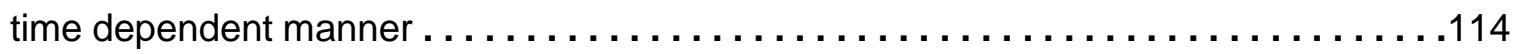

IGF-1 signaling reduces neuroinflammation in the dentate gyrus $\ldots \ldots \ldots \ldots 116$ Neuroinflammation is sufficient to cause cognitive impairment, but reduced neuroinflammation may not necessary to restore cognition $\ldots \ldots \ldots \ldots \ldots \ldots$ Limitations regarding IGF-1 signaling and cognition to be addressed in future

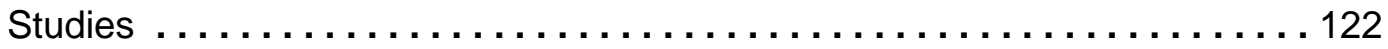

Future studies and concluding remarks $\ldots \ldots \ldots \ldots \ldots \ldots \ldots \ldots \ldots \ldots \ldots \ldots \ldots \ldots \ldots$

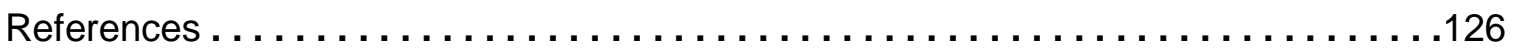

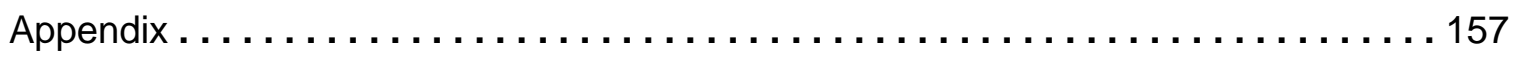

Appendix A: Abstracts from first authored original research manuscripts . . . . 157 Appendix B: Abstracts from co-authored original research manuscripts . . . . 158 Appendix C: Presented abstracts outside of dissertation topics . . . . . . . 162 Vita . . . .166 


\section{LIST OF FIGURES}

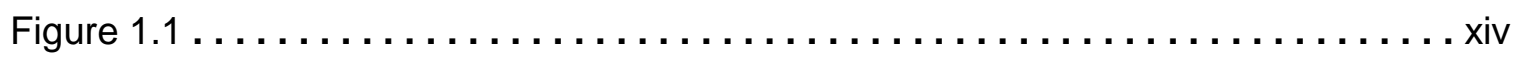

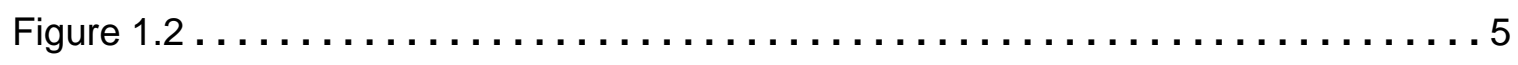

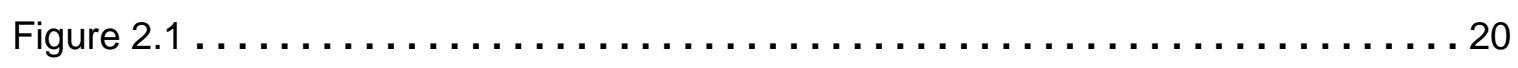

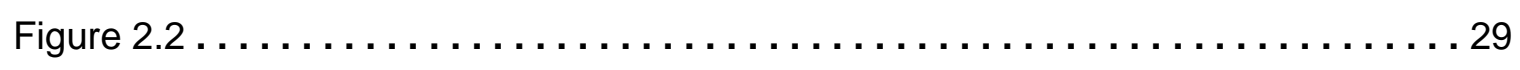

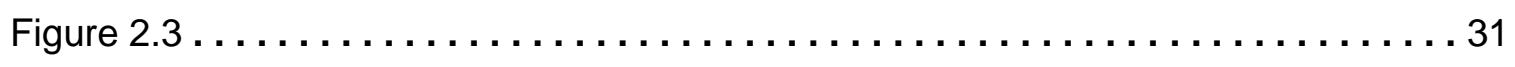

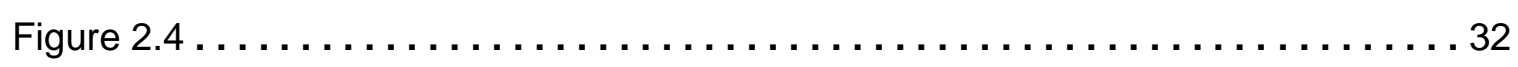

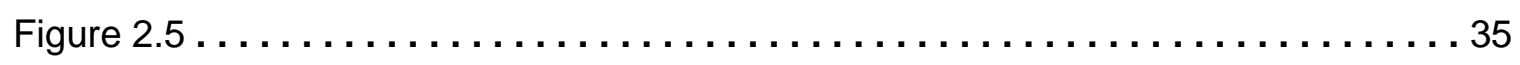

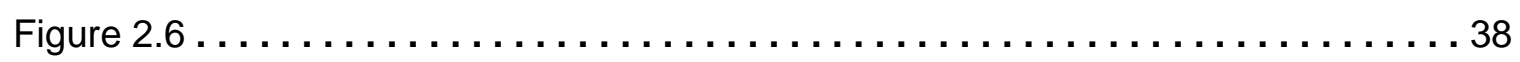

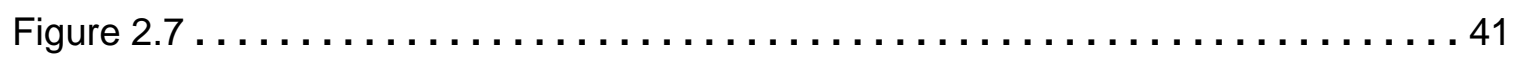

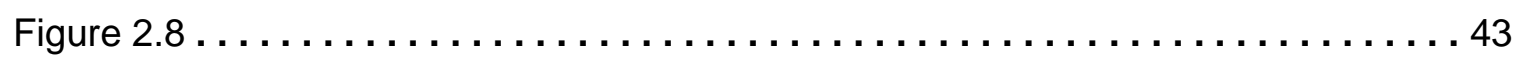

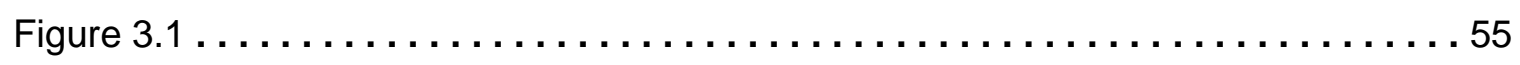

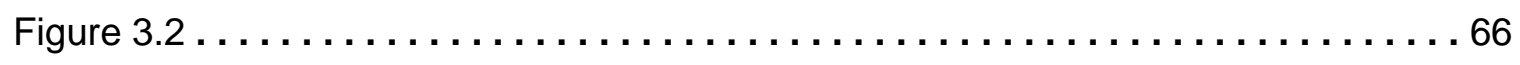

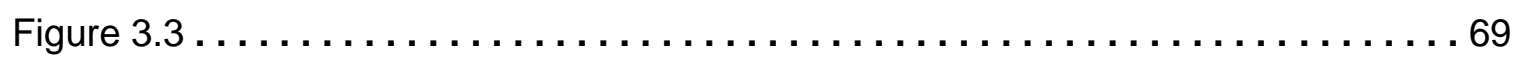

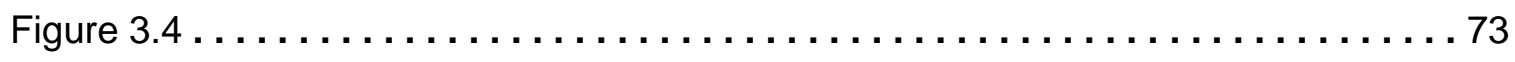

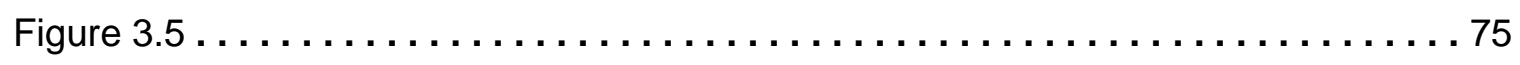

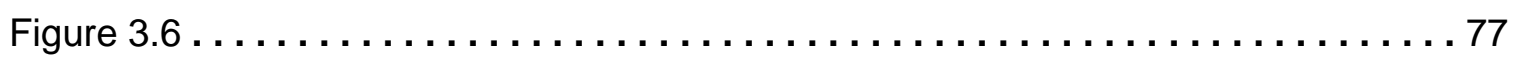

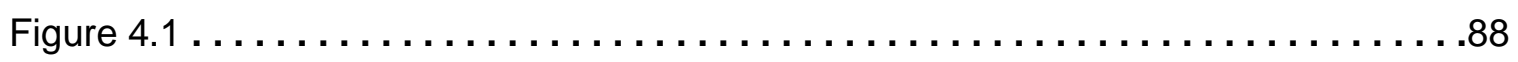

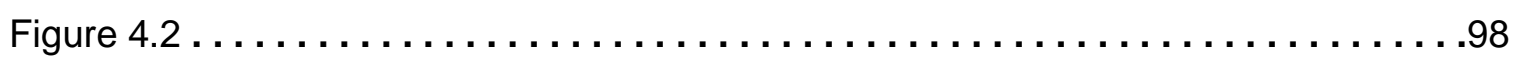

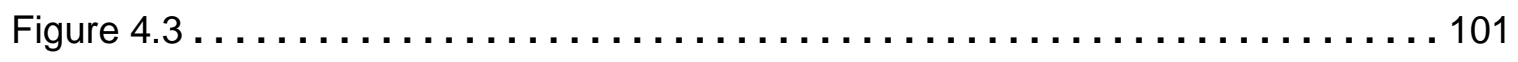

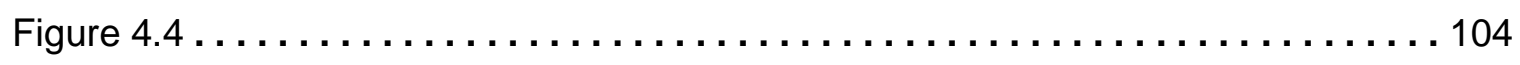

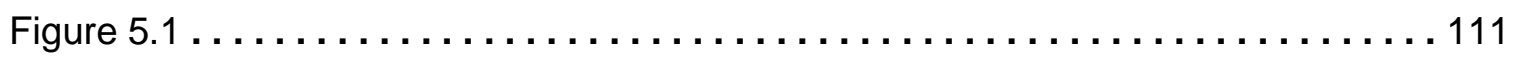




\section{LIST OF TABLES}

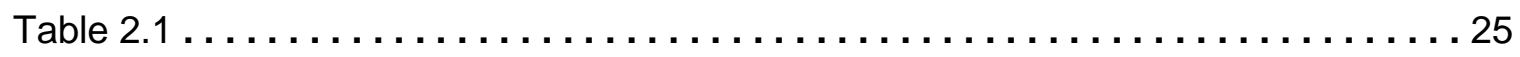

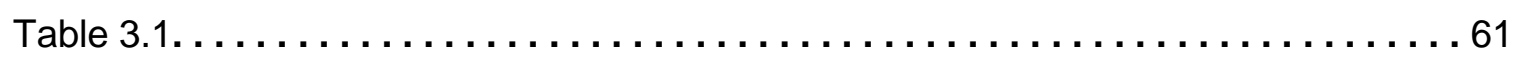

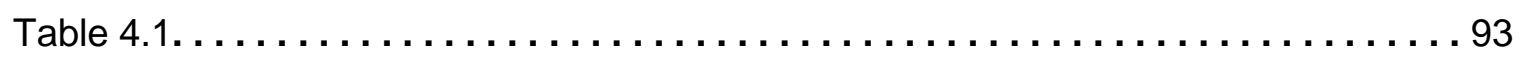




\section{LIST OF ABBREVIATIONS}

\begin{tabular}{|c|c|}
\hline$A D$ & Alzheimer's Disease \\
\hline AKT & Protein Kinase B \\
\hline BDNF & Brain-Derived Neurotrophic Factor \\
\hline DEXA & Dual-Energy X-Ray Absorptiometry \\
\hline ERK & P44/42 Mitogen-Activated Protein Kinase \\
\hline FDA & Food And Drug Administration \\
\hline GAPDH & Glyceraldehyde 3-Phosphate Dehydrogenase \\
\hline GFAP & Glial Fibrillary Acidic Protein \\
\hline GSK-3 $\beta$ & Glycogen Synthase Kinase $3 \beta$ \\
\hline ICV & Intracerebroventricular \\
\hline IGF-1 & Insulin-Like Growth Factor -1 \\
\hline IGF-1R & Insulin-Like Growth Factor -1 Receptor B \\
\hline IL & Interleukin \\
\hline LBD & Lewy-Body Dementias \\
\hline LPS & Lipopolysaccharide \\
\hline $\mathrm{MCl}$ & Mild Cognitive Impairment \\
\hline $\mathrm{p}-\mathrm{AKT}$ & Phospho-Protein Kinase B \\
\hline PCNA & Proliferating Cell Nuclear Antigen \\
\hline$p-E R K$ & Phospho-P44/42 Extracellular Signal-Regulated Kinases \\
\hline PET & Positron Emission Tomography \\
\hline pGSK & Phospho-Glycogen Synthase Kinase three Beta \\
\hline \multicolumn{2}{|c|}{ p-IGF-1R Phospho-Insulin-Like Growth Factor one Beta Receptor } \\
\hline PPI & Picropodop \\
\hline
\end{tabular}




$\begin{array}{ll}\text { RT } & \text { Resistance Exercise Training } \\ \text { SED } & \text { Sedentary } \\ \text { SYN1 } & \text { Synapsin } \\ \text { TNF-a } & \text { Tumor Necrosis Factor-Alpha } \\ \text { VaD } & \text { Vascular Dementia } \\ \text { VEH } & \text { Vehicle }\end{array}$




\title{
THE ROLE OF RESISTANCE EXERCISE TRAINING \& IGF-1 SIGNALING IN THE AMELIORATION OF MILD COGNITIVE IMPAIRMENT IN FEMALE WISTAR RATS
}

\author{
Taylor Jacob Kelty \\ Dr. Frank W. Booth, Dissertation Supervisor
}

\begin{abstract}
Mild cognitive impairment increases risk for dementia. One therapeutic intervention that is reported to ameliorate cognition in patients with mild cognitive impairment is resistance exercise. However, the underlying neuro-molecular mechanisms behind resistance exercise are largely unknown. To better understand the underlying neuro-molecular mechanisms, I established a preclinical model of resistance-exercise training and mild cognitive impairment based off the literature. Ladder climbing in female Wistar rats let to similar increases in muscle mass and strength as those observed in humans, while intracerebroventricular injections of lipopolysaccharides (LPS) induced neuroinflammation and cognitive deficits, which resembled clinical mild cognitive impairment. Resistance-exercise training restored cognitive function, increased IGF-1R phosphorylation and subsequent downstream signaling, and ameliorated neuroinflammation. Selective IGF-1R inhibition in the dentate gyrus, with picropodophyllin, had differential effects on cognition dependent on training status and type of cognition measured. Collectively, these studies indicate that although resistance training and IGF1 signaling both ameliorate LPS-induced neuroinflammation, IGF-1 signaling during resistance-exercise training, but is only necessary for its ability to ameliorate mild cognitive impairment after a single workout. Furthermore, we made a novel discovery where dentate gyrus IGF-1R inhibition can improve specific forms of cognition. These findings reveal the complexity of IGF-1 signaling and open new areas of study that could offer insights for the treatment of mild cognitive impairment.
\end{abstract}




\section{Aims of Dissertation Research}

\section{Overall objective of research}

The overall objective of the studies outlined below was to determine if IGF-1 signaling is one molecular mechanism for the neuroprotective effects of RT. In addition to establishing one possible molecular mechanism in which $\mathrm{RT}$ may be able to treat $\mathrm{MCl}$, the studies performed provide a framework for future studies to elucidate which neuroprotective benefits of IGF-1 are key for treatment of $\mathrm{MCl}$ (Fig. 1.1).

\section{Overall hypothesis}

IGF-1 signaling constitutes one molecular mechanism by which RT is able to exert neuroprotective effects resulting in amelioration of $\mathrm{MCl}$.

\section{Aim one:}

Animal studies suggests RT is able to enhance spatial memory associated with increased IGF-1 signaling and synaptic plasticity. In humans, RT has been shown to improve cognition in patients with $\mathrm{MCl}$ associated with increased IGF-1 in the blood. However, studies examining the casual relationship of the neuro-molecular adaptions that occur with RT during disease pathology are lacking. Therefore, the goal of aim one was to determine the role of $\mathrm{RT}$ in $\mathrm{MCl}$. Aim one can be subdivided into three desired outcomes.

Outcome \#1: Determine if the ladder-climbing model is model of RT is sufficient to ameliorate LPS-induced $\mathrm{MCl}$ in rats

Outcome \#2: Determine if RT is sufficient to increase proliferation markers, synaptic plasticity markers, and IGF-1 signaling in the dentate gyrus 
Outcome \#3: Determine if 1, 3, or 18 RT workouts is required to increase IGF-1R and downstream signaling in the dentate gyrus

\section{Aim two:}

Clinical evidence demonstrate that RT can exert anti-inflammatory effects, while preclinical studies suggest IGF-1 can be anti-inflammatory. Therefore, the goal of study two (chapter three) was to assess the neuro-molecular and cognitive adaptions that occur after three weeks RT during neuroinflammation. Aim two was subdivided into three desired outcomes.

Outcome \#1: Evaluate the effects of three weeks RT on markers of neuroinflammation in the dentate gyrus after LPS injections

Outcome \#2: Evaluate the effects of on both IGF-1 protein and transcriptional changes after three weeks RT and/or LPS injections

Outcome \#3: Evaluate the effects of three weeks RT on multiple indiciees of cognition (spatial memory, recognition memory, cognitive flexibility)

\section{Aim three:}

Based on the literature, IGF-1 signaling has been posited as a potential mechanism for RT-induced amelioration of $\mathrm{MCl}$. Therefore, the goal of aim three (chapter 4) was to mechanistically examined the effect IGF-1R inhibition during RT after LPS injections. Aim three could be subdivided into three desired outcomes.

Outcome \#1: Assess changes in IGF-1R signaling after IGF-1R inhibition 
Outcome \#2: Assess molecular markers of neuroinflammation after IGF-1R inhibition

Outcome \#3: Assess cognitive changes after IGF-1R inhibition

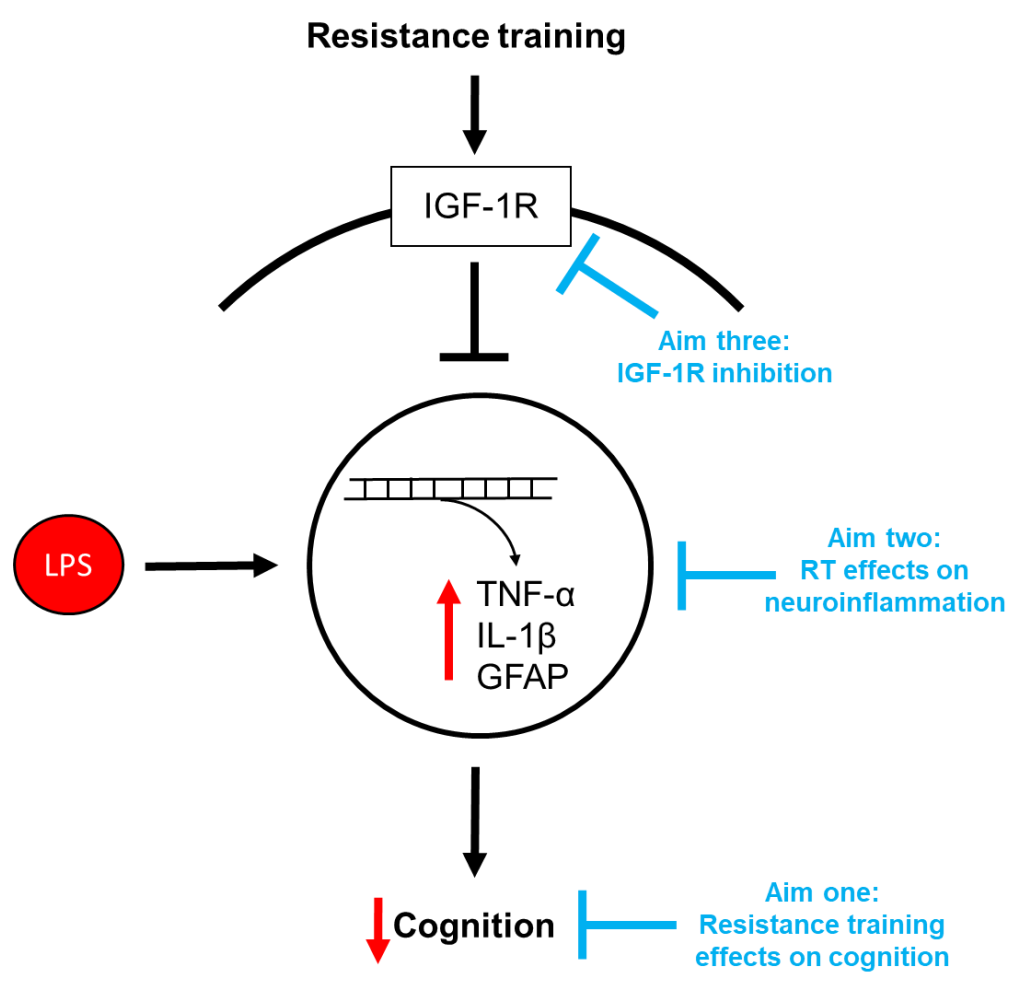

Overview of dissertation hypotheses

Aim one:

$\mathrm{RT}$ is able to ameliorate LPS-induced $\mathrm{MCl}$

\section{Aim two:}

$\mathrm{RT}$ is able to ameliorate LPS-induce neuroinflammation

Aim three:

IGF-1 inhibition during RT will attenuate the amelioration of $\mathrm{MCl}$ and neuroinflammation

Figure 1.1 Overall hypothetical model and mechanism tested for dissertation thesis. 


\section{Chapter One: Introduction}

\section{The increasing need of therapeutics for neurodegenerative dementias}

\section{Defining neurodegenerative dementia}

Neurodegenerative dementia is often classified as an irreversible and significant decline from one's previous level of cognition that interferes with daily function (1). Neurodegenerative dementia prevalence increases with age where the causes of cognitive decline can be attributed to multiple neurodegenerative diseases $(1,2)$. Therefore, it may be more accurate to think of dementia as an acquired syndrome, rather than a specific disease. The most common neurodegenerative diseases that cause neurodegenerative dementia include Alzheimer's disease (AD), vascular dementia (VaD), and the dementia with Lewy-bodies (1). To gain a better understanding of each neurodegenerative disease and the therapies used to combat them, this section will examine each of the aforementioned conditions separately to expose the growing need for novel therapeutics to treat neurodegenerative dementia.

\section{Alzheimer's disease (AD)}

$A D$ is the most common cause of dementia, accounting for over $70 \%$ of dementia cases in the United States (3). Although many theories on the pathogenesis of AD exist (4), the classical hallmarks of $A D$ are extracellular plaques of $\beta$-amyloid peptide and neurofibrillary tangles, which lead to the degradation and loss of neurons attributing to the cognitive decline (5). Current medications for AD include cholinesterase inhibitors or N-methyl-daspartate receptor antagonist. These medications improve cognitive and behavioral symptoms for a transient period (6 months to several years), but they do not slow the 
progression of $A D(6,7)$. To date, no cure for $A D$ exists $(7)$, with cases of $A D$ in the United States being predicted to more than double from approximately five million cases in 2008 to 13.8 million by 2050 (8). Taken together, the above suggest a growing need for novel therapeutics to treat $A D$.

\section{Vascular dementia (VaD)}

$\mathrm{VaD}$ is the second most common cause of dementia accounting for $17.4 \%$ of dementia cases in the United States (3). Although the intricacies of VaD require further study (9), the basic principal of $\mathrm{VaD}$ seems straight forward. In $\mathrm{VaD}$, hypertension, hypocholesteremia, myocardial infarct and other hereditary non-amyloid or amyloid angiopathies cause progressive cerebrovascular pathology resulting in a hypoxic environment that leads to a functional loss of neurons $(9,10)$. Clinical trials have begun to explore possible therapeutics for $\mathrm{VaD}$ including cholinergic stimulants, vasodilators, platelet aggregation inhibitors, yet as of now there are no food and drug administration (FDA) approved drug for treatments for $\mathrm{VaD}(11-14)$. VaD prevalence is predicted to rise due to the steady rise in the population suffering from its risk factors such as hypertension and cardiac disease in the United States, which suggest an increasing need for therapeutics to treat $\mathrm{VaD}$.

\section{Lewy-body dementias (LBD)}

Parkinson's disease dementia and dementia with Lewy-bodies together comprise the Lewy-body dementias (LBD) (15). Along with normal pressure hydrocephalus, frontal lobe dementia, alcoholic dementia, and traumatic brain injury, LBD account for the remaining $12.7 \%$ cases of dementia (3). Dementia with Lewy-bodies develops from the formation of Lewy-bodies containing aggregates of the misfolded protein, $\alpha$-synuclein $(16,17)$. Lewy- 
body formation causes neurodegeneration triggering cognitive decline (17). Parkinson's disease dementia is associated with neurodegeneration in the substantia nigra, an area of the brain that constitutes primarily dopaminergic neurons $(18,19)$, and is often observed with $\alpha$-synuclein protein aggregation (16). The loss of these neurons compromises the brain's ability to control movement (19), which often further develops to include cognitive deficits (20). Considering the two syndromes share many neurobiological similarities, some researchers have questioned whether Parkinson's disease dementia and dementia with Lewy-bodies are the same entity (21). Additionally, because of the overlap between the two syndromes, dementia with Lewy-bodies treatments can be relatively similar (22). One of the most widely used drug therapies for Parkinson's disease dementia and dementia with Lewy-bodies, levodopa (22-24), is a derivative of dopamine. Levodopa increases dopamine synthesis to improve clinical outcomes of Parkinson's disease dementia and dementia with Lewy-bodies $(22,24,25)$. Although levodopa treatment yields small benefits in patient-rated mobility scores, neuroimaging data suggest either that levodopa accelerates the loss of nigrostriatal dopamine nerve-terminals or alters the dopamine transporter, which give rise to its potential negative long-term side effects (26). Therefore, the usage of levodopa as a long-term treatment remains uncertain. From 1990 to 2015 , the number of people with Parkinson disease doubled to over six million. Driven principally by aging, this number is projected to double again to over 12 million by 2040 (27), which could lead to more cases of LBD. Due to the complexity of the neurological condition, LBD is considered irreversible and currently has no cure (28), supporting the necessity of new therapeutics for LBD. 


\section{Summarizing the increasing need of therapeutics for neurodegenerative dementia}

Neurodegenerative dementia is rapidly increasing worldwide (2). Current treatments for neurodegenerative dementia focus on alleviating behavioral symptoms instead of attacking the key mechanisms that cause the initial neuronal loss $(6,7,26)$. Due to the complexity of the mechanisms that cause this loss of neurons, neurodegenerative dementias are considered irreversible and currently have no cure $(7,14,28)$. However, some research suggests that if dementia is targeted early enough it might be possible to reverse (29). The next section will examine the literature on mild cognitive impairment $(\mathrm{MCl})$ as a way to target dementia early, which will provide the framework of my dissertation thesis.

\section{Targeting dementia early can improve patient outcomes}

\section{$\mathrm{MCl}$ is an early stage of neurodegenerative dementia}

$\mathrm{MCI}$ has been theorized as the transition state between normalcy and dementia (30). One study found when assessing the rate of progression of $\mathrm{MCl}$ to dementia, $28 \%$ of patients diagnosed with $\mathrm{MCl}$ were diagnosed with dementia within three years (31). Another study found $38.1 \%$ of women and $30.4 \%$ of men that had been diagnosed $\mathrm{MCl}$ were diagnosed with dementia within a five-year follow-up period (32), suggesting a strong association between $\mathrm{MCl}$ and dementia. Although $\mathrm{MCl}$ has been shown to be an early stage for $\mathrm{AD}$, $\mathrm{VaD}$, and LBD (33), much of the research has focused on the association between $\mathrm{MCl}$ and $\mathrm{AD} . \mathrm{MCl}$ has been hypothesized to represent the earliest symptomatic stage of $A D$ (34). Further examination of AD shows, epidemiological, neuropathological, and clinical

trial research on $\mathrm{MCI}$ has become the focus of many researchers now that patients who meet the criteria for $\mathrm{MCl}$ can be differentiated from healthy control subjects and those with AD $(35,36)$. Researchers have found by using multimodal and multiscale deep neural 
network classification based on neuroimaging scans, it is possible to accurately identify individuals with $\mathrm{MCl}$ who will convert to $\mathrm{AD}$ (86.4\% combined accuracy for conversion

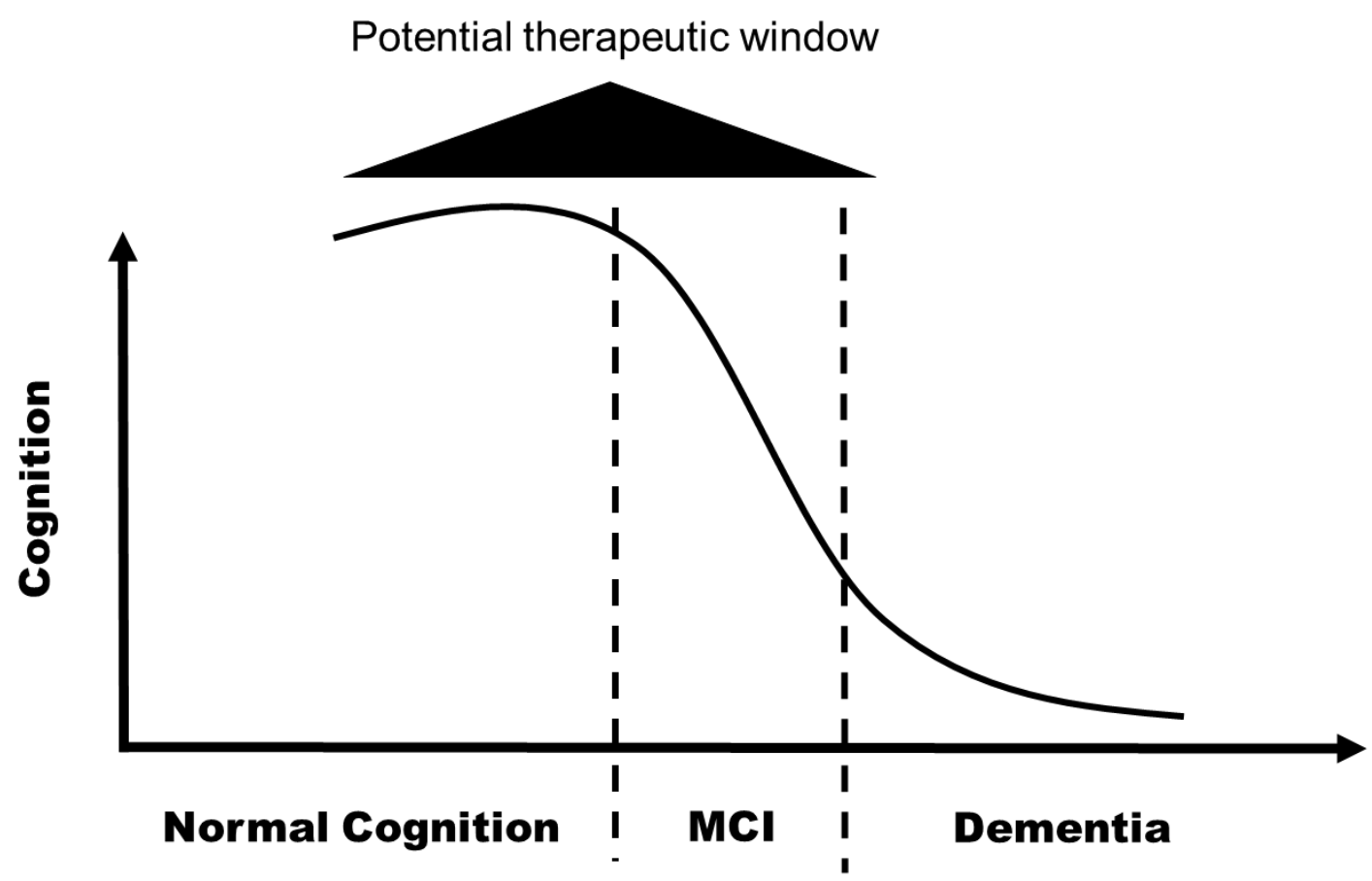

within one-to-three years) (37). This study advocates, with the use of advanced modeling, it is now possible to predict whether clinical symptoms of cognitive decline will progress into neurodegenerative dementia. Taken together this literature supports the shift to treating $\mathrm{MCl}$, which is potentially reversible (38), instead of neurodegenerative dementias which are considered irreversible (1) (Fig. 1.2).

Figure 1.2 Schematic representing the potential therapeutic window between normal cognition and dementia.

\section{$\mathrm{MCl}$ can be a reversible precursor of neurodegenerative dementia}

Similar to neurodegenerative dementia (39), there are many low cost lifestyle interventions that can potentially improve $\mathrm{MCl}$ outcomes $(40,41)$. However, what separates $\mathrm{MCl}$ from neurodegenerative dementia is its ability for complete reversion back to normal cognition 
(42). Data suggest individuals most likely to revert from $\mathrm{MCl}$ back to normal cognition are those who continue to engage in specific lifestyle activities like sports, hobbies, or fieldwork (42). While some studies suggest no benefit or only a trend in decreased risk of dementia from physical activity $(43,44)$, other studies suggest physical activity may reduce the risk of developing dementia $(45,46)$. For example, one study that accessed $176 \mathrm{MCl}$ patients over 3-years found subjects with the highest third of physical activity scores had the lower hazard ratio (HR 0.44) for developing dementia (45). Another study examining 308 patients with $\mathrm{MCl}$ found individuals, which underwent a combined physical and cognitive activity program (90-minute sessions for 40 weeks) improved or maintained cognition, suggesting not only a delay in $\mathrm{MCl}$, but also a potential reversal of $\mathrm{MCl}$ from an activity program (46). Despite evidence suggesting $\mathrm{MCl}$ as a reversible precursor for neurodegenerative dementia, there is currently no FDA approved drug for treating solely $\mathrm{MCl}(47)$.

\section{Early treatment of neurodegenerative dementia improves outcomes}

Detecting the causes of neurodegenerative dementias early can be beneficial, as early treatment has been shown to slow/reverse progression through $\mathrm{MCl}$ to pathologies for $A D, V a D$, and LBD. For example, Levey et al. concluded after their comprehensive search of the literature that cholinesterase inhibitors associated with a significantly lower rate of progression to $\mathrm{AD}$ compared with placebo during the first 12 months of treatment in $\mathrm{MCl}$ patients, but had little effect in patients who already had AD (48). A 2019 Nature paper that examined 48 individuals with varying degrees of $A D$ pathology using single-cell transcriptomics revealed the strongest disease-associated changes occur early on in the pathological progression (49). Later pathological progression of AD displayed a common global stress response across all cell-types, advocating for attention to be more focused

on the earlier stage of $A D$, instead of in the later stages of the disease. AD is not the only 
cause of neurodegenerative dementia that displays strong molecular adaptations early on, progressive pathological cortical wall thinning can be observed in vascular $\mathrm{MCl}$, the precursor to $\mathrm{VaD}(50)$. Additionally, crossing linking of $\alpha$-synuclein a pathogenic hallmark of LBD, is found in pre-clinical Parkinson's patients (51). Like AD, many treatments for vascular and LBD also focus on treatment during the early stages of the disease (52-55), further supporting early treatment, prior to overt pathology, of neurodegenerative disease for improved outcomes. Of the commonalities between $A D, V a D$, and $L B D$, one useful diagnosable trait that continues to gain support for early treatment of neurodegenerative disease is $\mathrm{MCl}(56)$. The next section will discuss how $\mathrm{MCl}$ can be better understood in order to create potential therapeutics.

\section{Preclinical models can be used to better understand $\mathrm{MCI}$}

\section{$\mathrm{MCl}$ prevalence, pathogenesis, and treatment}

Approximately $46 \%$ of people with $\mathrm{MCl}$ develop dementia within three years while only $3.3 \%$ of the population without $\mathrm{MCI}$ go on to develop dementia in that three year timespan (57). $\mathrm{MCl}$ treatment is growing increasing important considering $\mathrm{MCl}$ affects nearly $20 \%$

of people 65 years or older $(58,59)$. Although, cholinesterase inhibitors that are FDA approved to treat $A D$ have been shown to delay $A D$ in $M C I$ patients (48). No FDA approved drug exists that specifically targets and prevent $\mathrm{MCl}$ (47), emphasizing a greater need for understanding the pathological progression of $\mathrm{MCl}$.

While the pathogenesis of $\mathrm{MCl}$ is largely unknown, there is some evidence to suggest a combination cognitive restructuring, reduced brain plasticity, increased mitochondria dysfunction, and increased inflammation contribute to the cognitive decline in $\mathrm{MCl}(60$ 62). One possible mechanism for the cognitive restructuring comes from the under 
recruitment theory which surmises that with aging neural circuits are not voluntarily used resulting in recruitment of regions not typically seen in young adults causing cognitive decline $(60,63)$. Cognitive restructuring and reduced brain plasticity is further supported by a study indicating that during the early phase of $\mathrm{MCl}$, neural reorganization helps attenuate the cognitive decline, while in later stages there is a breakdown of this brain plasticity characterizing later stages of $\mathrm{MCl}(61)$. The breakdown of brain plasticity and further decline in cognition could also be explained in part by mitochondrial dysfunction resulting in reduced neural connectivity (64). Inflammation acting directly within brain tissue to disrupt synapses, suggest neuroinflammation could provide another explanation for the reduced brain plasticity that is seen in later stages of cognitive impairment (65). Further evidence for increased inflammation contributing to $\mathrm{MCl}$ comes from studies using anti-inflammatories in $\mathrm{MCl}$ patients to reduce the risk of $\mathrm{AD}$ and Parkinson's disease (66, 67). However, anti-inflammatory drugs seem to not offer any beneficial effect when used to treat $A D(68)$, suggesting inflammation as a major driver of progression of $M C l$ to $A D$.

\section{Neuroinflammation as one underlying cause of $\mathrm{MCI}$}

The 'free-radicals theory of aging' was first published by Harmon in 1956 (69), postulating that aging, including age-associated degenerative diseases, are a result of a buildup of free radicals that harms tissues in the body (70). Considering free radicals are derived mainly from oxidative phosphorylation, which takes place in the mitochondria (70), this theory has led others to suggest mitochondrial dysfunction as a potential key initiator of neurodegeneration $(71,72)$. Conversely, due to inflammations involvement in free-radical creation (73), others suggest the start of the neurodegeneration process begins with neuroinflammation, which has been substantiated in clinical and animal research $(74,75)$.

Clinical studies using positron emission tomography (PET) scans combined with inflammatory ligands have revealed neuroinflammation as one of the earliest detectable 
biomarkers in dementia progression $(75,76)$. PET scans reveal levels of neuroinflammation were modest in $\mathrm{MCl}$ patients, while much higher in $\mathrm{AD}$ patients compared to controls, which can be correlated to a decline in cognition, suggesting a progressive increase in neuroinflammation as cognitive impairment worsens (75). Lipopolysaccharide (LPS), a component of gram-negative bacteria that targets the toll-like receptor to induce inflammation in animal models strongly implicates neuroinflammation as one of the initiators of neurodegeneration (77). Increased oxidative stress, induced mitochondria structural damage, and impaired mitochondrial bioenergetics are observed from LPS injections in the striatum (74). This mitochondrial dysfunction caused by LPSinduced neuroinflammation is associated with neurodegeneration of neurons in the striatum (74); suggesting neuroinflammation can produce increases in oxidative stress, proposing neuroinflammation as the key component in this experimental model, which starts neurodegeneration. Although the pathogenic process to $\mathrm{MCl}$ likely involves a positive feedback loop between oxidative stress, mitochondrial dysfunction, and neuroinflammation, these data suggest neuroinflammation plays a critical role for the progression of $\mathrm{MCl}$.

\section{LPS-induced neuroinflammation as a translation animal model of $\mathrm{MCl}$}

LPS has become a prominent tool in understanding the role of neuroinflammation in the pathology of $\mathrm{MCI}$ (77). LPS has likely become a widespread model to study $\mathrm{MCl}$ due the ability of LPS to mimic some of the effects seen in age-associated neurodegeneration (73). LPS is most known to cause inflammation (78), but can also cause oxidative stress, mitochondrial dysfunction, and neurodegeneration $(74,79)$, all of which can be observed in the clinical presentation of $\mathrm{MCI}$ (80-82). Additionally, LPS decreases neurotrophic factors like insulin-like growth factor one (IGF-1) mRNA and protein in post-mortem human brain sections (83). Reductions in IGF-1 levels in serum has been associated with reduced 
cognitive function in the elderly (84). A single LPS intracerebroventricular (ICV) injection or seven intraperitoneal injections over the course of one week have been shown to increase beta-amyloid levels in adult mice (78), a marker strongly associated with an increased risk for $\mathrm{MCI}(85)$. Reduced neurogenesis has also been observed from LPS injections, which resembles patients with $\mathrm{MCI}$, where neurogenic pathways are reversely associated with cognitive decline $(86,87)$. Further support of the LPS-induced MCI model comes from the endotoxin hypothesis, which suggests that endotoxins (a type of LPS), from the gut, gums, or some type of infection can increase blood endotoxin levels leading to increased neuroinflammation resulting in neurodegeneration (88). Evidence of this theory comes from the correlation between circulating endotoxins and blood monocyte/macrophage activation in patients with neurodegenerative disease and those endotoxins are found in amyloid plaques in the $\operatorname{AD}$ brain $(89,90)$. Still, the sporadic nature of the LPS-induced $\mathrm{MCl}$ model has led some to question the translational relevance of the model (91). However, the sporadic nature of the model is what allows LPS-induced MCI model to be so useful, as it allows researchers to study the pathogenesis immediately, instead of taking months and even years for a more natural $\mathrm{MCI}$ to develop (92). The LPS model of $\mathrm{MCl}$ also removes aging as an extraneous variable during behavioral assessment, allowing for a more precise study of the effects of neuroinflammation. Despite the sporadic nature of the model, the LPS-induced $\mathrm{MCl}$ model is still susceptible to treatment, where the $\mathrm{MCl}$ can be ameliorated (93). The next session will examine the specific treatment of RT in patients and animals with $\mathrm{MCl}$.

\section{RT can be harnessed to better understand the neuroprotective effects of IGF-1}

Examining $\mathrm{RT}$ as a therapeutic for $\mathrm{MCl}$ in the clinical setting

Strong associations exist between cognition and physical strength in the elderly. For example, muscular weakness measured by grip strength in both women and men who 
were $>65$ years of age had a 1.54 times higher odds ratio of having $\mathrm{MCl}$ after adjustment for age, sex, wealth, years of education, physical activity, obesity, number of chronic physical conditions, and depression (94). In a second study, a randomized double blind, double-sham trial found 18 months of RT significantly improved global cognitive function in patients with $\mathrm{MCl}$ (95), suggesting $\mathrm{RT}$ as a therapeutic to improve outcomes in $\mathrm{MCl}$ patients. Studies examining how to optimize RT programs have focused on the amount of RT workouts per week. One study found biweekly RT for 12-months positively impacted functional plasticity of response inhibition processes in cortex of 65-75-yr old women, while one-weekly resistance-exercise training did not produce the positive impact of RT (96). Conversely, once-weekly or twice-weekly RT has been shown to give cognitive benefits (97). Still, a systematic review of randomized clinical trials reported triweekly RT had a stronger positive effect on cognition compared to biweekly in the elderly (98), suggesting triweekly RT may show some of the strongest effects on cognition.

Optimization of RT programs is not the only part of RT that requires further study, the underlying mechanisms to the improved cognition from $\mathrm{RT}$ in $\mathrm{MCl}$ patients is still largely unknown. Yet, there have been early attempts to narrow it down. A 6-month randomized controlled trial found twice-weekly RT to promote cognition and functional brain plasticity in 70-80 year-old women with $\mathrm{MCl}(99)$. Additionally, studies suggest the effects of RT may be long lasting, as one study found $R T$ to be protective of $A D$-vulnerable hippocampal subfields, protecting those regions from degeneration for at least 12 months after training had ended (100). Another study examining progressive RT found improved cognitive function in patients with $\mathrm{MCl}$ correlated with strength gains and not the improvement in aerobic capacity in these individuals (101). This study suggests optimization of RT programs should focus on strength gains to maximize improvements in cognitive function (101). Even with these clinical studies, RT investigated on a molecular level is necessary 
to better understand the underlying mechanisms behind the ability of RT to improve outcomes in $\mathrm{MCl}$ patients (101).

\section{RT molecular examination}

To better understand RT-induced neuro-adaptations in the brain, animal studies focus on the molecular adaptations of hippocampal regions (CA1, CA3, dentate gyrus) and the prefrontal cortex as these regions play a critical role in memory $(102,103)$. RT has been found to be neuroprotective by reducing neuroinflammation, beta-amyloid, and tauphosphorylation, which are associated with neurodegenerative disease (102). RT has also been shown to be neuroprotective, increasing synaptic plasticity, neurotrophic factors, and neurogenesis, all of which are positively associated with cognition $(102,104,105)$. An example of the benefits of RT have been demonstrated by Liu et al. that found four weeks of RT improved cognition, increased synaptic plasticity markers in the hippocampus and prefrontal cortex, reduced beta-amyloid deposits in the prefrontal cortex, and reduced microglia and astrocyte activation in the hippocampus of 9-month-old transgenic AD mice (102). Although from a glance, it seems the improvement in cognition comes from expected beneficial structural, biochemical, and physiological changes, more mechanistic studies should be performed to determine the underlying molecular mechanisms. As the beneficial responses of RT-induced improvements in cognition are still in question (106). Exercise, in general, has been shown to yield cognitive benefits (107). However, studies molecularly examining both aerobic exercise and RT have found both forms of exercise improve cognition through divergent molecular mechanisms $(108,109)$, suggesting RT should be studied separately. These studies would also suggest that a combination of RT and aerobic exercise could prove synergistic, which is supported from clinical trials in patients with dementia (107). Yet, in rodents, RT has been shown to weaken aerobic exercise-induced cognitive improvements, neurogenesis, and increased 
hippocampal brain-derived neurotrophic (BDNF) (110). Some have suggested RTinduced improvements in cognition to be associated with IGF-1 signaling, while aerobic exercise to be associated with BDNF signaling (109). Conversely, some studies suggest BDNF as a possible reason for the improved cognition from RT as well $(108,111)$. To complicate matters further, IGF-1 has been shown to modulate BDNF (112), bringing into question the specific role of IGF-1 in RT-induced benefits in cognition. Additionally, there is a discrepancy in the literature on whether RT can induce neurogenesis $(105,113)$, which is largely associated with BDNF and IGF-1 signaling as well as exercise-induced benefits in cognition (114-116). Taken together, the current literature has left unanswered questions regarding the underlying molecular signaling involved in RT-induced cognitive benefits, specifically referring the neurotrophic response of IGF-1 signaling (101). Hence, the primary focus of my dissertation was to uncover the role of IGF-1 signaling as it pertains to RT and cognition. The next section will review the role of IGF-1 as it relates to $\mathrm{MCl}$.

\section{The essential role of IGF-1 signaling in the neurodegeneration process}

The neuroprotective attributes of IGF-1 combined with IGF-1 dysregulation observed in patients with neurodegenerative disease has led to the hypothesis that the loss IGF-1 input contributes to the neurodegenerative process (117-119). The neuroprotective effects of IGF-1 could include reducing neuroinflammation, reducing neural excitotoxicity, enhancing cell proliferation, and enhancing neural cell survival $(120,121)$. Although many studies show IGF-1 to have anti-inflammatory effects $(120,122,123)$, the IGF-1 effects on neuroinflammation remain controversial. For example, IGF-1 and IGF-1 gene transfer have both been shown to inhibit neuroinflammation, where IGF-1 signaling inhibition resulted in reduced neuroinflammation in $\operatorname{AD}$ mice $(124,125)$. One potential explanation for the discrepancy of IGF-1 effects on neuroinflammation may be related the amount of 
IGF-1 signaling that occurs as IGF-1 can either protect against or increase LPS-induced damage depending on dosage of IGF-1 (126). IGF-1 can also be neuroprotective by reducing neural excitotoxicity. IGF-1 alleviates excitotoxicity in cultured hippocampal neurons mediated by IGF-1 inhibition on glutamate receptors through PI3K-AKT-mTOR signaling (127). IGF-1 may also exert protection against excitotoxicity through astrocytic regulation (121). Chen et al. found astrocytic IGF-1 gene transfer decreased tau phosphorylation and improved cognition in a mouse model of neurodegeneration (121). In addition, IGF-1 is thought to exert effects of neuroprotection through enhanced cell proliferation and cell survival (115). Cells treated with IGF-1 yield the highest cell proliferation and survival compared to other growth media (115). Collectively, these data suggest IGF-1 signaling can employ a wide range of neuroprotective benefits, which could be useful in ameliorating neurodegeneration and cognition. 


\section{Chapter Two: Resistance-exercise training ameliorates LPS-induced cognitive impairment concurrent with molecular signaling changes in the rat dentate gyrus}

Taylor Kelty ${ }^{1}$, Todd R. Schachtman ${ }^{4}$, Xuansong Mao ${ }^{1}$, Kolter B. Grigsby ${ }^{1}$, Thomas E. Childs $^{1}$, T. Dylan Olver ${ }^{6}$, Paige N. Michener ${ }^{4}$, Rachel A. Richardson ${ }^{4}$, Christian K. Roberts $^{7}$, and Frank W. Booth ${ }^{1,2,3,5}$

${ }^{1}$ Department of Biomedical Sciences

${ }^{2}$ Department of Nutrition and Exercise Physiology

${ }^{3}$ Department of Pharmacology and Physiology

${ }^{4}$ Department of Psychology

${ }^{5}$ Dalton Cardiovascular Center

University of Missouri

Columbia, Missouri 65211, U.S.A.

${ }^{6}$ Department of Biomedical Sciences,

Western College of Veterinary Medicine,

University of Saskatchewan,

Saskatoon, Saskatchewan, Canada, S7N 5B4

${ }^{7}$ Geriatrics, Research, Education and Clinical Center

VA of Greater Los Angeles Healthcare System

Los Angeles, California, USA 


\section{Abstract}

Effective treatments preventing brain neuroinflammatory diseases are lacking. Resistance-exercise training (RT) ameliorates mild cognitive impairment (MCl), a forerunner to neuroinflammatory diseases. However, few studies have addressed the molecular basis by which RT abates $\mathrm{MCl}$. Thus, experiments were performed to identify some molecular changes occurring in response to RT in young, female Wistar rats. To induce $\mathrm{MCl}$, intraventricular lipopolysaccharide injections were used to increase dentate gyrus inflammation, reflected by significantly increased TNFa ( 400\%) and IL-1 $\beta$ $(\sim 1,500 \%)$ mRNA $(p \leq 0.001)$ after 6-weeks. Five days after LPS injections, half of LPS injected rats either performed three days/week of RT by ladder climbing for 6-weeks, while half remained without ladders. RT for 6 -weeks increased lean body mass percentage ( $p$ $\leq 0.05$ ), individual muscle masses (gastrocnemius and tibialis anterior) ( $p \leq 0.05$ ), and maximum lifting capacity ( $p \leq 0.001)$. The RT group, compared to sedentary controls, had: 1) ameliorated spatial learning functions ( $p \leq 0.05)$, 2) increased dentate gyrus phosphorylation of IGF-1R, AKT and GSK3 proteins ( $p \leq 0.05$ ), components of downstream IGF-I signaling, and 3) increased dentate gyrus synaptic-plasticity-marker SYN1 protein ( $p \leq 0.05)$. Two follow-up experiments (without LPS) characterized dentate gyrus signaling during short-term RT. Twenty-four hours following the third workout in a 1-week training duration, phosphorylation of ERK1/2 and GSK3 $\beta$ proteins, as well as proliferation-marker protein, PCNA, were significantly increased ( $p \leq 0.05)$. Similar changes did not occur in a separate group of rats following a single RT workout. Taken together, these data indicate that RT ameliorates LPS-induced $\mathrm{MCI}$, possibly mediated by increased IGF-1 signaling pathway components within the dentate gyrus. 


\section{Introduction}

Neurodegenerative disease prevalence is increasing, as the worldwide population becomes older (128). Neuroinflammation is thought to be a part of this neurodegenerative process (129). Lipopolysaccharide (LPS)-induced neuroinflammation is a model of neurodegenerative diseases, such as Parkinson's disease, multiple sclerosis, and $\mathrm{AD}(91)$. In each of these diseases, cognitive deficits are observed $(128,130,131)$. However, their disease mechanisms are poorly understood and effective therapies are lacking (132), making it important to explore all potential avenues of therapy that could prevent or delay the progression of these diseases.

Emerging evidence suggests that resistance-exercise training (RT) can improve cognition $(97,99,133)$. Singh et al. (95) found that 6 -months (2-3 days/week) of RT improved cognition in older adults with MCl. Additionally, Marvos et al. (101) had older adults with $\mathrm{MCl}$ undergo six months of RT (2-3 days/week) and found improvements in strength and cognition, and concluded that the strength gains mediated the cognitive benefits. However, the molecular signaling leading to cognitive benefits in patients with $\mathrm{MCl}$ is still largely unknown. Marston et al. (134) supports this, as they suggested that $\mathrm{RT}$ is an understudied therapy to potentially improve cognitive health.

Studies examining RT in models without cognitive deficits have noted that RT leads to increases in IGF-1 signaling and markers of neuroplasticity within the brain (104, 109, 135). IGF-1 signaling activates the conical AKT and ERK1/2 pathways leading to greater neuronal proliferation, survival, and plasticity $(136,137)$. In addition, IGF-1 overexpression has been shown to improve spatial learning memory and neurogenesis in older mice (138), proposing IGF-1 signaling as a candidate pathway for the improvement in memory induced by RT (104). One earlier study examined the effects of RT in the hippocampus of epileptic rats and found $\mathrm{RT}$ ameliorated $\mathrm{MCl}$ associated with BDNF protein signaling 
(139). To our knowledge, no other study has examined molecular signaling induced by RT in rodents with $\mathrm{MCl}$.

Therefore, to better understand RT effects on $\mathrm{MCl}$, we studied IGF-1 signaling in the dentate gyrus associated with RT in a lipopolysaccharide (LPS)-injected model of $\mathrm{MCl}$. The studies had an additional goal of establishing in the Wistar rat a RT protocol that more closely resembles human RT. We used female rats that underwent 6-weeks of RT by climbing a ladder with a progressive loading protocol of increasing weights attached to their tail. To test for spatial memory differences, rats underwent cognitive behavioral testing using the Barnes maze. After rats were euthanized, the dentate gyrus was removed for molecular analysis of IGF-1 signaling and markers of neuroplasticity and proliferation. We hypothesized that 6-weeks of RT would 1) ameliorate cognitive functions induced by LPS; 2) increase IGF-1 signaling and markers of neuroplasticity and proliferation in the dentate gyrus; 3) and induce muscle hypertrophy and increase lean mass. Additionally, in a second study without LPS, rats underwent one workout or three RT workouts (over a single week) were euthanized 24-hrs after their last workout to investigate the effects of short-term RT on IGF-1 signaling.

\section{Materials and Methods}

Animals and exercise protocols

Female, Wistar rats were bred at University of Missouri where, upon weaning, were group housed until surgery. Rats were maintained on a $12: 12$-hr light/dark cycle at $24^{\circ} \mathrm{C}$ and were provided food and water (Formulab Diet 5008, Purina) ad libitum during the entire study. The University of Missouri Animal Care and Use Committee approved experimental

protocols. For Experiment 1, five week-old rats were divided into three groups: Vehicle (VEH) injected, LPS-injected, and LPS injected with 6-weeks RT (LPS + RT) ( $n=10-14$ 
rats/group). Experiment two examined the effects of $\mathrm{RT}$ in the dentate gyrus such that five week-old rats (without LPS) were divided into sedentary (SED) and resistance-exercise trained $(\mathrm{RT})$ groups (that underwent either one or three RT workouts). All groups were pair-matched based on bodyweight ( $\mathrm{n}=$ seven per group).

\section{Induction of brain inflammation}

To induce brain inflammation, rats underwent intracerebroventricular injections of LPS (Milliporesigma, St. Louis, MO), which was adapted from our lab's published protocol of nucleus accumbens-specific injection (140). Rats were anesthetized and positioned into a stereotaxic frame (David Kopf Instruments, Tunjunga, CA). Using a 25 ul Hamilton syringe (Hamilton Co., Reno, NV) LPS (4.54 ug/ul) or the vehicle (artificial cerebrospinal fluid) were injected into the skull at $0.8-\mathrm{mm}$ posterior to bregma,1.5-mm lateral to the midline suture, and 3.6-mm below the skull surface at a rate of $1 \mathrm{ul} / \mathrm{minute}$ for a total of five mins, similarly to other published work to induce brain inflammation $(141,142)$. Rats were then given five days to recover (singly housed), before the exercise protocol began (group housed for the remainder of the study).

\section{Exercise protocols}

All animals were subjected to two days of familiarization to the ladder apparatus (Fig. 2.1). Each familiarization day included a single set of climbing where each animal started at the bottom of the ladder and climbed 100-cm into the housing chamber (Fig. 2.1) at the top of the ladder. It should be noted that the rats did not require electric shock to climb the ladder either in the 2-day familiarization period, or during the subsequent RT workouts. All rats received a sugar treat the first week, regardless of the group they were in, as a positive reinforcer. Also, when rats did not want to climb (i.e., during their maximum loads), the investigator assisted by lifting the weight attached to the tail to help them initiate the first 
step on the ladder and then gently let go of the weight as they voluntarily proceeded to the second step, at which point the rats climbed voluntarily to the top of the ladder.

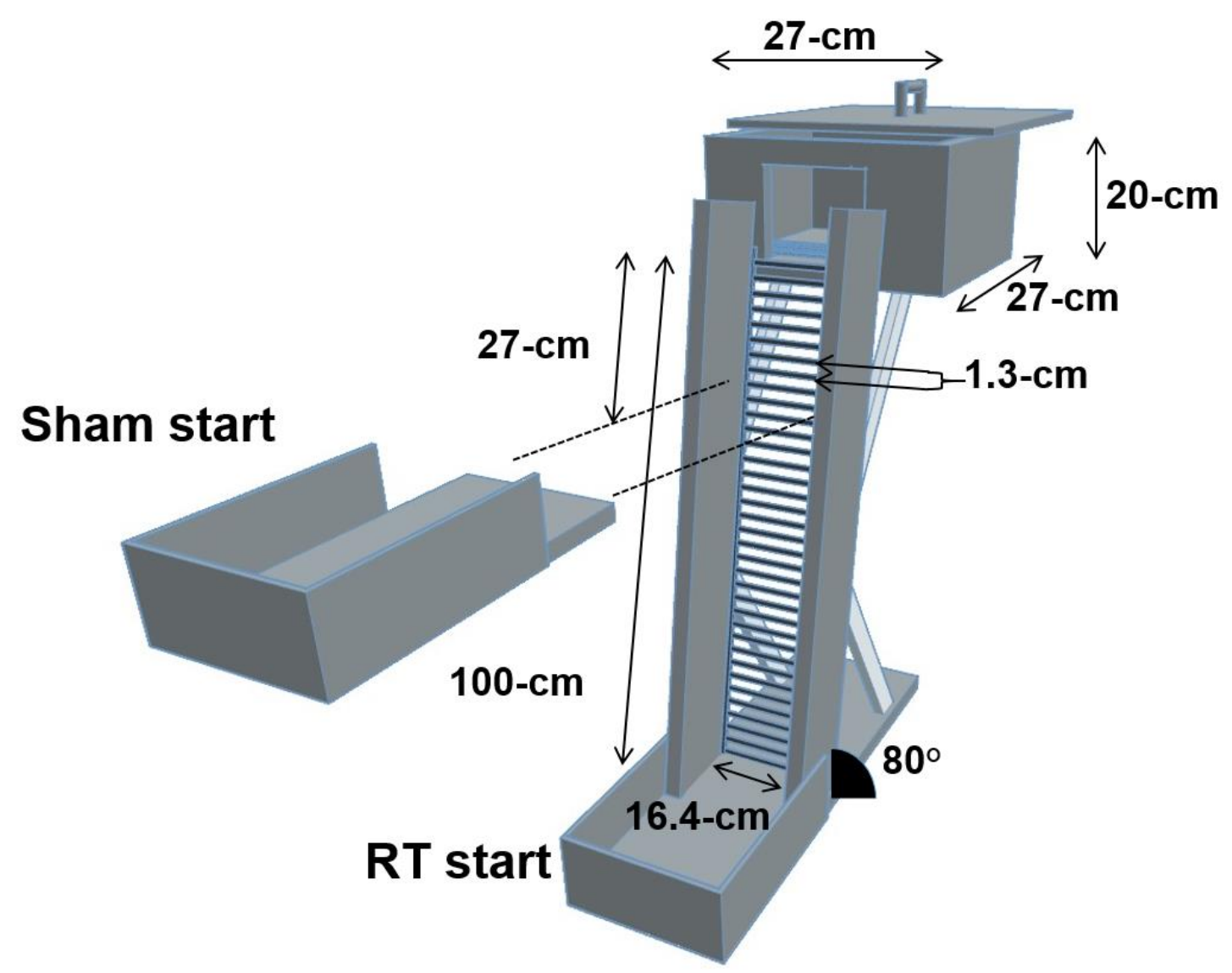

Figure 2.1. Schematic of ladder climbing apparatus. 
Experiment one - Rats trained 3x/week for 6-weeks with weights placed in a bag that was attached to the base of the tail with athletic tape (3M, Maplewood, MN). The first workout of each week was a maximum-lifting day to determine each rat's maximum lifting capability. Each workout started with a warm-up set where the rat was allowed to climb freely (without weight) into the housing chamber and then rest for 1-minute. Following the warm-up, each maximum-lifting day consisted of eight sets (with 1-minute rest periods between), where a set is defined as the animal completing a single 100-cm climb. In weeks $2-6$, the first of eight sets started at $50 \%$ of their previous week's maximum (for the first week the first set was $50 \%$ of that individual rat's body weight as the maximum lifting capability was unknown in week one). Weights then were progressively increased by 2550 grams per set until the rat was unable to climb and reach the housing chamber (allowing weekly progressive increase in loading to be individualized to each rat's performance). The remaining two days of workouts during the week also consisted of eight sets (after the warm up) at $50,50,75,75,90,100,100$, and $100 \%$ (from set one to eight, respectively) of the rat's maximum determined on day one of that week. The exercise sham groups (LPS and VEH) climbed the ladder without tail weights three days/week, but climbed 27$\mathrm{cm}$ once (instead of 100-cm repeated seven more times) (Fig. 2.1), with a 1-minute rest period in the housing chamber, then were placed back into their home cage. This ladder climbing protocol was adapted from previously published studies $(109,143)$.

Experiment two - Rats performed a single workout of eight sets (1 workout), or three training workouts ( 1 workout every other day for one week). Rats that underwent three days of training had workouts that were similar to Experiment 1, where maximum lifting capability was determined on the first workout of the week, and the subsequent two workout days consisted of eight sets (after the warm up) at 50, 50, 75, 75, 90, 100, 100, 
and $100 \%$ of the individual rats maximum. SED group was given sham-RT similar to Experiment 1's sham groups.

\section{Barnes maze testing}

To test spatial memory (during the $5^{\text {th }}$ week of training), a gray, Barnes maze circular platform (122-cm in diameter), with 20 holes (each being 10.2-cm in diameter) was used. The circular platform had 30.5-cm walls where visual cues (white, cut out, cardboard shapes) hung to aid in the rats search for the target hole in which an escape box (28-cm in length $\times 12.7-\mathrm{cm}$ wide $\times 7.6-\mathrm{cm}$ high), which was located below the hole such that the rat could climb down into. Two 86 -Watt, 120-Volt floodlights producing 1,690 Im hung above the platform to create an aversive environment motivating the rat to locate and enter the dark escape box. A black curtain was hung to create a spotlight effect on the Barnes maze, amplifying the aversive environment and increasing motivation for the rats to enter the escape box. Each rat was assigned a hole where the target escape box was located (assigned holes varied among rats to eliminate odor cues when testing). The hole location of the escape box remained constant for each individual rat throughout the entirety of testing. Testing took place during the light cycle between 10:00 am and 1:00 pm and consisted of two trials a day (1 hour apart) for four consecutive days (day one only had one trial): a familiarization phase (day 1), a learning phase (days 2-3), and a testing phase (day 4). The first day included only one trial (familiarization trial) where each rat was acclimated to the escape box for one minute, and then immediately placed in the center of the Barnes maze platform under a start box. The rat sat under the start box for 30 seconds, and then was allowed to explore the maze freely for a maximum of five minutes. The trial ended when the rat entered the escape box on its own or the five-minute trial ended. If the rat did not find the escape box at the end of the five minutes, it was gently guided to the escape box. The rat then sat in the escape box for 30 seconds to help 
associate the escape box with safety. Following the 30 seconds in the escape box, the experimenter gently placed the rat back into its home cage, and the Barnes maze was cleaned with $70 \%$ ethanol. The following trials (learning and testing phase on days $2-4$ ) were repeated in the same manner, with the exception of the rat not being acclimated to the escape box at the beginning of the trial (due to the rat already being familiar with the escape box). The time taken to locate the escape box (place front paws into the box) and the number of errors (nose pokes into holes that do not contain the escape box) was recorded. Design and methods used were adapted from Olver et al. (144).

\section{Dual-energy X-ray absorptiometry (DEXA)}

Following euthanasia by $\mathrm{CO}_{2}$ asphyxiation, rat's lean muscle mass percentages were measured by DEXA (QDR 4500A, Hologic, Inc., Bedford, MA), calibrated for rats.

\section{Tissue collection}

Following euthanasia by $\mathrm{CO}_{2}$ asphyxiation, 24-hrs after the final ladder-climbing or sham workout groups, dentate gyrus punches of 3-mm in diameter were taken from 2-mm thick coronal brain slices sliced using a brain matrix (Braintree Scientific Inc., Braintree, MA), and identified using a rat brain atlas (Paxinos and Watson, 1997). Dentate gyrus punches were flash frozen with liquid nitrogen and stored at $-80^{\circ} \mathrm{C}$ until processing. Skeletal muscles were extracted and weighed on a precision balance (Mettler Toledo Inc., Columbus, $\mathrm{OH}$ ) to obtain wet weight of each muscle.

\section{RNA isolation, cDNA synthesis, and $q R T-P C R$}

Following six weeks of RT, or sham-training rats, were euthanized and the dentate gyrus was removed as described above. Dentate gyrus mRNA isolation, cDNA synthesis, and qRT-PCR were performed as previously described (140). Briefly, dentate gyrus punches 
were placed in TRIzol (Invitrogen, Carlsbad, CA) and homogenized 3X for one minute at $25 \mathrm{~Hz}$ using RNAse-free, stainless steel beads and a Tissuelyser (Qiagen, Germantown, MD). To prevent degradation of samples, the samples were set on ice for 1-minute between each 1-minute homogenizations. Using the TRIzol protocol, RNA was extracted and then quantified using Nanodrop 1000 (Thermo Scientific, Waltham, MA). For cDNA synthesis, RNA was treated with DNAase using DNase I (Thermo Scientific, Waltham, MA) and reverse transcribed using a High Capacity cDNA Reverse Transcription Kit (Applied Biosystems, Carlsbad, CA). For qRT-PCR, gene-specific primers were constructed using Primer3 (Table 2.1). cDNA from each sample were assayed in duplicates for the target genes in Table 2.1 using iTaq Universal SYBR green Supermix (Bio-Rad Laboratories, Hercules, CA). mRNA expression values were quantified by the $2^{\Delta \Lambda C t}$ method normalized to $18 \mathrm{~S}$. 


\begin{tabular}{|c|c|c|c|}
\hline Gene & Forward $\left(5^{\prime}-3^{\prime}\right)$ & Reverse $\left(5^{\prime}-3^{\prime}\right)$ & Accession number \\
\hline $18 \mathrm{~S}$ & GCCGCTAGAGGTGAAATTCTTG & CATTCTTGGCAAATGCTTTCG & NR_046237 \\
\hline TNF- $a$ & AACACACGAGACGCTGAAGT & TCCAGTGAGTTCCGAAAGCC & NM_012675 \\
\hline IL-6 & CAGTTGCCTTCTTGGGACTGA & GAAGTCTCCTCTCCGGACTTG & NM_012589 \\
\hline $\mathrm{IL}-1 \beta$ & TGACTTCACCATGGAACCCG & GACCTGACTTGGCAGAGGAC & NM_031512 \\
\hline
\end{tabular}

Table 2.1. Primers used for $q R T-P C R$. 


\section{Western blotting}

Following dentate gyrus tissue collection, the tissue was homogenized in RIPA buffer (50 mM Tris-HCL (pH 8.0), $150 \mathrm{mM} \mathrm{NaCl}, 1 \% \mathrm{NP}-40,0.5 \%$ sodium deoxycholate, $1 \%$ SDS, 1x protease inhibitor cocktail) using stainless-steel beads and a Tissuelyser (Qiagen, Valencia, CA) $3 \mathrm{X}$ at $25 \mathrm{~Hz}$ for one minute. To prevent degradation of samples, the samples were set on ice for one minute between each homogenization similar to methods described above for mRNA. Homogenized samples then underwent centrifugation at $12,000 \mathrm{~g}$ for ten minutes with the supernatant protein concentrations obtained using the BCA assay (Pierce Biotechnology, Rockford, IL). Forty micrograms of protein in loading buffer was loaded onto $10 \%$ SDS-PAGE gels. Proteins were then transferred to nitrocellulose membranes and all blots were incubated with Ponceau S (Milliporesigma, St. Louis, MO) to verify equal loading in each lane. After using $5 \%$ milk in Tris-buffered saline + Tween20 (TBS-T) to block non-specific binding, primary antibodies (rabbit polycolonal) insulin-like growth factor one beta receptor (IGF-1R 3 ) (95kDa), p44/42 extracellular signal-regulated kinases (ERK1/2) (42, 44 kDa), glycogen synthase kinase three beta (GSK-3 $\beta$ ) (46 kDa), phospho-protein kinase B (p-AKT) (Ser473) (60 kDa), phospho-p44/42 extracellular signal-regulated kinases (p-ERK1/2) $(42,44$ kDa), phosphoglycogen synthase kinase three beta (p-GSK-3ß) (Ser9) (46 kDa), synapsin (SYN1)(77 kDa), and proliferating cell nuclear antigen (PCNA)(36 kDA) 1:1000 (Cell Signaling, Inc., Danvers, MA) diluted in TBS-T with 5\% bovine serum albumin were applied overnight at $4^{\circ} \mathrm{C}$. Primary antibodies protein kinase $\mathrm{B}(\mathrm{AKT})(60 \mathrm{kDa})$ at $1: 2000$ and glyceraldehyde 3phosphate dehydrogenase (GAPDH) (37kDa) (Cell Signaling, Inc., Danvers, MA) at 1:20,000 were applied overnight at $4^{\circ} \mathrm{C}$. In addition to Ponceau S, GAPDH was also used to verify equal loading. Additionally, 40 micrograms of protein was probed for phospho- 
insulin-like growth factor one beta-receptor (p-IGF-1Rß) (y1135/1136) (Cell Signaling, Inc., Danvers, MA) at 1:375 diluted in TBS-T with $5 \%$ bovine serum albumin, which was applied for three days at $4^{\circ} \mathrm{C}$. Next, horseradish peroxidase-conjugated secondary antibody at a dilution of 1:1000 (Cell Signaling Technology, Inc., Danvers, MA) was applied for one hour at room temperature and Immobilon Forte Western HRP substrate (Milliporesigma, St. Louis, MO) was applied for five minutes prior to exposure. Molecular weight was estimated using a PageRuler Prestained Protein Ladder (ThermoFischer, Waltham, MA). The Kodak 4000R Imager and Molecular Imagery Software (Kodak Molecular Imaging Systems, New Haven, CT) were used to determine band densitometry.

\section{Statistical analyses}

All analytical procedures were evaluated using SigmaPlot 14.0 (Systat Software Inc., San Jose, CA). T-tests were performed for all comparisons between SED and RT groups. All other comparisons were performed using one-way ANOVA followed by Holm-Sidak posthoc. A $p \leq 0.05$ was the significance level. All values are expressed as mean and standard error of mean.

\section{Results}

\section{Experiment 1}

To verify LPS injections, qRT-PCR was used in three experimental groups to measure known markers of inflammation stimulated by LPS. For TNFa mRNA, VEH vs LPS was $1.00 \pm 0.13$ vs $3.58 \pm 0.60$ fold increase, respectively; and VEH vs LPS + RT was $1.00 \pm$ 0.13 vs $3.61 \pm 0.95$ fold increase, respectively, with $p \leq 0.001$ for both comparisons. For IL-1 $\beta$ mRNA, VEH vs LPS was $1.00 \pm 0.23$ vs $18.13 \pm 2.23$ fold increase, respectively;

and VEH vs LPS + RT was $1.00 \pm 0.23$ vs $17.14 \pm 5.27$ fold increase, respectively, with $p$ $\leq 0.001$ for both treatment groups from VEH. There was no change in IL-6 mRNA between 
groups (Fig. 2.2). In summary, both TNFa mRNA and IL-1 $\beta$ mRNA were significantly increased in the dentate gyrus of LPS and LPS + RT groups compared to VEH, suggesting LPS injections were sufficient to induce neuroinflammation in the dentate gyrus. 


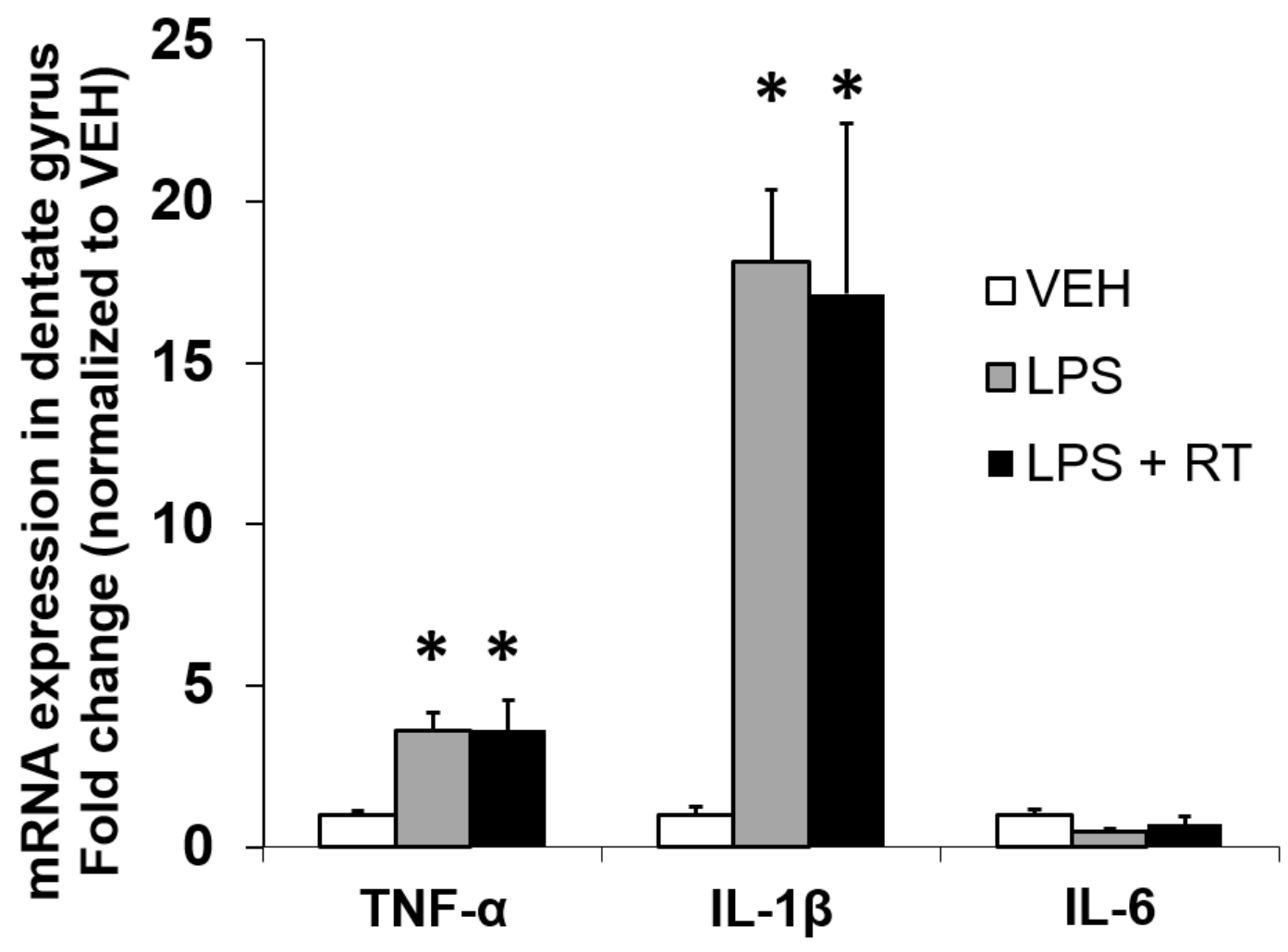

Figure 2.2. Proinflammatory marker levels in VEH, LPS, and LPS + RT groups following six weeks of RT or sham training. ${ }^{*}=p \leq 0.001$ significantly different from VEH group. One-way ANOVA, Holm-Sidak post-hoc test, $\mathrm{n}=7-10$ per group. 
The efficacy of the 6-weeks RT was established by the next determinations. The LPS + RT group significantly increased their maximum weight (shown in grams) lifted by the third week of training, week one vs week three was $147 \pm$ four vs $222 \pm 11$, respectively, with $p \leq 0.001$ (Fig. 2.3). The LPS + RT group, compared to the LPS group, showed a significant increased in lean muscle mass percentage, LPS vs LPS + RT was $88.25 \pm 0.72$ vs $91.15 \pm 0.29$, respectively, with $p \leq 0.05$ (Fig. 2.4A). Compared to VEH or LPS groups, the LPS + RT group showed a significant increase in the gastrocnemius muscle wet weight, VEH vs LPS + RT was $5.315 \pm 0.17$ vs $5.831 \pm 0.20$, and LPS vs LPS + RT was $5.317 \pm 0.09$ vs $5.831 \pm 0.20$, respectively, both with $p \leq 0.01$ (Fig. $2.4 \mathrm{C}$ ). Additionally LPS + RT group showed a significant increase in the tibialis anterior wet weight, VEH vs LPS + RT was $2.170 \pm 0.10$ vs $2.491 \pm 0.08$, and LPS vs LPS + RT was $2.287 \pm 0.06$ vs 2.491 \pm 0.08 , respectively, both with $p \leq 0.05$ (Fig. 2.4D). Compared to VEH, LPS + RT showed a significant increase in extensor digitorum longus muscle wet weight, VEH vs LPS + RT, $0.473 \pm 0.02$ vs $0.523 \pm 0.02$, respectively, with $p \leq 0.05$ (Fig. 2.4E). No significant changes by treatments were noted in the wet weight of plantaris muscle in the hind limb (Fig. 2.4F). No differences in body weight were observed over the course of the study among the groups (Fig. 2.4B). In summary, these increases in strength and percentage lean mass are similar to those noted with strength training in humans, suggesting ladder climbing by rats, as employed here, could be a translatable model of $\mathrm{RT}(8,9)$. 


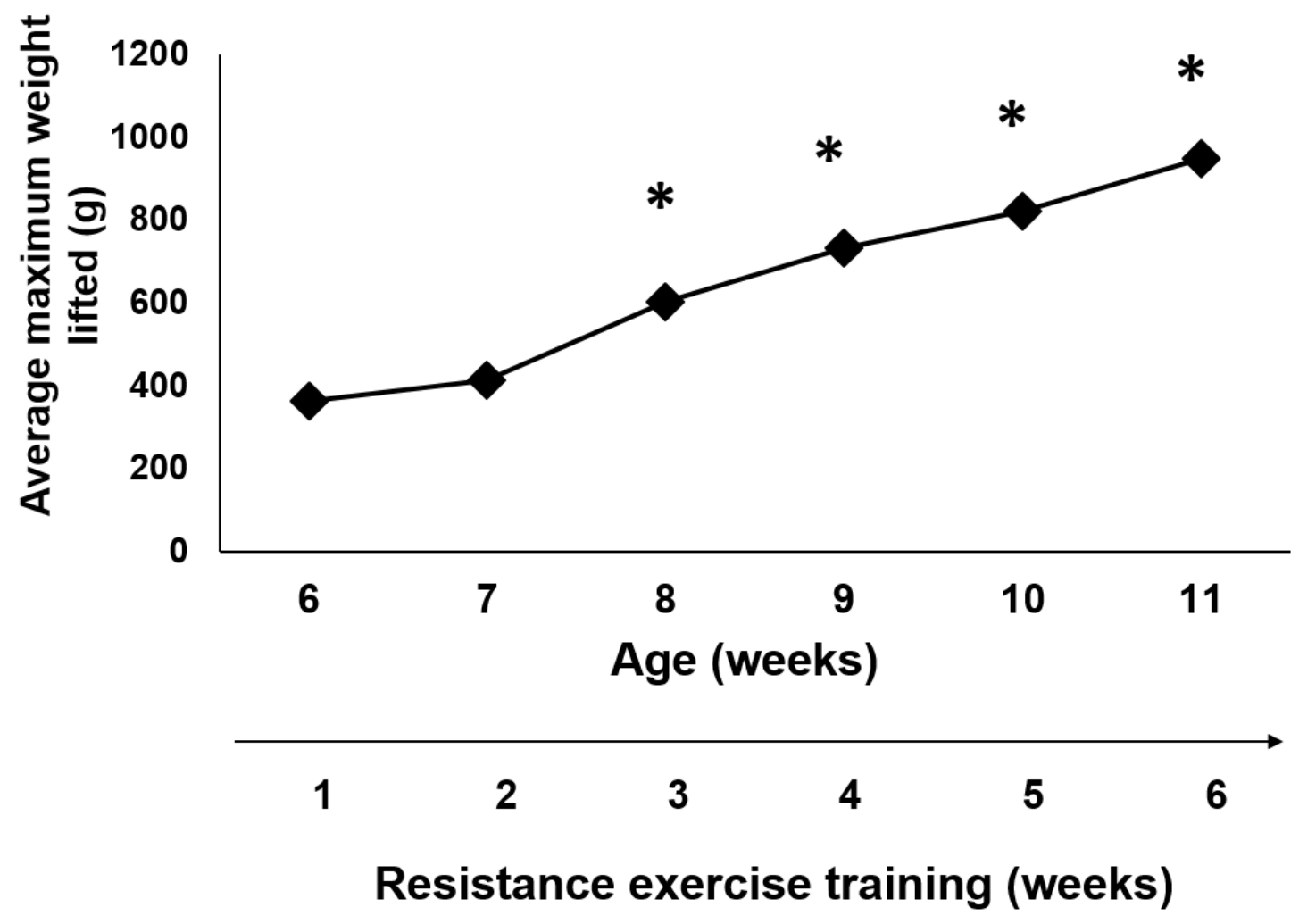

Figure 2.3. Maximum weight lifted weekly during 6-weeks of progressive RT in rats with LPS

(LPS + RT). Maximum weight lifted is presented as peak weight lifted (weight attached to tail + body weight) during first RT workout each week. ${ }^{*}=p \leq 0.001$ significantly different from week 1. One-way ANOVA, Holm-Sidak post-hoc test, $\mathrm{n}=10$. 


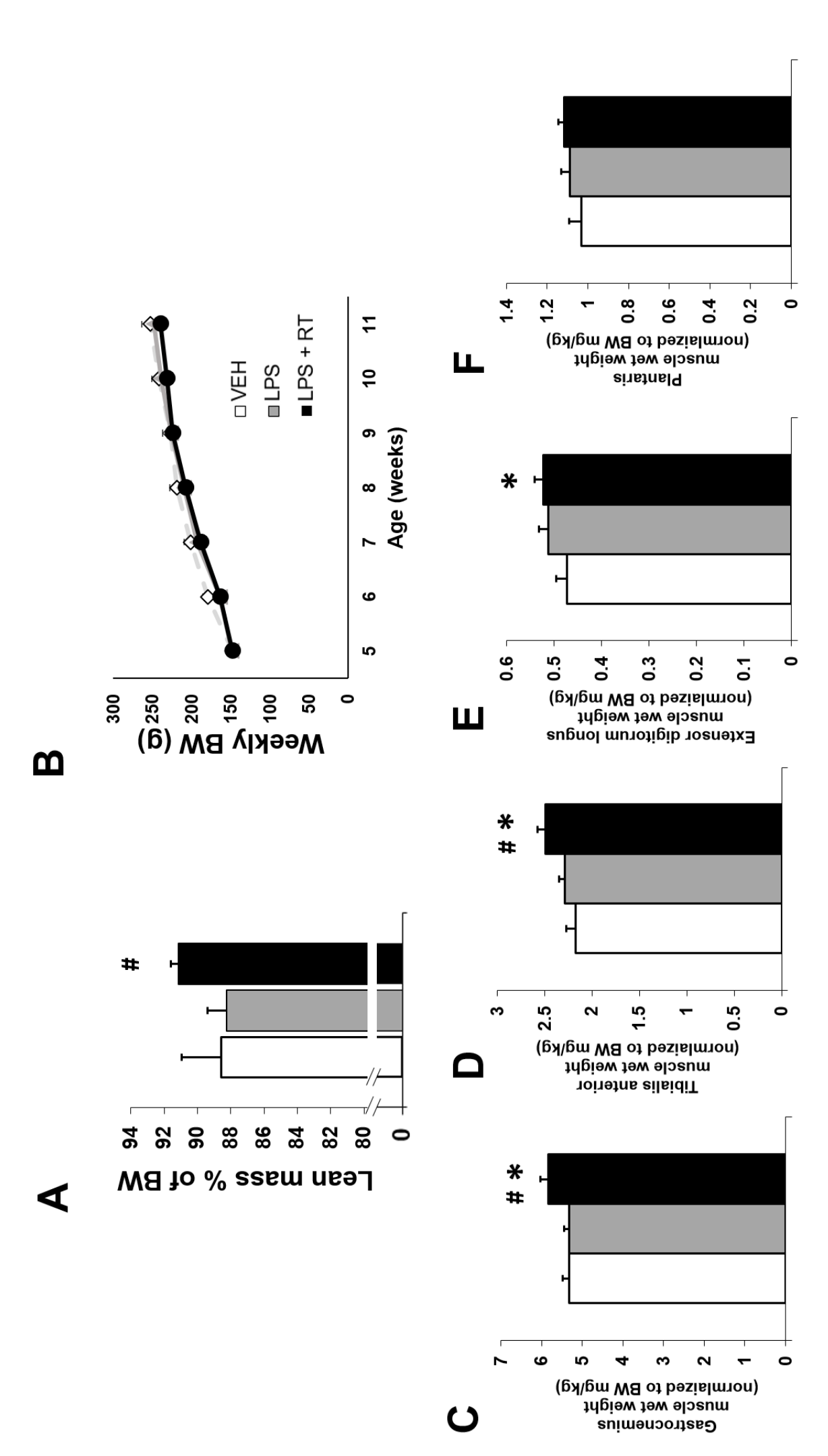


Figure 2.4. Effects of LPS without exercise (LPS group), or LPS with 6-weeks of RT (LPS + RT group) on lean mass, body weight, and skeletal muscle masses. A) lean mass, as a percentage of total body weight. B) Body weight (BW) over 6-weeks of RT. C-F) Muscle wet weights are shown as the fraction of $\mathrm{mg}$ muscle wet weight/ $\mathrm{kg}$ body weight ${ }^{*}=\mathrm{p} \leq$ 0.05 significantly different from VEH group. $\#=p \leq 0.05$ significantly different from LPS group. One-way ANOVA, Holm-Sidak post-hoc test, per group, with $n=$ nine - ten per group. 
Throughout testing each group's average latency (in seconds) progressively decreased through day four ( $p \leq 0.05$, Fig. $2.5 \mathrm{~A})$, indicating spatial learning in all groups. Concurring with our hypothesis, LPS + RT had reduced average latencies on day four (test day), LPS vs LPS + RT, $118 \pm 24$ vs $52.3 \pm 13$, respectively, with $p \leq 0.05$ (Fig. $2.5 \mathrm{~A}$ ), compared with the LPS group. The average number of errors made were significantly decreased between days one and four in only the LPS + RT, day one vs day four was $10.3 \pm 2.4$ vs $3.78 \pm 1.2$, respectively, with $p \leq 0.05$ (Fig. 2.5B). There were no other reported differences in Barnes maze testing. Taken together, these data suggest RT was able to reverse spatial learning deficits induced by LPS using the procedure and parameters of the present experiments. 

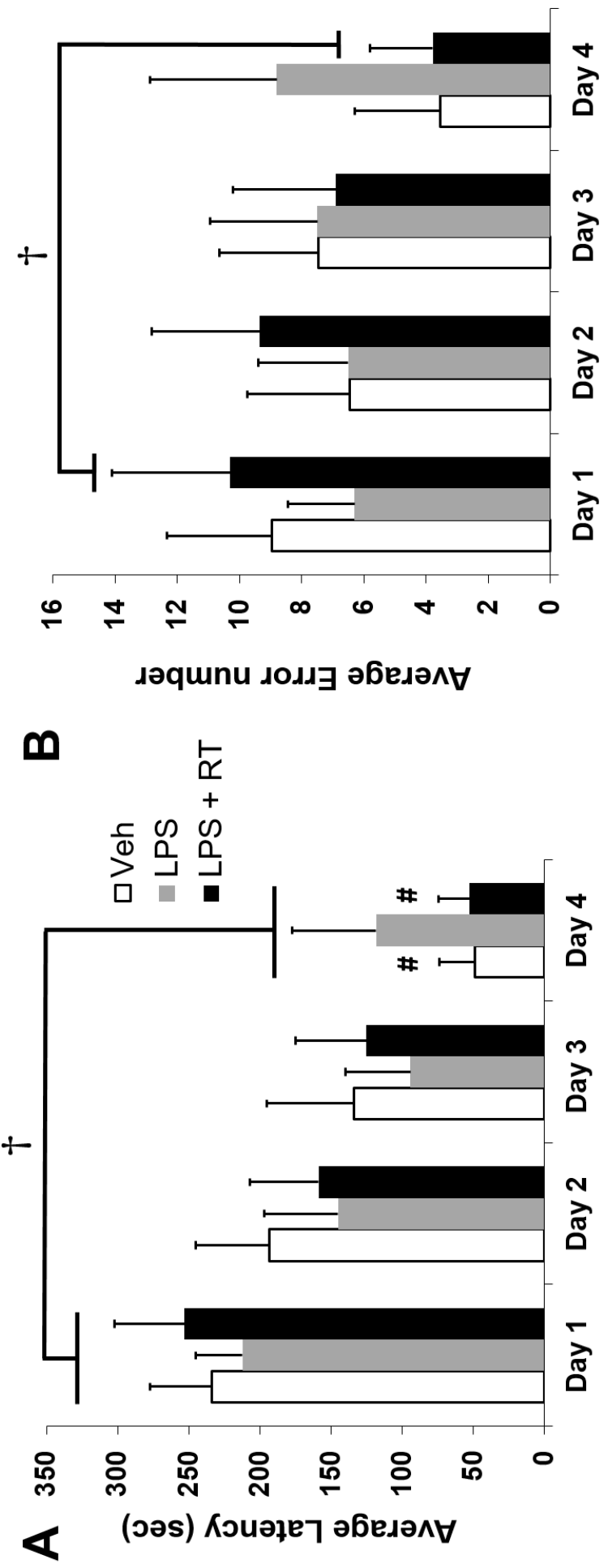
Figure 2.5. Effects of RT on spatial learning and Barnes maze errors five weeks into RT or sham-training. A) Latency to locate goal box. B) Errors made to locate goal box. \# = p $\leq 0.05$ significantly different from LPS group. $\dagger=p \leq .0 .05$ significantly different between day one and day 4. One-way ANOVA, Holm-Sidak post-hoc test, $n=9-14$ per group. 
To determine molecular signaling, the dentate gyrus was collected from all groups at the end of the 6-weeks to test the hypothesis that 6-week resistance-exercise training would increase downstream IGF-I signaling. Compared to LPS group, the LPS + RT group had increased: a) IGF-1R phosphorylation, LPS vs LPS + RT was $0.75 \pm 0.126$ vs $1.15 \pm 0.07$ fold increase, b) AKT phosphorylation, LPS vs LPS + RT was $0.93 \pm 0.08$ vs $1.65 \pm 0.29$ fold increase, c) GSK3 $\beta$ phosphorylation, LPS vs LPS + RT was $1.06 \pm 0.32$ vs $2.48 \pm$ 0.54 fold increase, and d) SYN1 protein, LPS vs LPS + RT was $0.63 \pm 0.10$ vs $1.74 \pm 0.46$ fold increase, all comparisons had $p \leq 0.05$ (Fig. 2.6A \& B). However, no changes in ERK1/2 phosphorylation (Fig. 2.6A) or PCNA protein concentration (Fig. 2.6B) were observed. Compared to the LPS group, total IGF-1R protein expression was significantly higher in LPS + RT group, LPS vs LPS + RT was $0.89 \pm 0.09$. vs $1.22 \pm 0.09$ fold increase. No changes in other total proteins, nor $\mathrm{p}-\mathrm{ERK} 1 / 2$, was observed. Taken together, the hypothesis was held as selected components of downstream IGF-1 signaling were increased by RT. 


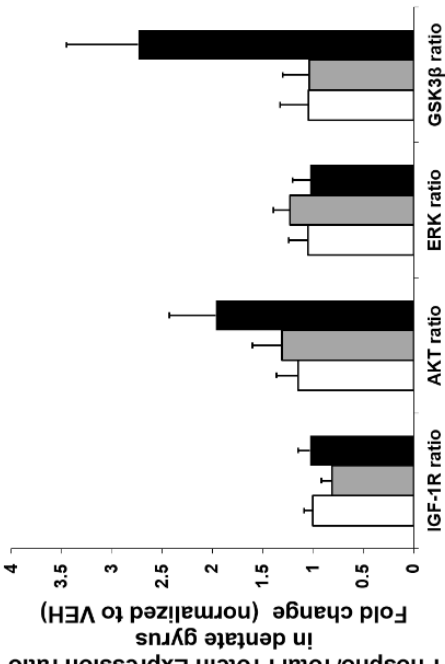

0

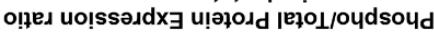

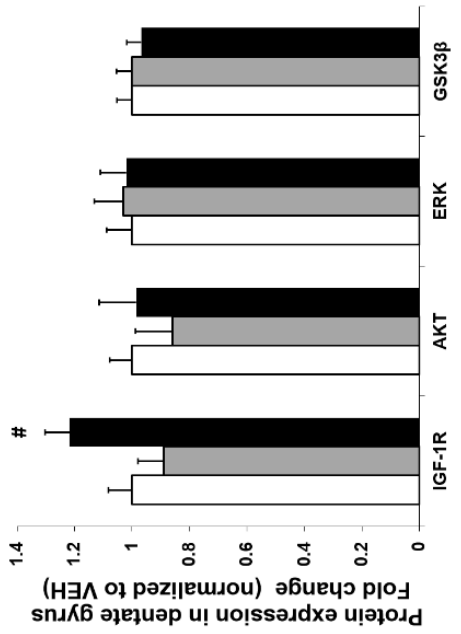

$\boldsymbol{m}$

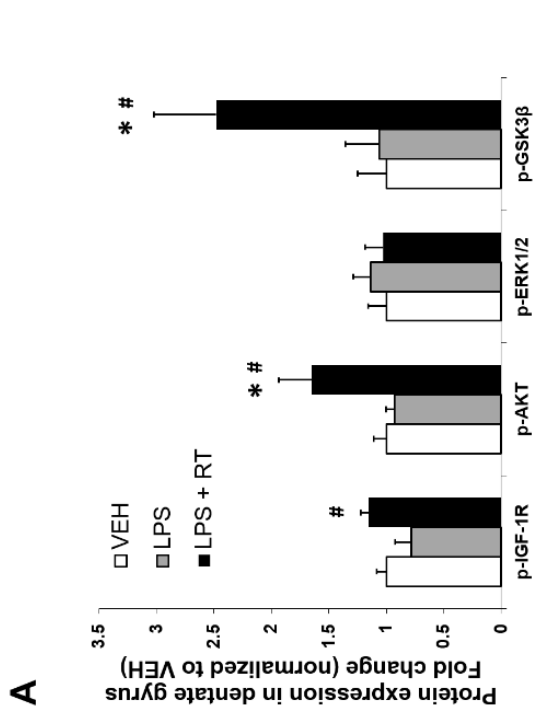

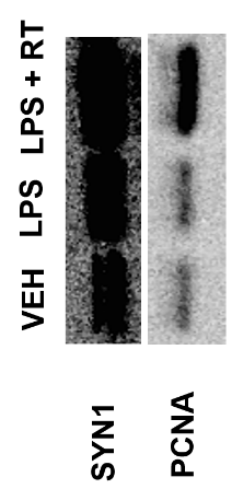

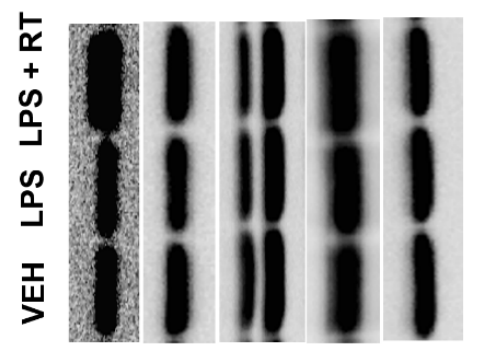

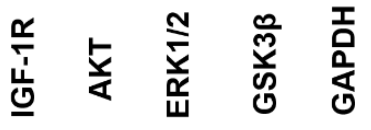
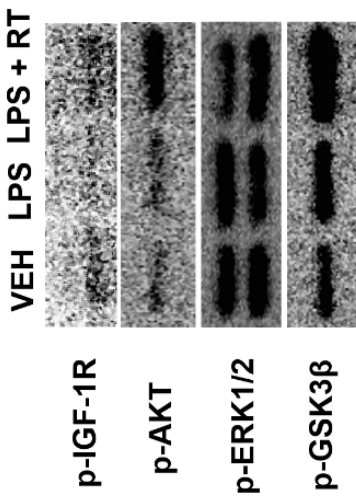

ш
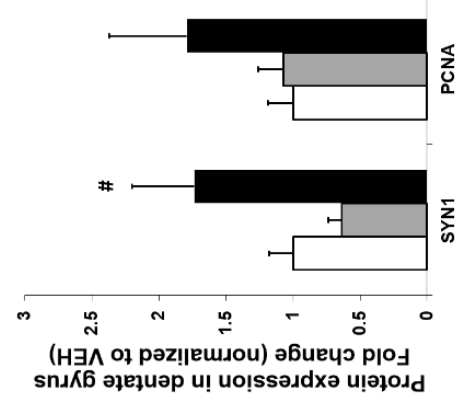

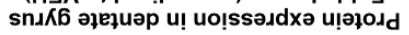


Figure 2.6. Effects of 6-weeks of LPS, with or without RT, on dentate gyrus molecular signaling. A) phosphorylation of IGF-1 signaling proteins, B) IGF-1 signaling total proteins, C) ratio of phosphorylation/total IGF-1 signaling proteins, and D) total protein markers of neuroplasticity and proliferation. E) Representative Western blots for proteins shown in panels A, B, and D. Protein expression was normalized to VEH controls. ${ }^{*}=p \leq 0.05$ significantly different from VEH group. \# = $p \leq 0.05$ significantly different from LPS group. One-way ANOVA, Holm-Sidak post-hoc test, $\mathrm{n}=$ seven per group. 


\section{Experiment two}

To characterize an early effect of RT by ladder climbing in the absence of LPS, Experiment two was performed with rats that underwent either one workout or three workouts (which were performed every other day during a single week). The general hypothesis underlying the short-term RT studies were that increases in IGF-1 signaling pathway would occur after only a single RT workout. Rats that underwent one workout showed no changes in phosphorylation of IGF-1R, AKT, ERK, or GSK3 $\beta$ 24-hrs post exercise compared to the SED group (Fig. 2.7A). Additionally, one workout did not result in any significant changes on PCNA or SYN1 protein concentrations compared with their sedentary counterparts (Fig. 2.7B). Conversely, three workouts (within one week) showed significant increases in phosphorylation of ERK1/2, SED vs RT was $1.00 \pm 0.06$ vs $1.34 \pm 0.14$ fold increase (Fig. $2.8 \mathrm{~A}$ ) and GSK3 $\beta$, SED vs RT was $1.00 \pm 0.16$ vs $2.09 \pm 0.38$ fold increase, respectively with $p \leq 0.05$ (Fig. 2.8A). There was also a significant increase in PCNA protein, SED vs RT was $1.00 \pm 0.15$ vs $1.72 \pm 0.27$ fold increase, respectively with $p \leq 0.05$ ) (Fig. 2.8B) after three RT workouts. Additionally, compared to SED group, the ratio between $\mathrm{p}$ GSK3 $\beta /$ GSK3 $\beta$ was significantly higher the RT group, SED vs RT was $1.00 \pm 0.17$ vs 3.21 \pm 0.71 fold increase. No changes in total proteins, phosphorylation of IGF-1R or AKT (Fig. 2.8A), nor changes in SYN1 protein (Fig. 2.8B) were observed. 


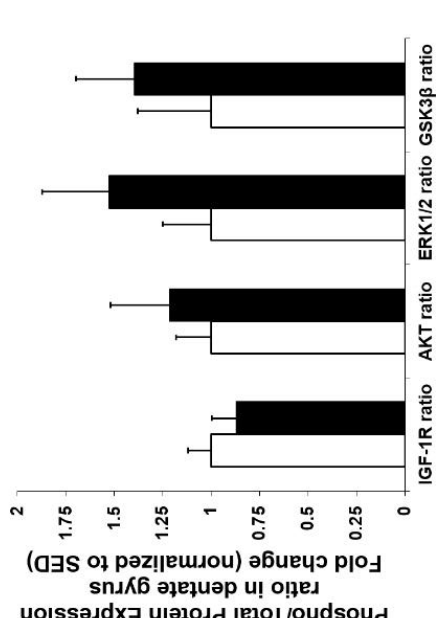

$\stackrel{\leftarrow}{\mathscr{\alpha}}$
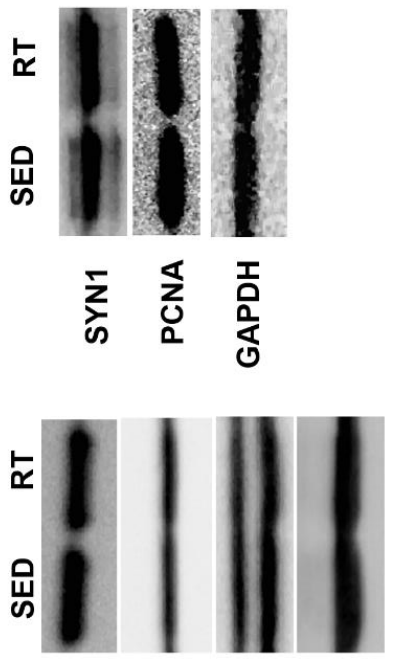

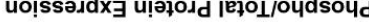
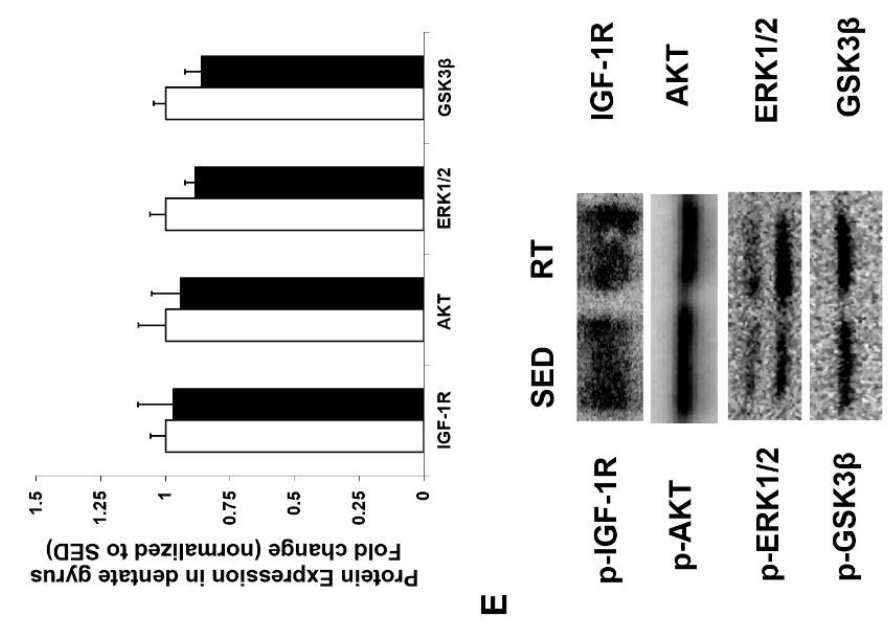

$m$

ш
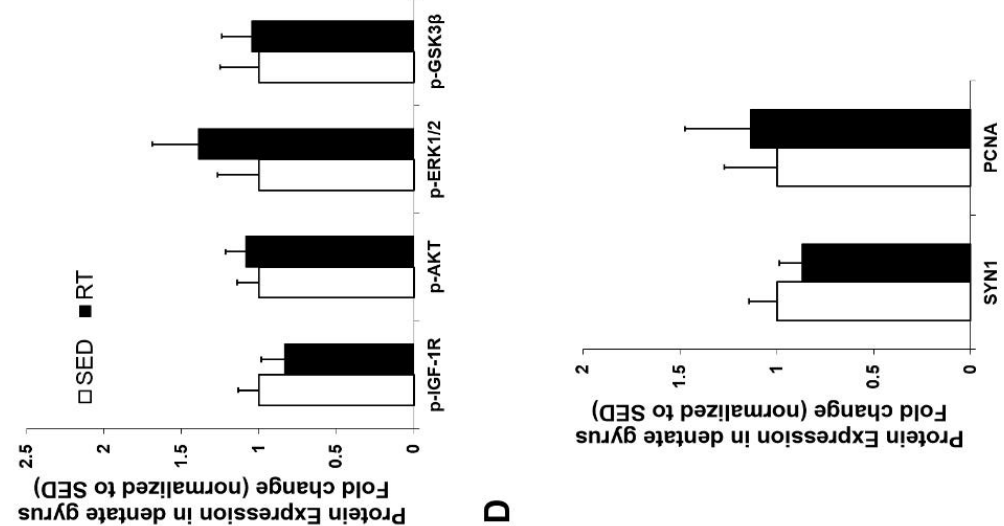

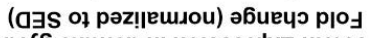

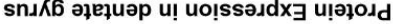

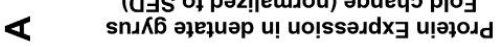


Figure 2.7. Effects of a single RT workout on dentate gyrus molecular signaling. A) phosphorylation of IGF-1 signaling proteins, B) IGF-1 signaling total proteins, C) ratio of phosphorylation/total IGF-1 signaling proteins, and D) total protein markers of neuroplasticity and proliferation. E) Representative Western blots for proteins shown in panels A, B, and D. Protein expression was normalized to values for sedentary (SED) control group. ${ }^{*} p \leq 0.05$ is significantly different from SED group. T-test, $n=$ seven per group. 


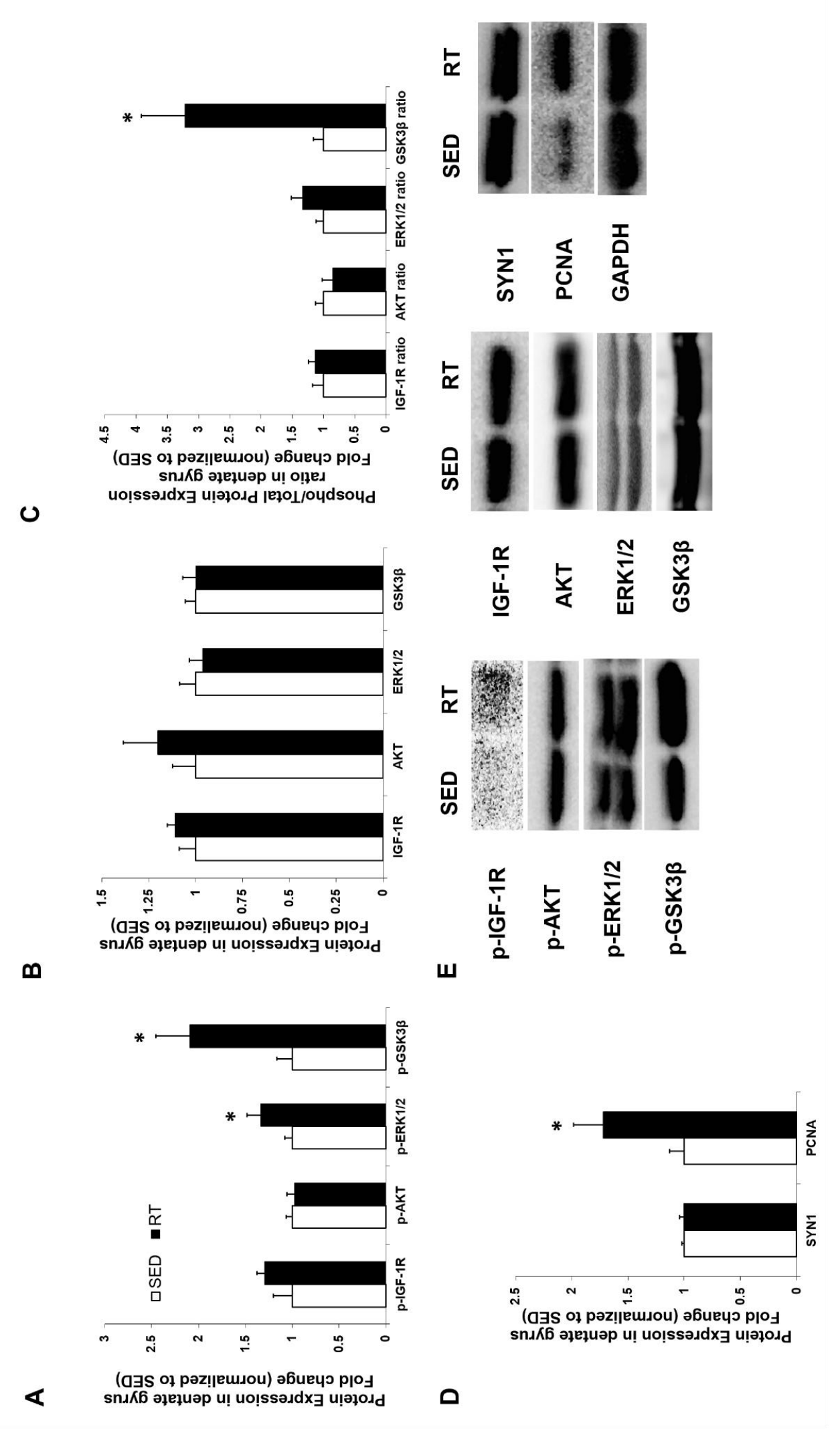


Figure 2.8. Effects of one week (3 RT workouts) of RT on dentate gyrus molecular signaling. A) phosphorylation of IGF-1 signaling proteins, B) IGF-1 signaling total proteins, C) ratio of phosphorylation/total IGF-1 signaling proteins, and D) total protein markers of neuroplasticity and proliferation. E) Representative Western blots for proteins shown in panels A, B, and D. Protein expression was normalized to values for sedentary (SED) control group. ${ }^{*} p \leq 0.05$ is significantly different from SED group. T-test, $n=$ seven per group. 


\section{Discussion}

The purpose of the current study was to investigate the effects of RT in animals subjected to LPS-induced MCI. We found that 1) RT ameliorated cognitive deficits induced by LPS injections in female rats; 2) 6-weeks of RT induced increases in strength and muscle mass, as well as specific IGF-I signaling molecules within the dentate gyrus in rats with $\mathrm{MCl}$; and 3) selected downstream molecular targets of IGF-1 signaling are increased within one week of RT (3 workouts).

Experiment one demonstrated efficacy of the RT protocol. We noted increases in: 1) lean mass percentage, 2) individual muscle wet weights, and 3) maximal strength, similar to effects noted in both animal (143) and human RT studies (95, 145, 146), suggesting verification of the ladder climbing protocol in the experimental design herein, as a suitable RT model for rats. To verify that LPS-induced neuroinflammation, dentate gyrus tissue proinflammatory transcripts were quantified through qRT-PCR. Similar to other reports (142), LPS-induced significant increases in TNF $\alpha$ and IL-1 $\beta$ compared to VEH (Fig. 2.2). Remarkably, improved cognition was associated with unaltered indices of LPS-induced, high inflammation, suggesting RT-induced benefits on neurocognitive function occur through alternative mechanisms.

Six-weeks of RT ameliorated cognitive deficits in spatial learning induced by LPS. Using the Barnes maze test, the LPS + RT group had a significantly shorter latency to locate the escape hole compared to the LPS group. In addition, error number decreased over a 4day period of learning in the LPS + RT group, providing evidence that decreased latencies in this group was in part related to improved memory performance. Human studies support this observation, showing that RT improves cognition in patients with $\mathrm{MCl}(95,101)$. Concurrently, animal studies examining the effects of RT show cognitive benefits (139) $(104,109)$. As others have stated $(109,135)$, the benefits in cognition and memory 
performance (a hippocampal-dominant process) might be due to RT-induced changes in IGF-1 signaling .

Other unique findings of this study are the molecular changes induced by RT for IGF-1 signaling proteins. Phosphorylation of IGF-1R was significantly increased in the LPS + RT group compared to LPS alone in the dentate gyrus, while phosphorylation of IGF-1R downstream proteins AKT and GSK3 $\beta(147,148)$ were significantly increased compared to both LPS or VEH in the dentate gyrus (Fig. 2.6A), suggesting AKT signaling is increased by RT. Interestingly, total IGF-1R was also significantly higher in the LPS + RT group compared to LPS, suggesting the increased IGF-1R signaling could likely be due to RTinduced increase in the IGF-1R. Since AKT signaling is involved in neuronal proliferation, survival, and plasticity $(136,137,149)$, we measured SYN1 and PCNA proteins in the dentate gyrus. SYN1 is a prevalent phosphoprotein involved in synaptic vessel trafficking and short-term synaptic plasticity within the central nervous system (150, 151), while PCNA is required for DNA replication (152) making it a useful marker of proliferation (153, 154) as its expression increases during the $S$ phase of the cell cycle in preparation of cell division (155). We hypothesized that SYN1 and PCNA would be increased in the dentate gyrus in response to RT, and found SYN1 to be significantly increased by RT, suggesting enhanced synaptic plasticity. However, no change in PCNA was observed. Previously, Cassihas et al. (109) showed increases in AKT signaling were coupled with increases in SYN1 protein in the whole hippocampus after eight weeks of RT in rats. Taken together, RT-induced IGF-1 activation of AKT and increased SYN1 could be a part of a primary mechanism by which RT contributes to improved cognition in $\mathrm{MCl}$.

Experiment two was designed to explore the effects of short-term RT (in the absence of LPS) at the molecular level by examining the dentate gyrus of two separate cohorts after one, or three, RT workouts. Although no changes in any of the determined proteins were induced by one RT workout. Importantly, three RT workouts (performed every other day) 
resulted in increased phosphorylation of downstream IGF-1 protein signaling (ERK1/2, and GSK3 $\beta$ ) compared to SED in the dentate gyrus, as well as increased proliferation marker, PCNA. However, no differences in SYN1 or IGF-1R phosphorylation were noted. The lack of change from either SYN1 or IGF-1R phosphorylation could be due, at least in part, to a greater duration training stimulus being required. These data suggest an augmentation of IGF-1 signaling and proliferation begins within one week of RT. Fernandes et al. (135) examined a single RT workout and found this was sufficient to induce changes in SYN1 in whole hippocampus, which conflicts with our one workout data on SYN1 in the dentate gyrus portion of the hippocampus. The different results could be explained, at least in part, by the fear conditioning (conditioning that utilizes electric shock for aversive learning, distinct from Barnes maze spatial learning) that rats experienced in Fernandes et al. study (135).

\section{Considerations}

When evaluating the divergent molecular responses to one, three and $18 \mathrm{RT}$ workouts, with the latter being in rats injected with LPS, there are a number of considerations. For example, PCNA was increased following three RT workouts within a single week in control rats, but not 18 RT workouts in rats injected with LPS. Reports show that LPS can lead to a reduction in proliferation within the dentate gyrus (134), which might attenuate RTinduced proliferation, and possibly explain why no difference in proliferation was observed after 6-weeks of LPS + RT, while PCNA did increase after one week of RT in absence of LPS. We speculate that not observing changes in PCNA could be due to the LPS-induced $\mathrm{MCl}$. In addition, as the RT loading was modified weekly (on an individualized basis) to adjust for increases in strength that occurred during the training program, it is also possible that RT-induced molecular adaptations and regulation of IGF-1 signaling were affected differently by one, three and $18 \mathrm{RT}$ workouts. This highlights the potential importance of 
performing regular RT and employing an optimal progressive RT protocol in order to maximize the full neurocognitive benefits of this training modality. Furthermore, the ladderclimbing RT model was completed in a voluntary, progressive manner, analogous to human RT, with step-wise increases in loading occurring at the beginning of each training week. These load increases were individually tailored to each rat's individual adaptability to the training programs, similar to the heterogeneity in response noted in human RT studies (156). Another important advantage of this ladder-climbing model is the voluntary nature, without the need for electrical shock and/or forced exercise, which could evoke significant stress and inflammatory responses in the dentate gyrus (157-160), as stress and inflammation are contraindicated to the prevention of $\mathrm{MCl}$.

Others have reported TNF- $\alpha$ and IL-1 $\beta$ are induced by LPS, which can lead to $\mathrm{MCI}$ (78, 161, 162). However, we noted that RT did not alter elevated inflammatory cytokine levels, suggesting that RT-induced improvements in cognition occurred independent of changes in expression of these inflammatory markers. Additionally, although cognitive deficits were not associated with significantly impaired expression of plasticity after the 6-week intervention period, it is possible LPS may have decreased synaptic plasticity markers such as SYN-1 immediately after injection; however, compensation may have occurred with time, leading to the lack of reduced SYN-1 after six weeks. Nevertheless, the improved cognition was associated with increased synaptic plasticity as well as increased IGF-1/AKT signaling.

In addition, there are limitations to consider. First, we used female rats in the current study, as females represent the $62 \%$ of U.S. AD patients (8). However, it is unclear whether males would respond similarly to this RT program. We did not perform DEXA scanning prior to the intervention, as this would require the rats being exposed to isoflurane prior to cognitive testing, which has been shown to induce cognitive dysfunction (163). An additional limitation was that the control group did not perform a full training 
session in the absence of a training load, and instead performed one set, climbing $27 \mathrm{~cm}$. Thus, one might interpret our RT model as exhibiting an aerobic component. However, since the RT group only climbed 5-10 seconds per set, totaling approximately 40-80 seconds per workout, we speculate that any aerobic adaptation probably did not contribute to the effects noted.

In conclusion, we have demonstrated that progressively-weighted ladder climbing as a rodent model of RT ameliorates LPS-induced cognitive deficits, and these improvements in cognitive function occurred in concert with RT-induced IGF-1 signaling. This model offers a potential therapy, which may prevent or delay the onset of $\mathrm{MCl}$ in neurodegenerative diseases that warrants further investigation. 


\section{Chapter Three: Resistance-exercise training ameliorates LPS-induced neuroinflammation and cognitive deficits by three weeks in Wistar rats}

Taylor J. Keltya, Xuansong Mao a, Nathan R. Kerr'a David T. Rouse ${ }^{\mathrm{a}}$, Thomas E. Childs ${ }^{\mathrm{a}}$, Gregory N. Ruegseggere and Frank W. Bootha, b, c, d

${ }^{a}$ Department of Biomedical Sciences

${ }^{\mathrm{b}}$ Department of Nutrition and Exercise Physiology

${ }^{c}$ Department of Medical Pharmacology and Physiology

${ }^{d}$ Dalton Cardiovascular Research Center

University of Missouri

Columbia, Missouri 65211, U.S.A.

e Department of Health and Human Performance

University of Wisconsin-River Falls

410 S 3rd St, River Falls, WI 54022, U.S.A. 


\section{Abstract}

Neuroinflammation is an early detectable marker of mild cognitive impairment, the transition state between normal cognition and dementia. Resistance-exercise training can attenuate the cognitive decline observed during mild cognitive impairment. However, the underlying mechanisms by which resistance-exercise training attenuates this cognitive decline is largely unknown. Here, we tested if three weeks of resistance-exercise training in Wistar rats could ameliorate lipopolysaccharide-induced neuroinflammation and cognitive impairment and if it was time dependent. To test this, 5-week old female rats received intracerebroventricular injections of lipopolysaccharides to induce neuroinflammation and mild cognitive impairment. Rats then underwent three weeks of progressive ladder climbing to mimic resistance-exercise training. Cognition and anxietylike behavior were assessed towards the end of the training period. Inflammation and IGF1 signaling markers were determined at one and 24-hours post resistance-exercise training. Resistance-exercise training restored lipopolysaccharide-induced deficits in novel object recognition. Additionally, resistance-exercise training improved cognitive flexibility, regardless of lipopolysaccharide injection. Lipopolysaccharides increased proinflammatory transcripts in the dentate gyrus that were attenuated by resistanceexercise training in a phasic time-dependent manner. Lipopolysaccharides increased IGF$1 \mathrm{R}$ signaling and resistance-exercise training regulated IGF-1 and IGF-1R in the dentate gyrus. Therefore, three weeks of resistance-exercise training alleviated lipopolysaccharide-induced neuroinflammation in a time dependent manner that associated with ameliorated cognitive impairment. This data indicate resistance-exercise training could be a powerful intervention to treat neuroinflammation that works in a time dependent manner. Additionally, we provide evidence that ladder-climbing model of resistance-exercise training in rats can improve multiple forms of cognition as early as three weeks. 


\section{Introduction}

Dementia is an age-related disease defined by the loss of cognition that impedes an individual's daily functioning capabilities (1). Although rising dementia prevalence seems to be slowing for reasons still not fully understood (164), the absolute dementia prevalence is still relatively high. The global prevalence of dementia in 2015 was estimated at 47 million cases which is predicted increase to 75 million by 2030 , making dementia a major concern for the future of global public health (165). Dementia can be divided into two categories the "reversible" dementias such as those of vascular or metabolic nature or “irreversible” such as Alzheimer's disease, Parkinson's disease, and amyotrophic lateral sclerosis. The irreversible dementias are also classified as neurodegenerative dementias due to the loss neurons (1). Despite advancement in technologies, there has been little pharmaceutical success to treat neurodegenerative dementia as of recent (166). However, a phase two trial of 257 patients with early symptomatic AD recently reported modestly lower cognitive decline and function decline compared to placebo after 18 months (167). Although trials like these are promising, at present, no cure for neurodegenerative dementia exist. Since reversing neurodegenerative dementia is challenging, prevention could provide an effective alternative to attenuate the predicted future increase in neurodegenerative dementia. The transition state between normal cognitive function and dementia, known as mild cognitive impairment (MCl) (168), could offer a potential therapeutic window to prevent neurodegenerative dementia.

$\mathrm{MCl}$ is a measurable symptomatic precursor of dementia (169). Positron emission tomography (PET) with inflammatory tracers have proposed neuroinflammation as one of the earliest detectable biomarkers for dementia progression, where severity of cognitive decline correlates with neuroinflammatory levels $(75,76)$. Intracerebroventricular injections of lipopolysaccharides (LPS), components of the gram-negative bacteria known to trigger an immune response, are widely used in animal studies to mimic 
neuroinflammation (78). Lateral ventricle injection of lipopolysaccharides elevates proinflammatory cytokines representing the early neuropathology observed in patients with $\mathrm{MCl}$ (75). Further support of LPS models for $\mathrm{MCl}$ comes from the endotoxin hypothesis which posits increased excretion of LPS from inflamed gut/gums facilitates the neurodegenerative process (88). Combined, these studies suggest LPS-induced neuroinflammation is a relevant model to study $\mathrm{MCl}$.

Clinical studies in patients with $\mathrm{MCl}$ have revealed resistance-exercise training (RT) as a potential intervention to ameliorate $\mathrm{MCl}$ (170). With that basis, the current study assessed if RT differentially effects types of cognitive function such as object recognition memory, spatial memory, and cognitive flexibility with and without LPS-induced neuroinflammation. To the best of our knowledge, only one other lab has examined the effect of RT on neuroinflammation (102). Therefore, to provide further insight into the effect of RT on neuroinflammation, the present study also tested if RT amelioration of neuroinflammation is an immediate or delayed effect (i.e. one or 24-hours). Additionally, insulin-like growth factor-1 (IGF-1) signaling was assessed as a potential mechanism due to the reported alterations of both RT and LPS on IGF-1, inflammation and cognition (139, 171).

\section{Materials and Methods}

Animal husbandry

The University of Missouri Institutional Animal Care and Use Committee (IACUC), which operates under the U.S. Guide for the Care and Use of Laboratory Animals, approved all procedures prior to the start of experiments. Female, 5-week-old, Wistar rats were obtained using breeder Wistar rats, approximately 65-days of age, obtained from Charles River Laboratories (Wilmington, MA). Pups were weaned at 21 days of age and were grouped housed until surgery. After surgery rats were single-housed for the remainder of the experiment. Rats were maintained on a 12-hr:12-hr light/dark cycle at $25^{\circ} \mathrm{C}$ and 
received food and water (Formulab Diet 5008; Purina, St. Louis, MO) ad libitum during the entire length of the study. Rats were divided into four groups: VEH + SHAM group (received vehicle injections and sham RT), LPS +SHAM group (received LPS injections and sham RT), VEH + RT (received vehicle injections and three weeks RT), and LPS + RT group (received LPS injections and three weeks RT). Rats were divided equally into groups based on average bodyweight at the start of the study $(n=12)$ per group). The timeline and groups are shown in Fig. 3.1a. 
A Groups:

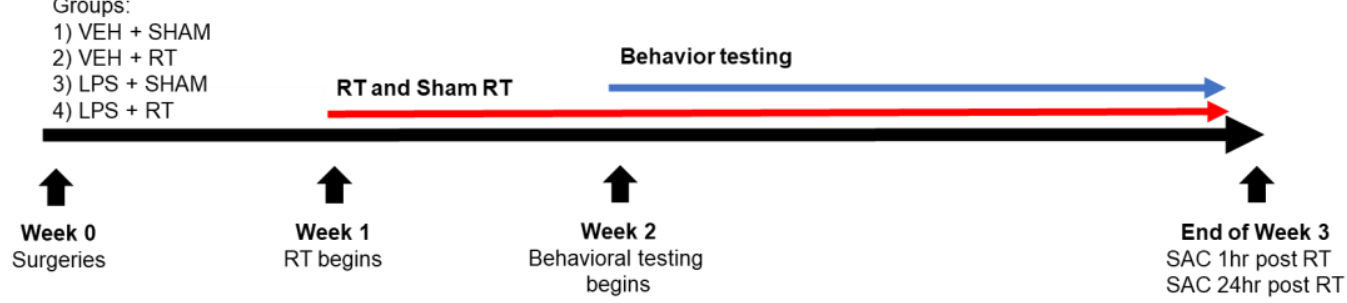

B

C
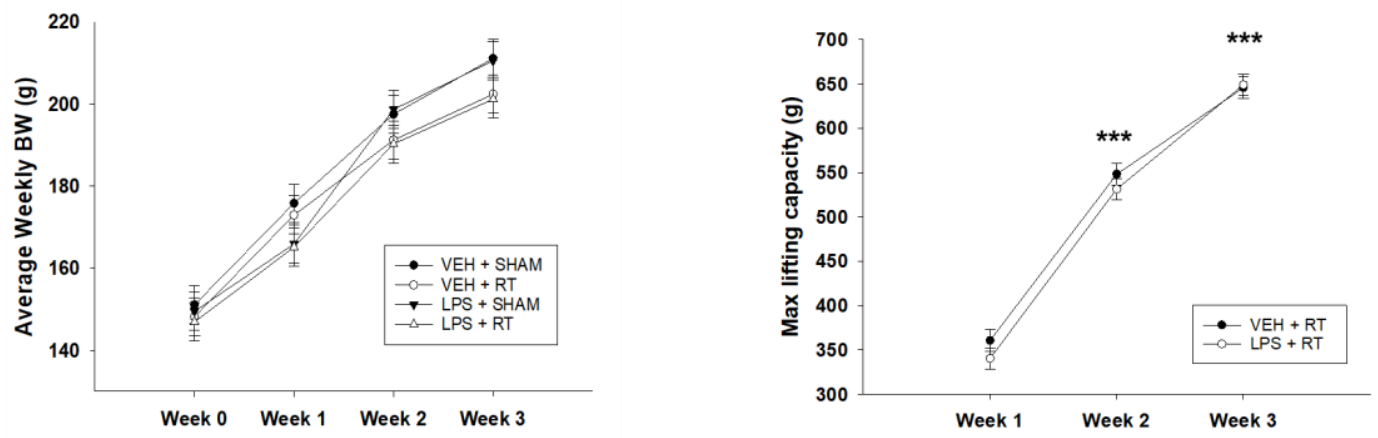

Figure 3.1 Timeline and changes of body weight (BW) and lifting capacity during the study.

A) Timeline for the study, where all four groups underwent surgeries, RT or SHAM training, and behavioral testing. B) Average weekly BW for each week of the study. C) Average maximum lifting capacity each week based on the amount of weight a rat could lift in a given week combined with that individual rat's BW. ${ }^{* * *}$ denotes a significant effect $(\mathrm{p} \leq$ .001). Two-way ANOVA was used for comparison between VEH + RT compared to LPS + RT ( $n=12)$ rats over the three weeks of RT. Holm Sidak was used post hoc for multiple comparison procedure. 


\section{Intracerebroventricular direct injections}

To induce brain inflammation, rats in experiment one received intracerebroventricular injections of LPS similar to previous experiments (172). All rats were anesthetized using $2-5 \%$ isoflurane, placed in a stereotactic frame (David Kopf Instruments, Tunjunga, CA), and prepped for surgery. Rats received banamine at a dosage of $1 \mathrm{ml} / \mathrm{kg}(2.5 \mathrm{mg} / \mathrm{ml})$ as an analgesic. Coordinates for bilateral injections were $0.8 \mathrm{~mm}$ posterior to bregma, $1.5 \mathrm{~mm}$ lateral to the midline suture (on either side), and $3.6 \mathrm{~mm}$ below the skull surface. Using a 25-ul Hamilton syringe (Hamilton Co., Reno, NV), $0.9 \%$ saline solution or LPS (Escherichia coli O55:B5, Sigma, St. Louis, MO) bilateral injections of 2ul of LPS solution (5ug/ul) per side at a rate of $1 \mathrm{ul} / \mathrm{min}$ followed by a 3 -minute waiting period for solution to be absorbed in the lateral ventricles. Throughout the surgery, all rats breathing and toepinch reflex were monitored to assure proper anesthesia. Rats were monitored over the five days of recovery before RT began.

\section{Resistance-exercise training protocol}

As previously published (172), rats started at the bottom of the ladder then would freely, without electric shock, climb 100-cm (at an $80^{\circ}$ incline) into a housing chamber where the rat would rest for one minute between each climb. During RT, rats' would have weights in a holding apparatus attached to the base of the tail with athletic tape. The RT group of rats trained three days a week for three weeks. The first day of each week of training was used to determine the maximum lifting capacity of each rat. Day one consisted of a warmup climb with no weight, followed by two lifts of $75 \%$ of previous max, followed by an approximate $10 \%$ increase in weight per climb until the rat was unable to complete a climb to the top of the ladder (considered failure). Note: the first climbing session considered the rats bodyweight as the maximum lifting capacity. The following two days of ladder climbing each week were based on the maximum lifting capacity to create a progressive and 
individualized workout plan for each rat. The following days of that week included a warmup climb followed by eight subsequent climbs at $50,50,75,75$, and $90,100,100,100 \%$ of the rat's maximum lifting capacity. Rats received 1-2 days of recovery between workouts to simulate human conditions (173). Rats that did not receive RT (SHAM groups) instead received a sham-RT where rats climbed $27-\mathrm{cm}$ up the ladder using a sham start platform without weights attached to the tail.

\section{Elevated and maze testing}

The elevated plus maze is a common method in rodents for measuring anxiogenic behavior, which is based on a rodent's aversion to open spaces (174). The elevated plus maze is a plus shaped platform consisting of four arms. Two of the arms on the platform have walls were considered "closed" arms and two arms do not have walls and were considered "open" arms. Each arm of the maze is 43-cm long $\times 14-\mathrm{cm}$ wide, closed platforms have $22-\mathrm{cm}$ tall walls on all three sides. The entire apparatus is $63-\mathrm{cm}$ off the ground. All animals were brought into testing room a minimum of 20 minutes before experiment started to acclimate them to the room. At the beginning of testing, thermometer, lux, and decibel meter were used to monitor temperature, light, and sound, respectively. Each session began by gently placing the rat at the junction of the open and closed arms. The rat was allowed to freely explore the maze for five minutes while time spent either the closed arm or open arm was recorded. To reduce odors $70 \%$ ethanol was used to clean the apparatus between each rat. Non-biased AnyMaze behavior tracking software was used to record all data (Stoelting Co., Wood Dale, IL). The ratio of open or closed arm times to total time spent expressed as a percent was analyzed to provide a measurement of anxiety-like behavior.

\section{Barnes maze testing}


The Barnes maze test is a cognitive assessment to measure spatial learning/memory in rodents based on the rodents' aversion to an open and well-lit area (175). A rotating circular gray Barnes maze platform (122-cm in diameter) with 20 holes (each being 10-cm in diameter) was used to test spatial learning/memory behavior. Beneath one of the 20 holes was a dark goal box (30-cm in length X $15-\mathrm{cm}$ wide $X 13-\mathrm{cm}$ high) for the rat to climb into and escape from the aversive open and well-lit platform. The $76-\mathrm{cm}$ high circular platform allowed each rat to be assigned a different target hole to escape into, which helped eliminate odor cues when testing, but also controlled for any biases from visual cues by keeping the same location of the target hole for each rat. Temperature, light and noise were also controlled for during testing. Testing occurred during the rats' light cycle for five consecutive days (two trials per day). Before the first trial rats are placed in the goal box for two minutes to help associate the goal box as a safe environment. During each trial, the rat was placed in the center of the maze under a start box for 30 seconds then was given five minutes allowed to explore the maze freely. If in this time the rat was unable to locate and climb into the goal box, the rat was gently guided into the goal box. The rat was allowed 30 seconds to stay in the goal box to associate it as a safe place. Next, the rat was taken out of the goal box and was placed back into its home cage and the Barnes maze platform was cleaned with $70 \%$ ethanol to help eliminate odor cues. The time taken to find and step into the whole (latency to goal box) and number of errors (nose pokes into the incorrect hole) was recorded. During reversal testing, the hole was placed in a new location $180^{\circ}$ from the original location. The rat underwent reversal testing following the last day of Barnes maze testing. The reversal testing lasted three consecutive days (2 trials per day); following a similar protocol to Barnes maze testing. Design and methods were adapted from previous experiments (172). Non-biased AnyMaze tracking software (Stoelting, Wood Dale, IL) was used to record Barnes maze data. 


\section{Novelty object recognition testing}

The novelty-object recognition test is a behavioral test used to measure memory in rodents. During the test, rats are placed in a box $(60-\mathrm{cm}$ in length $X 60-\mathrm{cm}$ width $X 46-\mathrm{cm}$ height) and allowed to explore similar and novel objects. The test is based on the natural proclivity of a rodent to explore novel objects (176). Testing consisted of three phases where rats undergo habituation, familiarization, and novelty object recognition testing. During habituation, rats were given five minutes to explore the box freely to acclimate to the new environment. Familiarization takes place 24-hours after habituation where rats are given up to 10-minutes to explore the box with two identical objects sitting 5 -cm from the wall on each side. Novel object recognition testing is performed one hour later where one of the similar objects is replaced by a novel object and given up to ten minutes to explore. The experiment was stopped after the rat had explored the objects for 20 seconds or when the 10-minute time period was over (i.e., the maximum session time). The time spent exploring each object is recorded and analyzed (time spend exploring novel object/ time spent exploring both objects in total) to create an object preference. Temperature, light, and sound was monitored during testing and the novelty-object recognition box was cleaned with $70 \%$ ethanol between sessions to help eliminate odor cues. These methods were modified from Leger et al.(176).

\section{Tissue Collection}

Tissue collection took place at 1-hour and 24-hours after the last RT or sham workout. Rats were euthanized via carbon dioxide asphyxiation, followed by extraction of the brain. Dentate gyrus punches that were $2 \mathrm{~mm}$ thick and $3-\mathrm{mm}$ in diameter were taken from coronal brain slices sliced using a brain matrix (Braintree Scientific, Braintree, MA) 
identified using a rat brain atlas (177). Dentate gyrus punches were flash frozen with liquid nitrogen and stored at $-80^{\circ} \mathrm{C}$ until processing.

RNA Isolation, cDNA Synthesis, and qRT-PCR

1-hour or 24-hours after the last RT workout, rats were euthanized, and the dentate gyrus was removed as described above. Dentate gyrus RNA isolation, cDNA synthesis, and qRT-PCR were performed as described previously (172). In short, dentate gyrus punches were place in TRIzol (Invitrogen, Carlsbad, CA) and homogenized three times for 1-minute at $25-\mathrm{Hz}$ using RNase-free, stainless steel beads and a Tissuelyser (Qiagen, Germantown, MD). Samples were cooled in ice for 1-minute after each homogenization to prevent RNA degradation. Using TRIzol protocol, RNA was extracted and quantified using a Nanodrop 1000 (Thermo Scientific, Waltham, MA). Following quantification, cDNA synthesis was completed by treating RNA with DNase using DNase I (Thermo Scientific) and reverse transcribed using a High Capacity cDNA Reverse Transcription Kit (Applied Biosystems, Carlsbad, CA). Gene-specific primers were constructed using Primer3 (Table 3.1) and used for qRT-PCR. cDNA from each sample was assayed in duplicate for target genes in Table 3.1 using iTaq Universal SYBR green Supermix (Bio-Rad Laboratories, Hercules, CA) and RNA expression values were normalized to $18 \mathrm{~S}$ and quantified by the $2^{\Delta \Delta} \mathrm{Ct}$ Method. To avoid statistical analysis on logarithmically transformed data, changes in the $\Delta \mathrm{CT}$ was used for statistical analysis. Graphs are expressed as fold change (normalized to VEH). 


\begin{tabular}{|llll|}
\hline Gene & Forward $\left(\mathbf{5}^{\prime} \mathbf{- 3} \mathbf{\prime}\right)$ & Reverse $\left.\mathbf{( 5}^{\prime} \mathbf{- 3} \mathbf{\prime}\right)$ & Accession number \\
\hline 18S & GCTCGCTCCTCTCCTACTTG & GATAAATGCACGCGTTCCCC & NR_046237.2 \\
\hline TNF- $\alpha$ & AACACACGAGACGCTGAAGT & TCCAGTGAGTTCCGAAAGCC & NM_012675.3 \\
\hline IL-1 $\beta$ & TGACTTCACCATGGAACCCG & GACCTGACTTGGCAGAGGAC & NM_031512.2 \\
\hline IL-6 & CAGTTGCCTTCTTGGGACTGA & GAAGTCTCCTCTCCGGACTTG & NM_012589.2 \\
\hline GFAP & TTGACCTGCGACCTTGAGTC & GAGTGCCTCCTCCTGGTAACTCG & NM_017009.2 \\
\hline IGF-1 & CGTCTAGGCCATTAGCCCTG & AGGGGCGTTAAAACTTGGGT & NM_178866.4 \\
\hline IGF-1R & CTGGGTGTAGTATCCCAAGGC & GGACTAGATTATTCTCCACCTCTGG & NM_052807.2 \\
\hline
\end{tabular}

Table 3.1. Primer sequences used for gene expression analysis by qRT-PCR. 


\section{Immunoblotting}

Methods for immunoblotting were similar to previous studies(172). Briefly, dentate gyrus rich plugs underwent protein isolation where protein concentration was obtained using the BCA assay (Pierce Biotechnology, Rockford, IL). Fifteen micrograms of protein were loaded for electrophoresis and transferred to nitrocellulose membrane. Immunoblots were incubated with Ponceau S (Sigma, St. Louis, MO) to verify equal loading in each lane. After blocking with 5\% milk and washing, blots were bathed in primary antibody overnight at $4^{\circ} \mathrm{C}$. All primary antibodies were purchased from Cell Signaling (Danvers, MA), which included IGF-1 receptor beta (IGF-1Rß) (1:2000), Phospho-p44/42 mitogen-activated protein kinase (Erk1/2) (Thr202/Tyr204) (pERK) (1:2000), p44/42 MAPK (Erk1/2) (ERK) (1:2000), protein kinase B (AKT) (1:2000), phospho-AKT (pAKT) (Ser473)(1:1000), glycogen synthase kinase $3 \beta(G S K-3 \beta)$ (1:2000), phospho-GSK (pGSK) (Ser9) (1:1000), Glial fibrillary acidic protein (GFAP) (1:2000), Glyceraldehyde 3-phosphate dehydrogenase (GAPDH) (1:50,000). Phospho-IGF-1Rß (pIGF-1R) (y1135/1136) (1:500) antibody was applied for three days at $4^{\circ} \mathrm{C}$ before applying secondary antibody. Horseradish peroxidase-conjugated secondary (1:1000) (Cell Signaling, Danvers, MA) was applied for one hour at room temperature the following day. Horseradish peroxidaseconjugated substrate (Sigma, St. Louis, MO) was applied for five minutes before exposure. A Kodak 4000R Imager with Molecular Imagery Software (Kodak Molecular Imaging Systems, New Haven, CT) was used to determine band densitometry. Molecular weight was estimated using Precision Plus Protein Standards (Bio-rad Laboratories, Hercules, $\mathrm{CA})$.

\section{Statistical Analysis}

All analytical procedures were performed using SigmaPlot 14.0 (Systat Software, San Jose, CA). One-way ANOVA was used for comparison of trials during Barnes maze and 
reversal testing. Two-way ANOVA was used for all other data analyses. Trial one of Barnes maze and reversal testing were removed from analysis as trial one does not represent learning/memory measurements. Holm-Sidak test was applied post hoc for all pairwise multiple comparison procedures. A $p \leq .05$ was used as the significance level. All values are expressed as means and standard errors.

\section{Results}

Confirmation of $R T$ regiment

To further verify RT, a two-way ANOVA was used to analyze maximum lifting capacity during the three weeks of RT. Analysis of maximum lifting capacity revealed a main effect of weeks, $F(2,66)=311.808, p \leq .001$. Post hoc analysis revealed increased lifting capacities for each week. Week two $(M=540.033, S E M=8.512)$ was significantly higher than week one $(M=350.604, S E M=8.512), t=15.735, p \leq .001$; and week three $(M=$ $647.483, \mathrm{SEM}=8.512)$ was significantly higher than week two $(M=540.033$, SEM $=$ 8.512), $t=8.926, p \leq .001$ (Fig. 3.1c).

LPS and RT differentially effects novel object recognition memory, spatial memory, and cognitive flexibility

To assess novel object recognition memory, a two-way ANOVA was used to examine the effect of LPS and RT. Analysis of novel object preference revealed a significant interaction, $\mathrm{F}(1,43)=8.767, \mathrm{p}=.005$. Post hoc analysis revealed LPS + SHAM had a significantly decreased preference to the novel object $(M=0.523$, SEM $=0.0279)$ compared to $\mathrm{VEH}+\mathrm{SHAM}(\mathrm{M}=0.685, \mathrm{SEM}=0.0279), \mathrm{t}=4.105, \mathrm{p} \leq .001$ (Fig. 3.2b) Post hoc analysis also revealed LPS + SHAM had a significantly decreased preference to the novel object $(M=0.523, S E M=0.0279)$ compared to $L P S+R T(M=0.623, S E M=$ 
0.0291 ), $\mathrm{t}=2.470, \mathrm{p}=.018$ (Fig. 3.2b). These data indicate that three weeks RT ameliorated object recognition deficits induced by LPS.

To verify Barnes maze and reversal testing, a one-way ANOVA was used to examine the effect of trial for latency and number of errors. Analysis revealed a main effect of trial for latency $(F(9,469)=20.411, p \leq .001)$ and errors $(F(9,470)=11.733, p \leq .001)$ made during Barnes maze testing. Post hoc analysis revealed trial ten time $(M=13.104$, SEM $=1.738)$ and errors $(\mathrm{M}=1.677, \mathrm{SEM}=0.28)$ were significantly lower than trial one time $(M=92.688, S E M=13.101 ; t=9.140, p \leq 0.001)$ and errors $(M=6.229, S E M=0.807 ; t=$ $6.675, p \leq 0.001)$ (Fig. 3.2c \& d). Analysis revealed a main effect of trial for latency $(F(5$, $281)=10.182, p \leq .001)$ and errors $(F(5,281)=8.229, p \leq .001)$ made during reversal testing. Post hoc analysis revealed trial six time $(M=9.375, \mathrm{SEM}=1.369)$ and errors ( $\mathrm{M}$ $=1.021, \mathrm{SEM}=0.222)$ were significantly lower than trial one time $(\mathrm{M}=32.875, \mathrm{SEM}=$ $4.251 ; t=6.307, p \leq 0.001)$ and errors $(M=4.583, S E M=0.638 ; t=5.847, p \leq .001)($ Fig. $3.2 g \& h)$.

To assess spatial learning and memory, a two-way ANOVA was used to examine the effect of LPS and RT during Barnes maze testing. Analysis of errors revealed a main effect of LPS, $F(1,428)=4.340, p=.038$. Post hoc analysis revealed LPS led to a significant increase number of errors $(M=2.847, S E M=0.217)$ compared to VEH $(M=2.208$, SEM $=0.217), t=2.083, p=.038$ (Fig. 3.2f). These data indicate LPS induced a spatial learning and memory deficit.

To assess cognitive flexibility, a two-way ANOVA was used to examine the effect of LPS and $R T$, during reversal testing. Analysis of errors revealed a main effect of $R T, F(1,235)$ $=9.778, p=.002$. Post hoc analysis revealed three weeks of RT significantly decreased the number of errors made $(M=1.292$, SEM $=0.236)$ compared to SHAM $(M=2.333$, $\mathrm{SEM}=0.235), t=3.127, p=.002$ (Fig. 3.2j). These data indicate three weeks $R T$ improved cognitive flexibility. 
Considering the relationship between anxiety and cognition (178), we use elevated plus maze to determine if there were any changes in anxiety that may influence our cognitive behavioral tests. We found no change in anxiety-like behavior from RT or LPS, suggesting anxiety did not account for the changes we observed in cognition. 
A

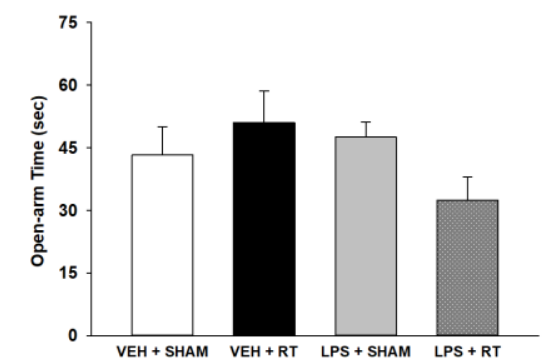

C

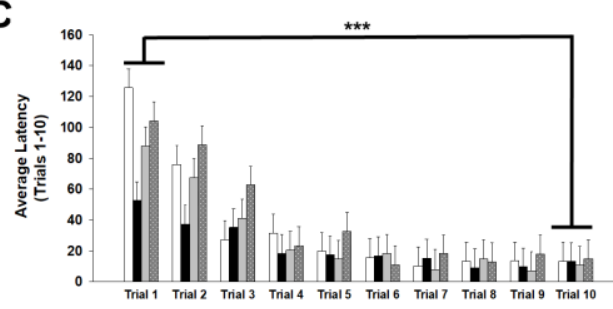

E

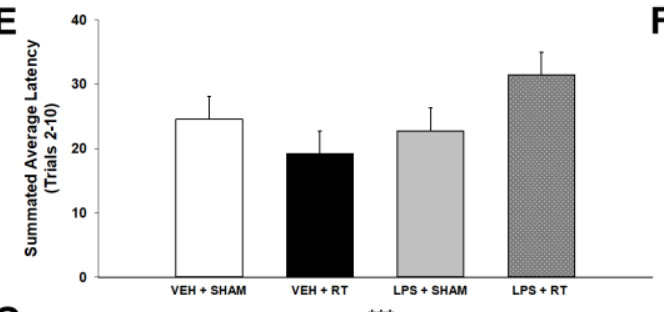

G
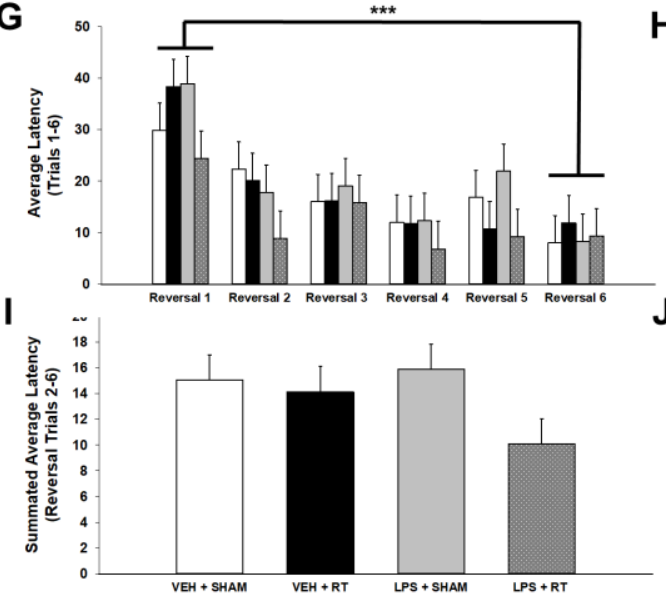

B

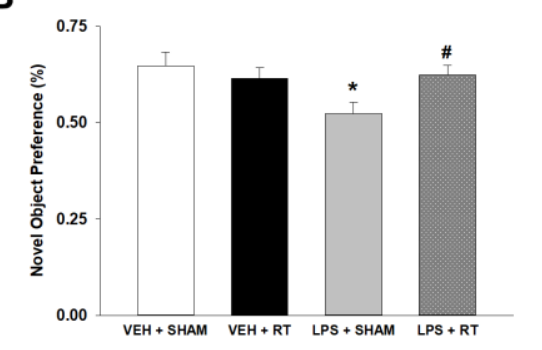

D
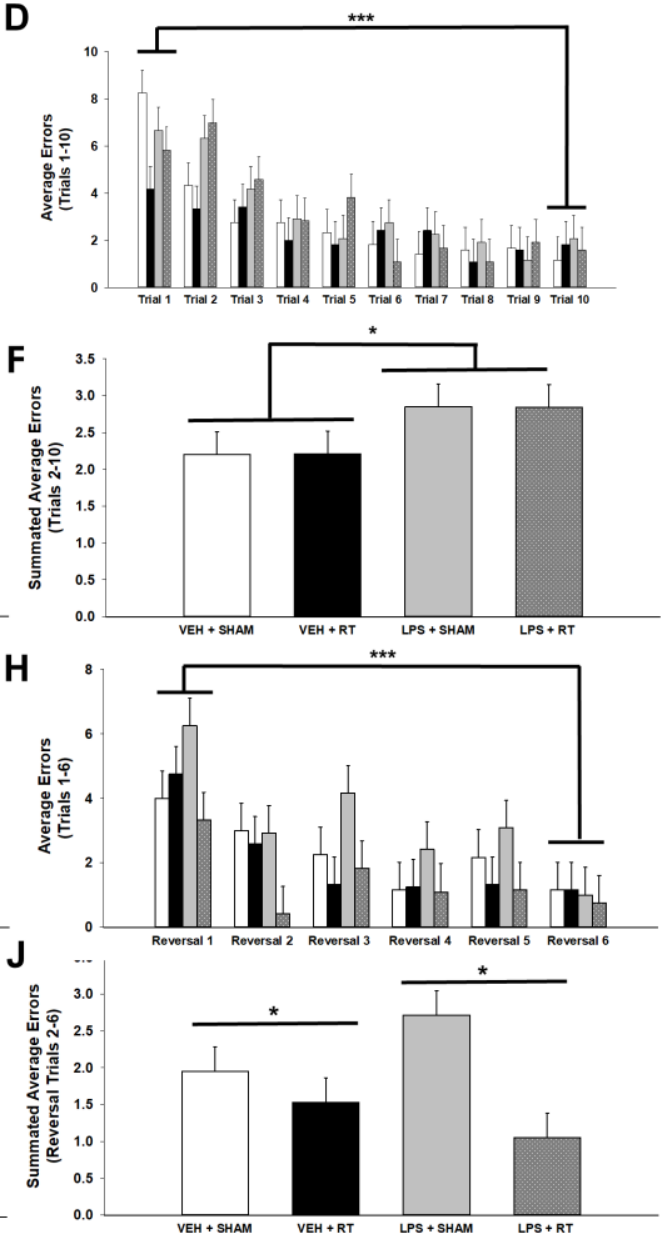

Figure 3.2 Behavioral testing revealed LPS impaired cognition while RT was able to improve cognition. A) Time spent in open arm of elevated plus maze. B) Preference of object during novel object recognition. C) Average latency time (seconds) to find goal box during five days of Barnes maze testing (two trials/day). D) Average errors made before finding goal box during five days of Barnes maze testing (two trials/day). E) Summated 
average latency (excluding Trial 1) during Barnes maze testing. F) Summated average errors (excluding Trial 1) during Barnes maze testing. G) Average latency time (seconds) to find goal box during three days of reversal testing (two trials/day). H) Average errors made before finding goal box during three days of reversal testing (two trials/day). I) summated average latency (excluding Trial 1) during reversal testing. J) Summated average errors (excluding Trial 1 ) during reversal testing. * denotes a significant effect ( $p$ $\leq .05)$.\# denotes the reversal of a significant effect $(p \leq .05)$. ${ }^{* *}$ denotes a significant $(p \leq$ .001). For differences between trials, a one-way ANOVA was used for comparison between groups. For all other analyses, a two-way ANOVA was used for comparison between groups $(n=11-12)$. Holm Sidak test was used post hoc for multiple comparison procedure. 
$R T$ attenuates LPS-induced neuroinflammation in the dentate gyrus

To assess the effect of RT on neuroinflammation, a two-way ANOVA was used to examine the effect of LPS and RT on inflammatory transcripts in the dentate gyrus at one and 24hours post RT.

Analysis of inflammatory markers at 1 -hour post RT revealed a main effect for in inflammatory transcripts TNF- $\alpha$ (Tumor necrosis factor-alpha) $(F(1,19)=13.783, p \leq$ $.001)$, IL-1 $\beta$ (interleukin-1- beta) $(F(1,19)=17.696, p \leq .001)$, and IL-6 $(F(1,19)=27.198$, $\mathrm{p} \leq$. 001). Post hoc analysis revealed LPS significantly increased TNF- $\alpha$ transcript $(\mathrm{M}=$ $0.591, \mathrm{SEM}=0.164)$ compared to $\mathrm{VEH}(\mathrm{M}=-0.293, \mathrm{SEM}=0.172), \mathrm{t}=3.713, \mathrm{p} \leq .001$. Post hoc analysis revealed LPS significantly increased IL-1 $\beta$ transcript $(M=3.324$, SEM $=0.172)$ compared to VEH $(\mathrm{M}=0.249$, SEM $=0.529), \mathrm{t}=4.207, \mathrm{p} \leq .001$. Post hoc analysis revealed LPS significantly increased IL-6 transcript $(M=-1.129, S E M=0.128)$ compared to VEH $(\mathrm{M}=-0.161$, SEM $=0.134), t=5.215, \mathrm{p} \leq .001$ (Fig. 3.3a, $\mathrm{c}, \mathrm{f})$. These data indicate LPS increased neuroinflammation in the dentate gyrus. 


\section{1 hour post RT}

A

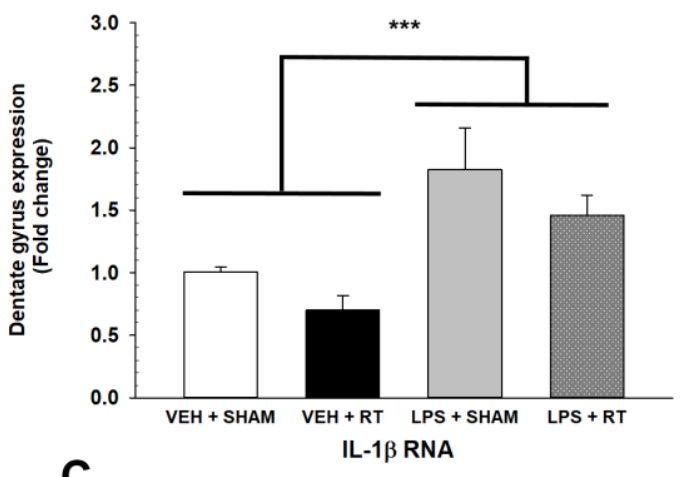

C
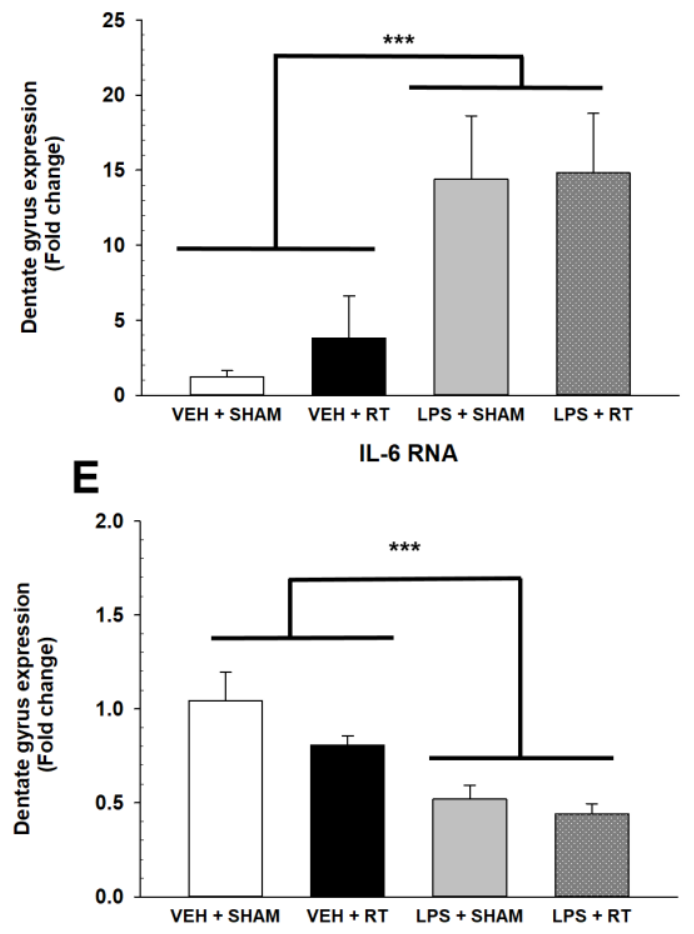

24 hour post RT

TNF- $\alpha$ RNA

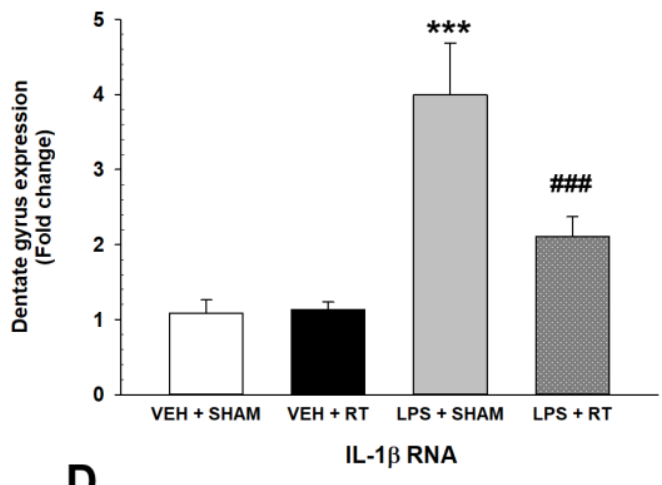

D

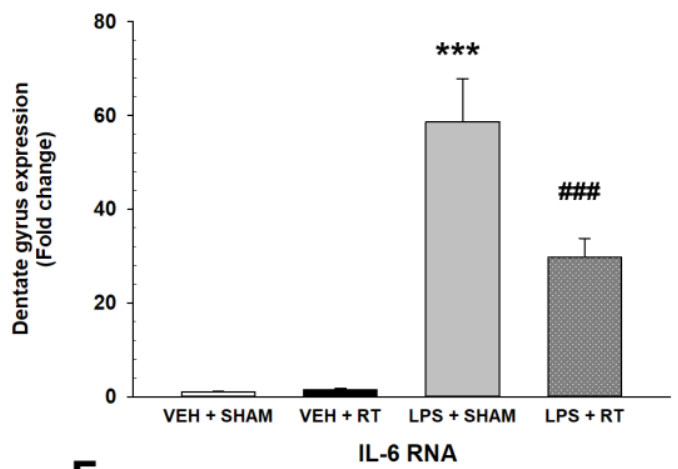

$\mathbf{F}$

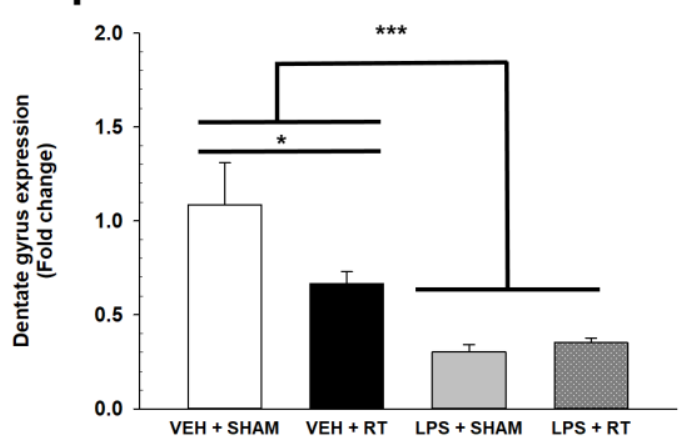

Figure 3.3 LPS-induced markers of neuroinflammation are ameliorated by RT. A \& B) Fold change of proinflammatory transcript TNF- $\alpha$ in the dentate gyrus one and 24-hours post RT. C \& D) Fold change of proinflammatory transcript IL-1 $\beta$ in the dentate gyrus one and 24-hours post RT. E \& F) Fold change of proinflammatory transcript IL-6 in the dentate gyrus one and 24-hours post RT. ${ }^{*}$ denotes a significant effect between groups $(p \leq .05)$, 
${ }^{* * *}$ denotes a significant effect between groups ( $\left.p \leq .001\right)$, and \#\#\# denotes the reversal of a significant effect ( $p \leq .001$ ). Two-way ANOVA was used for comparison between groups $(n=5-6)$. Holm Sidak was used post hoc for multiple comparison procedure. RNA is expressed as fold change normalized to VEH + SHAM. 
Analysis of inflammatory markers at 24-hours post RT revealed a significant interaction between LPS and RT for inflammatory transcripts TNF- $\alpha(F(1,19)=5.354, p=.032)$, IL$1 \beta(F(1,19)=10.589, p=.004)$, and IL-6 $(F(1,19)=5.956, p=.025)$. Post hoc analysis revealed a significant increase in TNF- $\alpha$ transcript in LPS + SHAM (M $=1.894$, SEM $=$ 0.238) compared to VEH + SHAM (M = 0.000, SEM =0.217), $\mathrm{t}=5.885, \mathrm{p} \leq .001 ; \mathrm{a}$ significant increase in TNF- $\alpha$ in LPS + RT $(M=1.017$, SEM $=0.217)$ compared to VEH + $\mathrm{RT}(\mathrm{M}=0.152, \mathrm{SEM}=0.217), \mathrm{t}=2.819, \mathrm{p}=0.011$; and a significant decrease in TNF- $\alpha$ in LPS + RT $(M=1.017$, SEM $=0.217)$ compared to $L P S+S H A M(M=1.894$, SEM $=$ 0.238), $t=2.726, p=.013$. Post hoc analysis revealed a significant increase in IL-1 $\beta$ transcript in LPS + SHAM $(M=5.784$, SEM $=0.231)$ compared to VEH + SHAM $(M=$ $0.000, S E M=0.211), t=18.457, p \leq .001$; a significant increase in IL-1 $\beta$ in LPS + RT (M $=4.832, \mathrm{SEM}=0.211)$ compared to $\mathrm{VEH}+\mathrm{RT}(\mathrm{M}=0.457, \mathrm{SEM}=0.211), \mathrm{t}=14.642, \mathrm{p} \leq$ .001 ; and a significant decrease in IL-1 $\beta$ in LPS + RT $(M=4.832, S E M=0.211)$ compared to LPS + SHAM $(M=5.784$, SEM $=0.231), t=3.039, p=.007$. Post hoc analysis revealed a significant decrease in IL-6 transcript in LPS + SHAM $(M=-1.766$, SEM $=0.186)$ compared to $\mathrm{VEH}+\mathrm{SHAM}(\mathrm{M}=0.000, \mathrm{SEM}=0.170), \mathrm{t}=6.997, \mathrm{p} \leq .001$; a significant decrease in IL-6 in LPS + RT (M = -1.533, SEM = 0.170) compared to VEH + RT $(M=-$ $0.618, \mathrm{SEM}=0.170), \mathrm{t}=3.802, \mathrm{p}=.001$; and a significant decrease in IL-6 in VEH + RT $(\mathrm{M}=-0.618, \mathrm{SEM}=0.170)$ compared to $\mathrm{VEH}+\mathrm{SHAM}(\mathrm{M}=0.000, \mathrm{SEM}=0.170), \mathrm{t}=$ 2.570, $p=.019$ (Fig. 3.3b, d, f). Combined, these data indicate RT ameliorated LPSinduced neuroinflammation in the dentate gyrus.

To further assess the effect of RT on neuroinflammation, a two-way ANOVA was used to examine the effect of LPS and RT on GFAP (at the transcriptional and protein level) in the dentate gyrus at one and 24-hours post RT. Analysis of GFAP transcript at 24-hours revealed a main effect of LPS $(F(1,19)=6.887, p \leq .017)$. Post hoc analysis revealed LPS 
significantly increased GFAP transcript $(M=0.343$, SEM $=0.110)$ compared to VEH $(M=$ $-0.0542, \mathrm{SEM}=0.110), \mathrm{t}=2.624, \mathrm{p}=.017$ (Fig. 3.4c).

Two-way ANOVA analysis of GFAP at the protein level 1-hour post exercise revealed a main effect of LPS $(F(1,20)=4.633, p \leq .044)$. Post hoc analysis revealed LPS significantly increased GFAP protein $(\mathrm{M}=2.425$, SEM $=0.366)$ compared to VEH ( $\mathrm{M}=$ $1.310, \mathrm{SEM}=0.366), \mathrm{t}=2.152, \mathrm{p}=.044$. Two-way ANOVA analysis of GFAP at the protein level 24-hours post exercise revealed a significant interaction between LPS and RT ( $F(1$, $19)=4.689, p \leq .043)$. Post hoc analysis revealed LPS + SHAM had a significantly increased GFAP protein level $(M=1.441$, SEM $=0.117)$ compared to VEH + SHAM $(M=$ 1.000, SEM $=0.107, t=2.785, p=.012 ;$ and LPS $+R T$ had a significantly decreased GFAP protein level $(M=0.961$, SEM $=0.107)$ compared to $L P S+S H A M(M=4.441$, SEM $=0.117$ ), $t=3.031, p=.007$ (Fig. 3.4 b \& d). Combined, these data indicate RT ameliorated reactive astrocyte remodeling induced by LPS. 

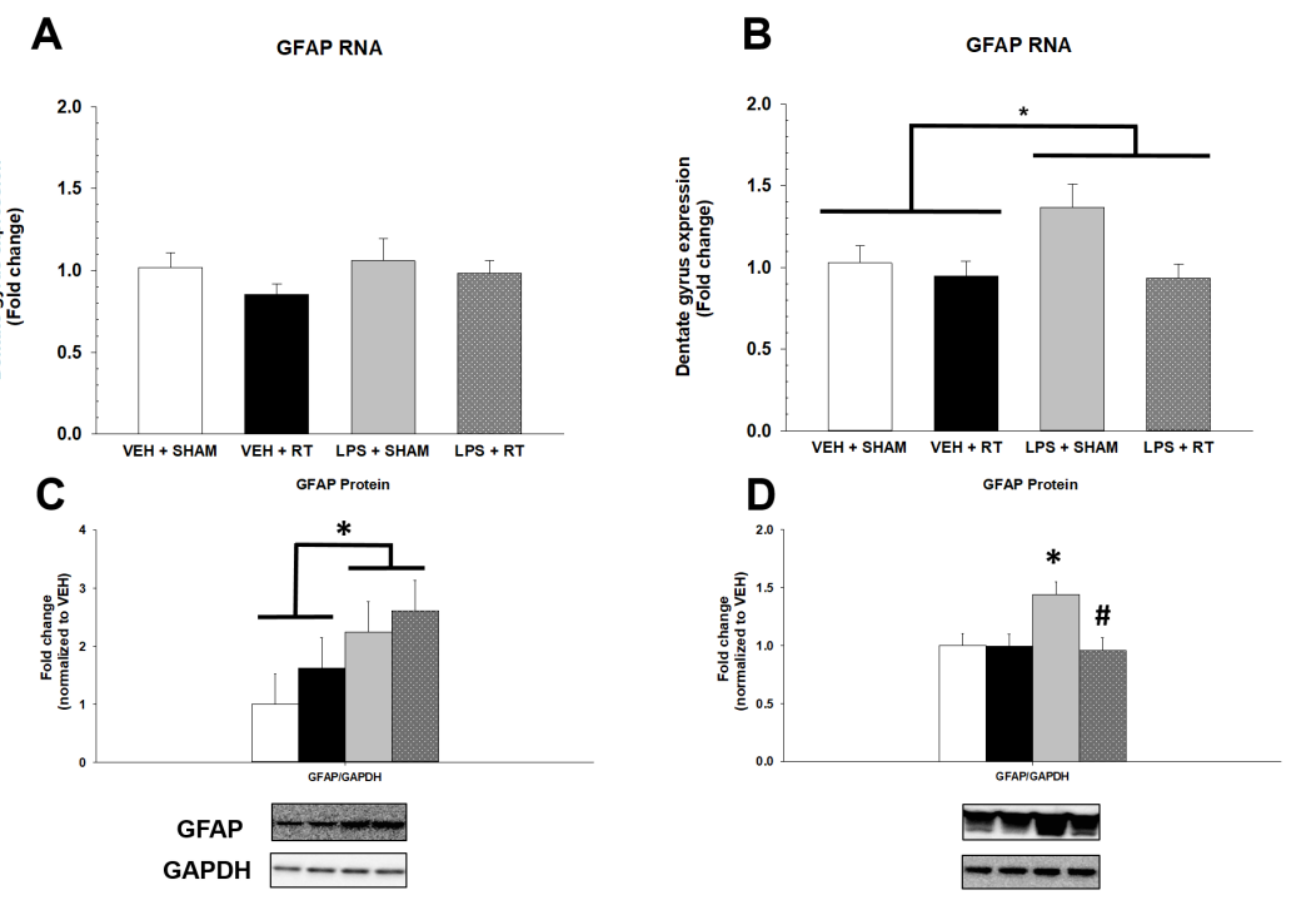

Figure 3.4 LPS-induced reactive astrocyte remodeling was ameliorated by RT. A \& B) Fold change of GFAP transcript in the dentate gyrus one and 24-hours post RT. C \& D) Fold change in GFAP protein in the dentate gyrus one and 24-hours post RT. * denotes a significant effect between groups $(p \leq .05)$. \# denotes the reversal of a significant effect ( $p$ $\leq .05)$. Two-way ANOVA was used for comparison between groups $(n=5-6)$. Holm Sidak was used post hoc for multiple comparison procedure. All RNA and protein are expressed as fold changes and were normalized to VEH + SHAM. 
$R T$ and LPS alter IGF-1 signaling in the dentate gyrus

To assess the effect of RT and LPS on IGF-1 signaling, a two-way ANOVA was used to examine the effect of phosphorylation of proteins concurrent with IGF-1 signaling pathways in the dentate gyrus at one and 24 -hours post RT. Analysis of IGF-1 signaling phosphoproteins at 1-hour post RT revealed a main effect of LPS on IGF-1R phosphorylation $(F(1,20)=6.053, p=.023)$; a main effect of $R T$ on AKT phosphorylation $(F(1,20)=4.379, p=.049)$, a main effect of LPS on ERK phosphorylation $(F(1,20)=$ $5.694, p=.027$ ), and a significant interaction of LPS and RT on the ratio of phosphorylation of GSK to total GSK $(F(1,20)=5.154, p=.034)$. Post hoc analysis revealed LPS significantly increased IGF-1R phosphorylation $(M=1.128$, SEM $=0.0590)$ compared to VEH $(M=0.922$, SEM $=0.0590), t=2.460, p=.023$. Post hoc analysis revealed RT significantly decreased AKT phosphorylation $(M=0.611$, SEM $=0.0993)$ compared to SHAM $(M=0.905$, SEM $=0.0993), t=2.093, p=0.049$. Post hoc analysis revealed LPS significantly increased ERK phosphorylation $(M=1.322, S E M=0.0110)$ compared to SHAM ( $M=0.949$, SEM $=0.0110), t=2.386, p=0.027$ (Fig. 3.5a). Post hoc analysis revealed LPS + SHAM had a significantly decreased phosphorylation GSK $(M=0.418$, $\mathrm{SEM}=0.145)$ compared to VEH + SHAM $(M=1.000, \operatorname{SEM}=0.145, \mathrm{t}=2.843, \mathrm{p}=.010$; and LPS + RT had a significantly increased phosphorylation of GSK $(\mathrm{M}=.887$, SEM $=$ $0.145)$ compared to LPS + SHAM $(M=0.418$, SEM $=0.145), t=2.291, p=.033$ (Fig. 3.5e). Analysis of IGF-1 signaling phosphoproteins at 24 -hour post RT revealed a main effect of LPS on total IGF-1R $(F(1,19)=4.433, p=.049)$. Post hoc analysis revealed RT significantly increased total IGF-1R $(M=1.161, \mathrm{SEM}=0.0444)$ compared to SHAM $(\mathrm{M}=$ 0.1.026, SEM $=0.0465), t=2.105, p=0.049$ (Fig. 3.5d). Combined, these data indicate LPS increased IGF-1R and ERK signaling and RT reduced AKT signaling and restored GSK signaling at 1-hour post RT. 
1 hour post RT
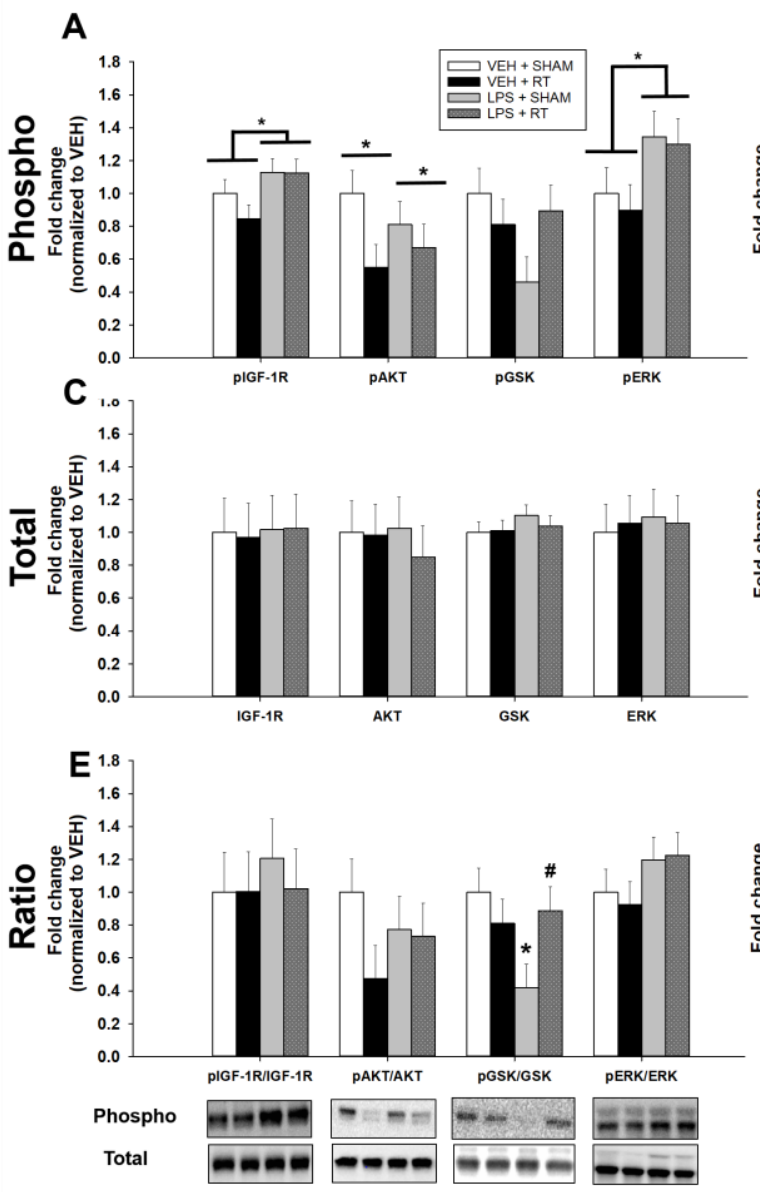

24 hour post RT
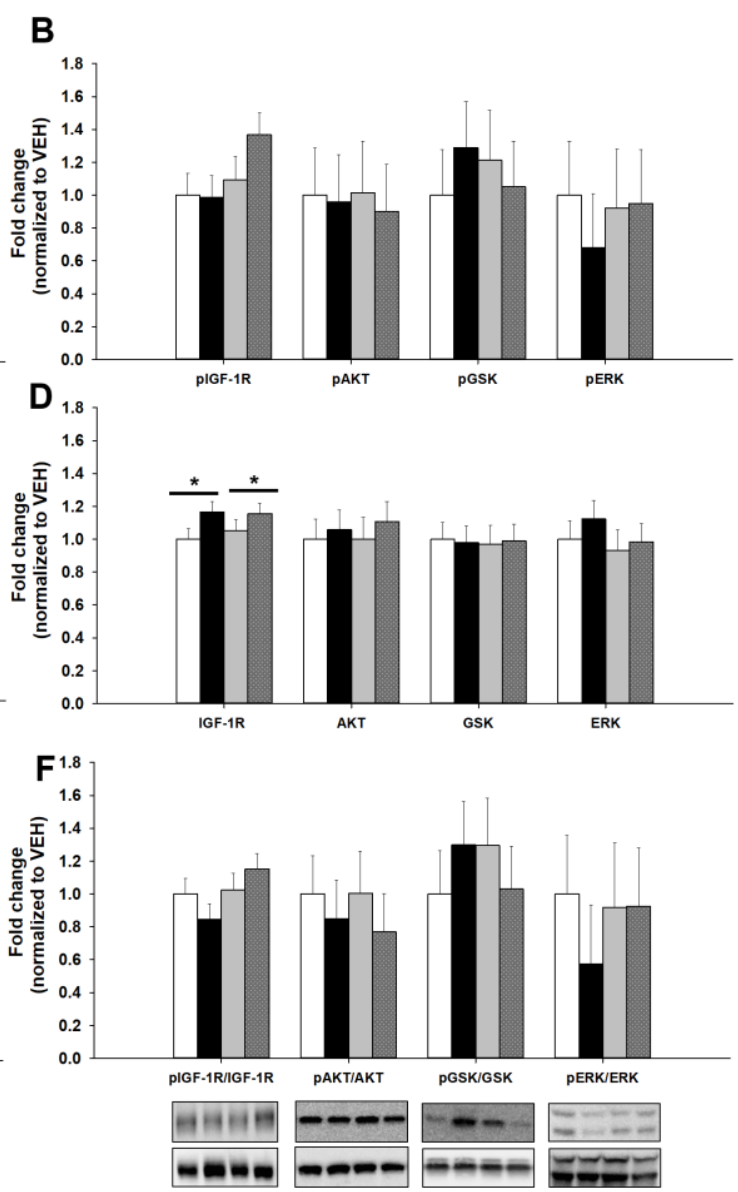

Figure 3.5 Altered dentate gyrus signaling from RT and LPS. A \& B) Phosphoproteins at one and 24-hours, respectively. C \& D) Total proteins at one and 24-hours, respectively. E \& F) Ratio of phosphoproteins to total proteins at one and 24-hours, respectively. * denotes a significant effect between groups ( $p \leq .05)$. \# denotes the reversal of a significant effect $(p \leq .05)$ Two-way ANOVA was used for comparison between groups $(n=$ 5-6). Holm Sidak was used post hoc for multiple comparison procedure. Protein is expressed as fold change normalized to VEH + SHAM. 
To further assess the effect of RT and LPS on IGF-1 signaling in the dentate gyrus, a twoway ANOVA was used to examine transcriptional changes in IGF-1 and IGF-1R one and 24-hours post RT. Analysis of IGF-1R transcript at 1-hour post RT revealed a main effect of both LPS $(\mathrm{F}(1,19)=6.264, \mathrm{p}=.022)$ and $\mathrm{RT}(\mathrm{F}(1,19)=4.442, \mathrm{p}=.049)$. Post hoc analysis revealed LPS significantly decreased IGF-1R transcript $(\mathrm{M}=-0.303$, SEM $=$ $0.0611)$ compared to VEH $(M=-0.0817$, SEM $=0.0641), t=2.503, p=0.022$ (Fig. 3.6a). Post hoc analysis revealed RT significantly decreased IGF-1R transcript $(\mathrm{M}=-0.286, \mathrm{SEM}$ $=0.0611)$ compared to SHAM $(M=-0.0992$, SEM $=0.0641), t=2.108, p=0.049$ (Fig. 3.6c). Analysis of IGF-1 transcript at 1 -hour post RT revealed a main effect of $R T(F(1,19)$ $=4.580, \mathrm{p}=.046)$. Post hoc analysis revealed RT significantly decreased IGF-1R transcript $(M=-0.515$, SEM $=0.0989)$ compared to SHAM $(M=-0.208$, SEM $=0.104), t$ $=2.140, p=0.046$ (Fig. 3.6c). Combined, these data indicate RT decreased dentate gyrus levels of IGF-1, and IGF-1R 1-hour post RT, while LPS decreased dentate gyrus levels of solely IGF-1R. 


\section{1 hour post RT}

IGF-1 RNA

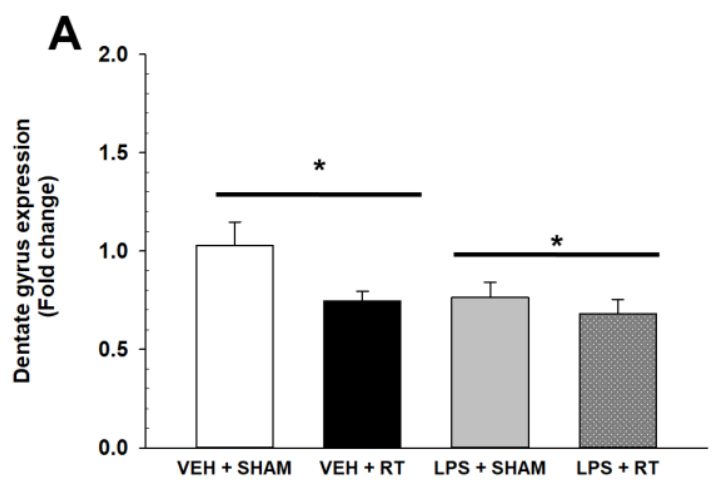

IGF-1R RNA

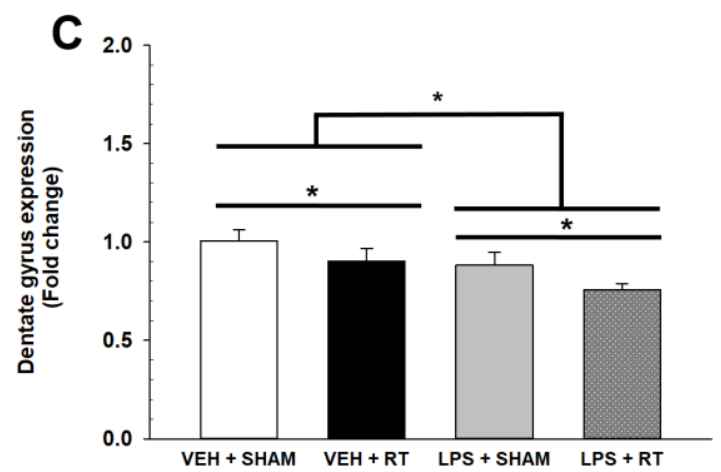

24 hour post RT

IGF-1 RNA

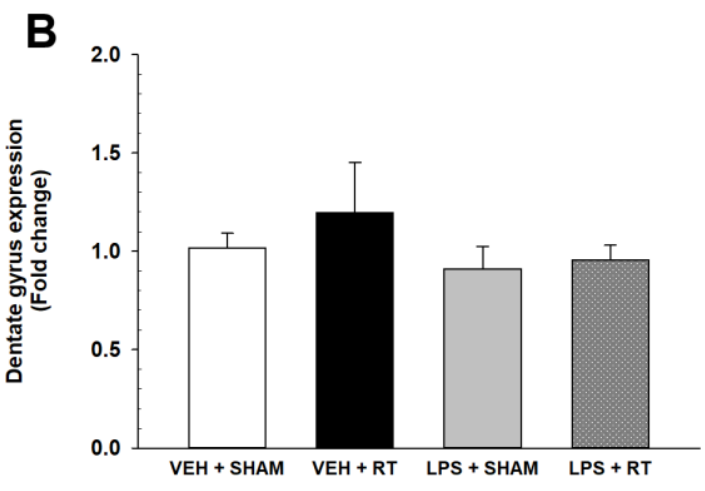

IGF-1R RNA

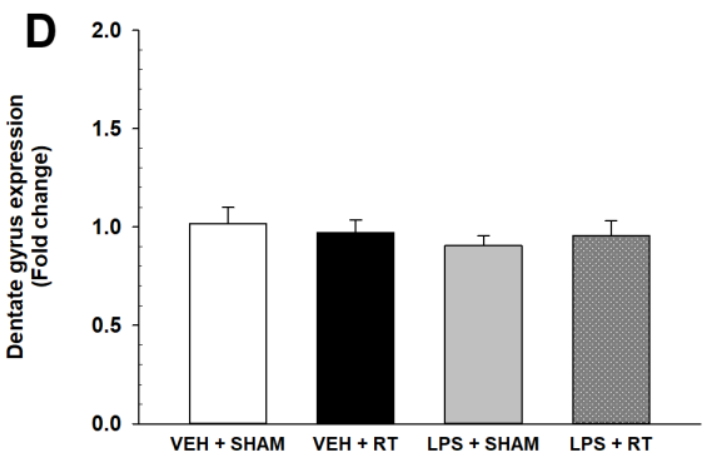

Figure 3.6 RT downregulates IGF-1 and IGF-1R transcription in the dentate gyrus one hour post RT. A \& B) IGF-1R RNA at one and 24-hours, respectively. C \& D) IGF-1 RNA at one and 24-hours, respectively. ${ }^{*}$ denotes a significant effect between groups $(p \leq .05)$. Two-way ANOVA was used for comparison between groups $(n=5-6)$. Holm Sidak was used post hoc for multiple comparison procedure. RNA is expressed as fold change normalized to VEH + SHAM. 


\section{Discussion}

Clinical studies have proposed RT as a suitable intervention to mitigate $\mathrm{MCl}$ (170). Here, we show that RT improves cognitive flexibility, regardless of LPS-induced neuroinflammation. Additionally, we found RT to ameliorate LPS-induced neuroinflammation. Others have reported that RT has a beneficial cognitive effect and inhibits neuroinflammation in the triple-transgenic mouse model of AD (102). Our current data extend these findings, showing that RT can improve multiple forms of memory and that $\mathrm{RT}$ reduces neuroinflammation in a delayed time-dependent manner. Our data also indicate LPS activated IGF-1 signaling is associated with LPS-induced neuroinflammation and that RT regulates IGF-1 locally in the dentate gyrus.

\section{Differential effect of LPS and RT on cognition}

LPS and RT differentially effect novel object recognition, spatial memory, and cognitive flexibility. Here, we found three weeks of RT ameliorated LPS-induced deficits in novel object recognition. Consistent with our findings, Liu et al. reported amelioration of object recognition deficits in triple-transgenic Alzheimer's disease-like mice after RT intervention (ladder climbing) (102). Considering, the dentate gyrus is highly involved in object recognition memory measured during novel object recognition testing (179), and that lentivirus-mediated IL-1 $1 \beta$ knockdown in the hippocampus alleviates LPS-induced object recognition deficits (180), the improved object recognition memory could be partially explained by the reduced neuroinflammation we observed in the dentate gyrus after three weeks of RT.

RT has been reported to improve spatial memory in Morris water maze testing (109). However, in the present study we did not observe changes in spatial memory from RT. This could be due to the shorter duration of RT in this protocol. When RT has been reported to improve spatial memory, rats underwent eight weeks of RT, as opposed to 
only three weeks of RT in our study. We did observe a deficit in spatial memory from LPS, which is consistent with the literature (78).

Cognitive flexibility is defined as the ability to change behavior quickly in the face of changing circumstances and is closely associated with reversal testing in rodents (181, 182). Our current study revealed the number of errors during reversal testing was decreased with three weeks RT, suggesting RT improved cognitive flexibility. Only one other study has examined cognitive flexibility after RT in which Segabinazi et al. reported no change (183). One possible reason for the discrepancy could be due to the different type of test performed (hole board vs reversal testing) to measure cognitive flexibility. Although our data are the first, to our knowledge, to indicate a role for RT to improve cognitive flexibility in a preclinical model, a meta-analysis of healthy adults supports our results showing increases in cognitive flexibility after a single RT workout (184).

\section{RT ameliorates LPS-induced neuroinflammation in a time-dependent manner}

Our data revealed new insights on the effect of RT on neuroinflammation. A reduction in proinflammatory transcripts was observed in dentate gyrus 24-hours post RT. However, there was no amelioration of proinflammatory transcripts at 1-hour post RT suggestive of a phasic time-dependent amelioration of LPS-induced neuroinflammation. Additionally, during three weeks of RT, we also observed a time-dependent decrease in GFAP protein 24-hours post RT. Although GFAP is not an absolute marker of astrocyte reactivity, the amelioration of GFAP observed is indicative of reduced reactive astrocyte remodeling after RT and, therefore, could be an indicator reduced neuroinflammation (185). Liu et al. has found similar results, reporting amelioration of both activated microglia and reactive astrocytes 24-hours post-RT after four weeks of RT (102). 


\section{LPS increases IGF-1 signaling in the dentate gyrus}

We observed an LPS-dependent increase in IGF-1R and downstream ERK phosphorylation at the 1-hour time point. However, there was no observed increase in IGF-1 signaling at the 24-hour time point. One possible reason for the time specific effect could be due to the novelty of the ladder. Novel environment exploration can increase IGF-1 signaling $(186,187)$. Therefore, we postulate that LPS increases sensitivity to IGF-

1 compared to $\mathrm{VEH}$, where exposure to the ladder (for RT or SHAM training) could possibly lead to an increase in IGF-1 signaling at 1-hour and not 24-hours. Although we observed an increase in IGF-1 signaling, we do not know if the increase facilitates the neuroinflammation or is a compensatory defense mechanism to restore the brain to homeostasis. Based on the literature regarding the beneficial effects of IGF-1 (120), we postulate the increased IGF-1 signaling could elicit a neuroprotective effect. This is consistent with the hypothesis that IGF-1 signaling is overactive before becoming resistant during the neurodegenerative process (188), similar to the rise and fall of insulin during type II diabetes progression (189). However, more studies are necessary to determine the role of IGF-1 signaling during neuroinflammation.

\section{$R T$ regulates IGF-1 and IGF-1R in the dentate gyrus}

Our lab and others have reported RT increases IGF-1 signaling in the hippocampus (109, 172). Therefore, we expected an increase in IGF-1R phosphorylation from three weeks of RT. However, RT did not alter IGF-1R phosphorylation at one or 24-hours post RT. RT and LPS transiently reduced IGF-1R RNA in the dentate gyrus 1-hour post RT. Additionally, RT reduced IGF-1 RNA in the dentate gyrus 1 -hour post RT along with an $\mathrm{RT}$-induced decrease in AKT phosphorylation 1-hour post $\mathrm{RT}$ and an increase in IGF-1R protein 24-hours post RT. Studies indicate IGF-1 regulates the IGF-1R, where exogenous IGF-1 has been shown to reduce IGF-1 and IGF-1R mRNA in all brain cell types in cell 
culture. Additionally, an exogenous bolus of IGF-1 decreased brain serum IGF-1 one hour after administration and increased IGF-1 and IGF-1R mRNA after six hours (187). Furthermore, a negative feedback loop that IGF-1/AKT signaling has on its own receptor has been reported (190). These studies are consistent with our own data and indicate a possible bolus of IGF-1 following RT, at a time point not measure during our study, could account for the IGF-1 changes we observe at the RNA and protein level.

LPS and RT differentially effect GSK3 $\beta$ activity in the dentate gyrus Increased GSK3 $\beta$ activity is closely associated with LPS-induced neuroinflammation and cognitive dysfunction $(191,192)$. Phosphorylation of the serine nine residue reduces GSK3 $\beta$ activity possibly providing a therapeutic target to treat neuroinflammation (193). Here we show RT ameliorated LPS-induced deficits in GSK3 $\beta$ phosphorylation. Although the signals that altered GSK3 $\beta$ activity remain ambiguous, increased phosphorylation of GSK3 $\beta$ from RT is consistent with the literature (102). These data indicate that RT could have ameliorated LPS-induced neuroinflammation and $\mathrm{MCl}$ by increasing phosphorylation of serine nine on GSK3ß.

\section{Conclusion}

Here, we show three weeks of RT is able to alleviate LPS-induced neuroinflammation and $\mathrm{MCl}$. This alleviation in neuroinflammation and $\mathrm{MCl}$ is accompanied by reduced GSK3 $\beta$ activity. Additionally, we show for the first time that RT can improve cognitive flexibility regardless of LPS-induced neuroinflammation. We also found RT regulated dentate gyrus IGF-1 and IGF-1R and that LPS increased IGF-1 signaling in dentate gyrus. This data indicate RT may be a powerful intervention to treat neuroinflammation and $\mathrm{MCl}$. 


\section{Chapter Four: Effects of resistance-exercise training and IGF-1 inhibition on cognitive performance in the ICV-LPS neuroinflammatory rat model}

Taylor J. Kelty", Nathan R. Kerra ${ }^{\mathrm{a}}$, Xuansong Mao ${ }^{\mathrm{a}}$, Thomas E. Childs ${ }^{\mathrm{a}}$, and Frank W. Booth ${ }^{\mathrm{a}, \mathrm{b}, \mathrm{c}, \mathrm{d}}$

${ }^{a}$ Department of Biomedical Sciences

${ }^{b}$ Department of Nutrition and Exercise Physiology

${ }^{c}$ Department of Medical Pharmacology and Physiology

${ }^{d}$ Dalton Cardiovascular Research Center

University of Missouri

Columbia, Missouri 65211, U.S.A. 


\section{Abstract}

Resistance-exercise training improves cognition in patients with mild cognitive impairment. However, the underlying mechanisms are still largely unknown. Associative studies, including our own, have suggested IGF-1 signaling as a potential mechanism by which resistance-exercise training improves cognition. Here, we selectively blocked IGF$1 \mathrm{R}$ phosphorylation in the dentate gyrus to test if IGF-1 signaling is required for the cognitive benefits from resistance-exercise training. Female Wistar rats given lipopolysaccharides underwent three weeks of resistance-exercise training or sham training while also receiving picropodophyllin or a vehicle injection immediately before each training workout. We assessed three specific types of cognition (novel object recognition, spatial memory, and cognitive flexibility) to determine the cognitive impact of IGF-1R inhibition during resistance-exercise training. Inhibition of IGF-1R phosphorylation with picropodophyllin in the dentate gyrus during the first resistance-exercise training workout attenuated recognition memory, which disappeared after three weeks. Resistance-exercise training improved spatial memory and cognitive flexibility, regardless IGF-1R inhibition, after three weeks of RT. Additionally, we found IGF-1R inhibition improved cognitive flexibility. Finally, these studies revealed IGF-1R inhibition increased markers of neuroinflammation, which were ameliorated by resistance-exercise training. Collectively, we found resistance-exercise training ameliorated cognitive deficits, despite IGF-1R inhibition. In addition we found IGF-1R inhibition improves cognitive flexibility, independent of resistance-exercise training. 


\section{Introduction}

Mild cognitive impairment $(\mathrm{MCl})$ is considered a transitional state between normalcy and dementia. Patients with $\mathrm{MCl}$ have a six times higher likelihood of developing neurodegenerative dementia (194). Given the prevalence of diagnosed neurodegenerative dementia is predicted to increase to 75 million worldwide by 2030 (165), it is imperative to develop new therapies to treat patients with $\mathrm{MCl}$ to lower the risk of developing neurodegenerative dementia (195). Exercise intervention seems to improve outcomes of patients with $\mathrm{MCl}$, while having little or no benefit for those with advanced stages of neurodegenerative dementia (196). This effect is likely due to the irreversible nature of neurodegenerative dementia (1), supporting early intervention to treat neurodegeneration. Although multiple modalities of exercise exist, the two most common, endurance and resistance-exercise training (RT) both demonstrate cognitive benefits (197). Involved mechanistic studies have revealed both enhanced neurogenesis and brain-derived neurotrophic factor are required to mimic the therapeutic effects of aerobic exercise (116). However, relatively few studies have examined mechanisms by which RT improves cognition, which could be important for the development of new therapies for $\mathrm{MCl}$.

Associative studies, including our own, have suggested IGF-1 signaling as a potential mechanism by which RT improves cognition $(104,109,172)$. Due the neuroprotective effect of insulin-like growth factor-1 (IGF-1) and to the plethora of clinical literature observing IGF-1 elevation after RT (120, 198-200), it seems plausible that IGF-1 signaling could be required for the cognitive benefits from RT that are observed in clinical studies. In the present study, we used a selective insulin-like growth factor-1 receptor (IGF-1R) inhibitor, picropodophyllin (PPP) (201), to test if IGF-1 signaling is required for the cognitive benefits of RT. IGF-1 signaling and RT preclinical studies have been shown to reduce neuroinflammation $(102,202)$, which is an early detectable pathology present in 
patients with $\mathrm{MCI}(75,76)$. Therefore, to test the effects of RT and IGF-1 signaling in a rodent neuroinflammatory model, female Wistar rats underwent intracerebroventricular (ICV) injections of lipopolysaccharides (LPS), which we have previously reported to cause persistent neuroinflammation and cognitive impairment (172). Rats underwent three weeks of RT or sham training while receiving PPP or a vehicle injection immediately before each training workout. Since RT has been shown to improve multiple types of memory in clinical and preclinical studies $(95,102,109)$, in the present study, we assessed three specific types of cognition (novel object recognition, spatial memory, and cognitive flexibility) to determine the cognitive impact of IGF-1R inhibition during RT.

\section{Materials and Methods}

Animal husbandry

Female Wistar rats used in this study were obtained using breeder Wistar rats, approximately 65-days of age, acquired from Charles River Laboratories (Wilmington, MA). Female Wistar rats were weaned at 21 days of age and were grouped housed until surgery. At five weeks of age, rats underwent surgery (described in "cannulation surgeries"). After surgery, rats were single-housed for the remainder of the experiment. Rats were maintained on a 12-hr:12-hr light/dark cycle at $25^{\circ} \mathrm{C}$ and received food and water (Formulab Diet 5008; Purina, St. Louis, MO) ad libitum for the entire study. Rats were divided into four groups: VEH + SHAM (received vehicle injection and sham RT), $\mathrm{VEH}+\mathrm{RT}$ (received vehicle injection and three weeks RT), PPP + SHAM (received IGF1R inhibitor-PPP injection and sham RT), and PPP + RT (received IGF-1R inhibitor-PPP injection and three weeks RT). All groups received ICV-LPS. Rats were divided equally to

ensure each group had a similar average bodyweight at the start of the study $(n=11-12$ per group). The protocol for this study was approved by University of Missouri Institutional 
Animal Care and Use Committee (IACUC), which operates under the U.S. Guide for the Care and Use of Laboratory Animals. The timeline and groups are shown in Fig. 4.1a. 
A

Groups (All groups receive LPS)

1) $\mathrm{SHAM}+\mathrm{VEH}$

2) $\mathrm{RT}+\mathrm{VEH}$

3) $\mathrm{SHAM}+\mathrm{PPP}$

4) $R T+P P P$

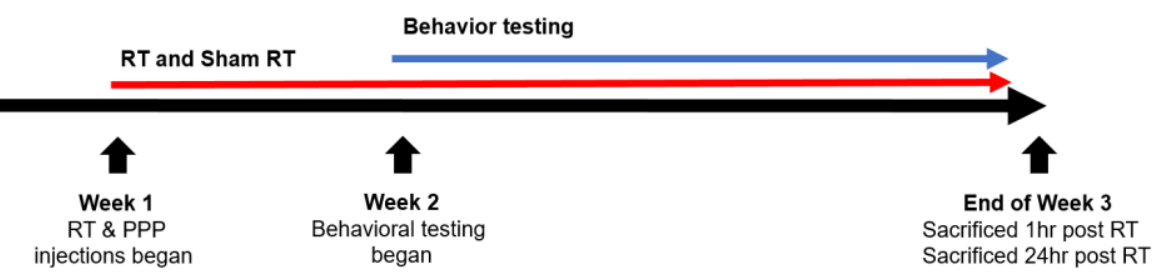

B
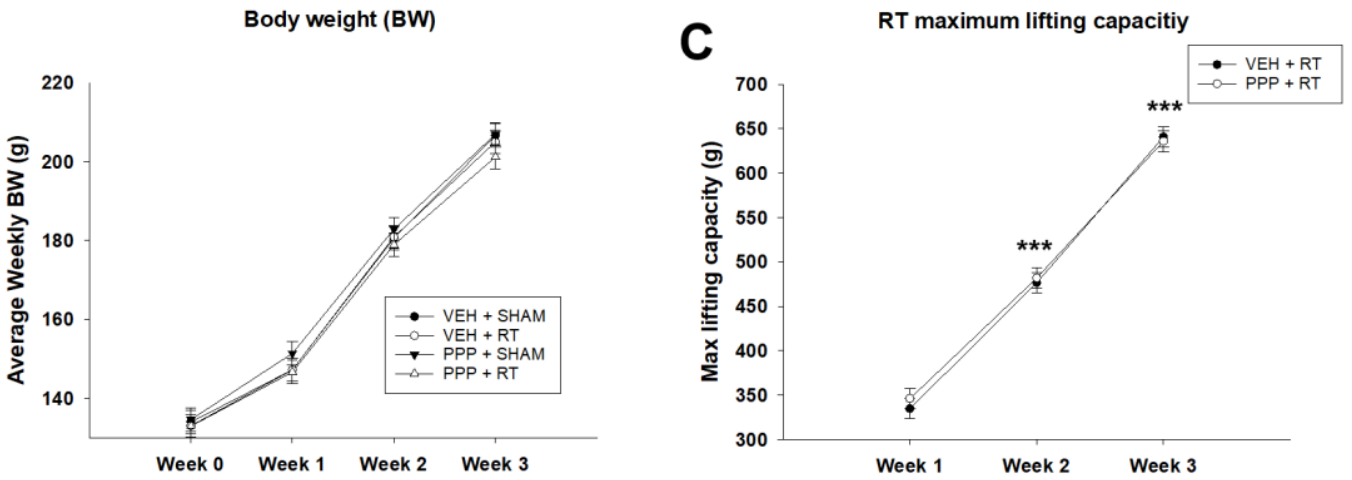

Figure 4.1 Timeline, changes of bodyweight (BW) and lifting capacity during the study. A) Timeline for the study, where all four groups underwent cannulation surgeries, LPS injections and behavioral testing. Groups were divided into four groups: SHAM + VEH, RT + VEH, SHAM + PPP, and RT + PPP then sacrificed at one or 24 hours after the eighth RT workout. B) Average weekly BW for each week of the study. C) Average maximum lifting capacity each week based on the amount of weight a rat could lift in a given week combined with that individual rats BW. ${ }^{* * *}$ denotes a significant effect $(p \leq .001)$. Two-way ANOVA was used for comparison between groups $(n=11-12)$. Holm Sidak was used post hoc for multiple comparison procedure. 


\section{Cannulation surgeries}

In experiment two, rats underwent cannulation surgery where four cannulas were placed in the brain to allow injections of both LPS (bilateral injections into the lateral ventricles) and PPP (bilateral injections into the dentate gyrus). All rats were anesthetized using 2$5 \%$ isoflurane, placed in a stereotactic frame, and prepped for surgery. Rats received banamine at a dosage of $1 \mathrm{ml} / \mathrm{kg}(2.5 \mathrm{mg} / \mathrm{ml})$ as an analgesic. Coordinates for ICV bilateral cannula placement were $0.8 \mathrm{~mm}$ posterior to bregma, $1.5 \mathrm{~mm}$ lateral to the midline suture (on either side), and $2.1 \mathrm{~mm}$ below the skull surface. Coordinates for dentate gyrus bilateral cannula placement were $2.8 \mathrm{~mm}$ anterior to bregma, $1.5 \mathrm{~mm}$ lateral to the midline suture (on either side), and $2.1 \mathrm{~mm}$ below the skull surface. A 28 gage needle (Component One, Pittsburgh, PA), extending two $\mathrm{mm}$ past the cannula was used to bilaterally inject LPS solution (5 $\mu \mathrm{g} / \mu \mathrm{l})$ (Escherichia coli O55:B5, Sigma, St. Louis, MO) at a rate of one $\mu \mathrm{l} / \mathrm{min}$ followed by a 2-minute waiting period for solution to be absorbed in the lateral ventricles. PPP $(0.25 \mu \mathrm{M})$ or VEH (saline with $0.2 \%$ DMSO) solution was infused with a 28 gage needle extending two $\mathrm{mm}$ past the cannula at a rate of $0.334 \mu \mathrm{l} / \mathrm{min}$ followed by a 3minute waiting period for the solution to be absorbed in the dentate gyrus (MilliporeSigma, Burlington, MA) or respective vehicle (saline solution). LPS injections occurred 2-3 days post cannulation surgery. PPP or vehicle injections occurred immediately before each RT workout.

\section{Resistance-exercise training protocol}

RT protocol was similar to previous studies (172). Briefly, rats would have weights in a holding apparatus attached to the base of the tail with athletic tape and undergo eight 100cm climbs with increasing weight. Rats trained three days a week for three weeks (the last week of RT rats had only two workouts before sacrifice, therefore eight RT workouts total). The first day of each week of training was used to determine the maximum lifting capacity 
of each rat followed by two maintenance days based on that individual rat's max lifting capacity with1-2 rest days between each workout. SHAM groups received a sham-RT where rats climbed $27-\mathrm{cm}$ up the ladder using a sham start platform without weights attached to the tail.

\section{Elevated plus maze testing}

The elevated plus maze is used in rodents for measuring anxiogenic behavior based on a rodent's aversion to open spaces (174). The elevated plus maze is a plus shaped platform consisting of four arms. Two of the arms on the platform have walls and are considered "closed" arms and two arms do not have walls and are considered "open" arms. Each arm of the maze is $43-\mathrm{cm}$ long $\times 14-\mathrm{cm}$ wide, closed platforms have $22-\mathrm{cm}$ tall walls on three sides. The entire maze is $63-\mathrm{cm}$ off the ground. All animals were brought into testing room a minimum of 20 minutes before the experiment started to acclimate them to the room. Temperature, light and noise were also controlled for during testing using a thermometer, lux meter, and decibel meter, respectively. Each session began by gently placing the rat at the junction of the open and closed arms. Rat were given five minutes to explore freely where time during in the open arm was recorded. The apparatus was cleaned with ethanol (70\%) between each session to reduce any odor biases. Non-biased AnyMaze behavior tracking software was used to record all data (Stoelting Co., Wood Dale, IL). The time spent in the open arm was analyzed as a measurement of anxiety-like behavior.

\section{Barnes maze and reversal testing}

The Barnes maze test is a cognitive behavioral test to measure spatial learning/memory in rodents based on the rodents' aversion to an open and well-lit areas (175). A rotating circular gray Barnes maze platform (122-cm in diameter) with 20 holes (each being 10-cm in diameter) was used to test spatial learning/memory behavior. Under one of the 20 holes 
was a hidden goal box (30-cm in length $X 15-\mathrm{cm}$ wide $X 13-\mathrm{cm}$ high) for the rat to climb into and escape from the aversive open and well-lit platform. The 76-cm high circular platform rotated, allowing each rat to be assigned a different target hole to escape into, which helped eliminate odor cues when testing. The rotating platform also controlled for any biases from visual cues by keeping the same location of the target hole for each rat. A thermometer, lux meter, and decibel meter were used to monitor temperature, light, and sound, respectively. Testing occurred during the rats' light cycle for five consecutive days (two trials per day). Before the first trial rats are placed in the goal box for two minutes to help associate the goal box as a safe environment. During each trial, the rat was placed in the center of the maze under a start box for 30 seconds, then had five minutes to explore the maze. The rat was allowed 30 seconds to stay in the goal box to associate it as a safe place. Afterward, the rat was taken out of the goal box and was placed back into its home cage. The Barnes maze platform was cleaned with $70 \%$ ethanol to help eliminate odor cues. The time taken to find and step into the whole (latency to goal box) and number of errors (nose pokes into the incorrect hole) was recorded. Design and methods were adapted from previous experiments (175). Non-biased AnyMaze tracking software (Stoelting, Wood Dale, IL) was used to record Barnes maze data. During reversal testing, the hole was placed in a new location $180^{\circ}$ from the original location. The rat underwent reversal testing following the last day of Barnes maze testing. The reversal testing lasted three consecutive days ( 2 trials per day); following a similar protocol to Barnes maze testing.

\section{Novelty object recognition testing}

The novelty-object recognition test is a behavioral test used to measure recognition memory in rodents (176). Rats are placed in a box (60-cm in length X 60-cm width X 46$\mathrm{cm}$ height) and allowed to explore similar and novel objects. The test is based on the 
natural proclivity of a rodent to explore novel objects opposed to familiar ones (203). Testing consisted of three phases' habituation, familiarization, and novelty object recognition testing. During habituation, rats were given five minutes to explore the box without any objects to acclimate them to the new environment. During familiarization, rats are given up to ten minutes to explore the box with two identical objects sitting $5-\mathrm{cm}$ from the wall on each side. Familiarization is performed 24 hours after habituation phase. During novel object recognition testing, a novel object replaces one of the similar objects from familiarization and rats are given ten minutes to explore. Novel object recognition testing takes place approximately one hour after the familiarization phase. The experiment was stopped after the rat had explored the objects for a total of 20 seconds or when the 10-minute time period was over (i.e., the maximum session time). The time spent exploring each object was recorded and analyzed (time spend exploring novel object/ time spent exploring both objects in total) to create an object preference. Temperature, light, and sound were monitored during testing and the novelty-object recognition box was cleaned with $70 \%$ ethanol between sessions to help eliminate odor cues. These methods were modified from Leger et al (176).

\section{Tissue Collection}

Tissue collection took place at 1-hour and 24-hours after the last RT or sham workout. Rats were euthanized via carbon dioxide asphyxiation. The brain was extracted and washed in cold saline solution. $2 \times 3 \mathrm{~mm}$ thick dentate gyrus punches taken from coronal brain slices sliced using a brain matrix (Braintree Scientific, Braintree, MA) identified using a rat brain atlas (177). Dentate gyrus punches were flash frozen with liquid nitrogen and stored at $-80^{\circ} \mathrm{C}$ until processing.

RNA Isolation, cDNA Synthesis, and qRT-PCR 
Dentate gyrus RNA isolation, cDNA synthesis, and qRT-PCR were performed as described previously (172). Briefly, dentate gyrus punches were place in TRIzol (Invitrogen, Carlsbad, CA) and homogenized three times for one min at $25 \mathrm{~Hz}$ using RNase-free, stainless steel beads and a Tissuelyser (Qiagen, Germantown, MD). Samples were cooled in ice for one minute after each homogenization to prevent RNA degradation. Using TRIzol protocol, RNA was extracted and quantified using a Nanodrop 1000 (Thermo Scientific, Waltham, MA). Following quantification, cDNA synthesis was completed by treating RNA with DNase using DNase I (Thermo Scientific) and reverse transcribed using a High Capacity cDNA Reverse Transcription Kit (Applied Biosystems, Carlsbad, CA). Gene-specific primers were constructed using Primer3 (Table 4.1) that were used for qRT-PCR. cDNA from each sample was assayed in duplicate for target genes in Table 4.1 using iTaq Universal SYBR green Supermix (Bio-Rad Laboratories, Hercules, CA) and RNA expression values were normalized to $18 \mathrm{~S}$ and quantified by the ${ }^{\Delta \Delta} \mathrm{Ct}$ Method. To avoid statistical analysis on logarithmically transformed data, changes in the $\triangle \mathrm{CT}$ was used for statistical analysis. Graphs are expressed as fold change (normalized to VEH + SHAM). 


\begin{tabular}{|c|c|c|c|}
\hline Gene & Forward $\left(5^{\prime}-3^{\prime}\right)$ & Reverse (5'-3') & Accession number \\
\hline $18 \mathrm{~S}$ & GCTCGCTCCTCTCCTACTTG & GATAAATGCACGCGTTCCCC & NR_046237.2 \\
\hline TNF- $\alpha$ & AACACACGAGACGCTGAAGT & TCCAGTGAGTTCCGAAAGCC & NM_012675.3 \\
\hline IL-1 $\beta$ & TGACTTCACCATGGAACCCG & GACCTGACTTGGCAGAGGAC & NM_031512.2 \\
\hline IL-6 & CAGTTGCCTTCTTGGGACTGA & GAAGTCTCCTCTCCGGACTTG & NM_012589.2 \\
\hline GFAP & TTGACCTGCGACCTTGAGTC & GAGTGCCTCCTCCTGGTAACTCG & NM_017009.2 \\
\hline IGF-1 & CGTCTAGGCCATTAGCCCTG & AGGGGCGTTAAAACTTGGGT & NM_178866.4 \\
\hline IGF-1R & CTGGGTGTAGTATCCCAAGGC & GGACTAGATTATTCTCCACCTCTGG & NM_052807.2 \\
\hline
\end{tabular}

Table 4.1. Primer sequences used for gene expression analysis by qRT-PCR. 


\section{Immunoblotting}

Methods for immunoblotting were similar to previous studies (172). Briefly, protein concentration was obtained using BCA assay (Pierce Biotechnology, Rockford, IL) from dentate gyrus rich plugs isolated for protein. 17.5 micrograms of protein was loaded for electrophoresis and transferred to nitrocellulose membrane. Immunoblots were incubated with Ponceau S (Sigma, St. Louis, MO) to verify equal loading in each lane. After blocking with $5 \%$ milk and washing, blots were bathed in primary antibody overnight at $4^{\circ} \mathrm{C}$. Primary antibodies included: IGF-1 receptor beta (IGF-1Rß) (1:2000) (Cell Signaling, Danvers, MA) and Phospho-IGF-I Receptor $\beta$ (Tyr 1135/1136)/Insulin Receptor $\beta$ (Tyr 1150/1151) (19H7) (1:500) (Cell Signaling, Danvers, MA). Phospho-IGF-1R $\beta$ was applied for three days at $4^{\circ} \mathrm{C}$ before applying secondary antibody. Horseradish peroxidase (HRP)conjugated secondary (1:1000) (Cell Signaling, Danvers, MA) was applied for one hour at room temperature the following day. HRP substrate (MilliporeSigma, Burlington, MA) was applied for five minutes before exposure. A Kodak 4000R Imager with Molecular Imagery Software (Kodak Molecular Imaging Systems, New Haven, CT) was used to determine band densitometry. Molecular weight was estimated using Precision Plus Protein Standards (Bio-rad Laboratories, Hercules, CA).

\section{Statistical Analysis}

All analytical procedures were performed using SigmaPlot 14.0 (Systat Software, San Jose, CA). One-way ANOVA was used for comparison of trials during Barnes maze and reversal testing. Two-way ANOVA was used for analyses. Barnes analysis did not use trial one and reversal trial one during analysis, because during these trials rats had never seen the location of the goal box and, therefore, does not reflect learning/memory. HolmSidak test was applied post hoc for all pairwise multiple comparison procedures. A $p \leq .05$ 
was used as the significance level. Values are expressed as means (M) and standard errors of the mean (SEM).

\section{Results}

Maximum lifting capacity increased each week during $R T$

To verify RT, a two-way ANOVA was used to analyze maximum lifting capacity during the three weeks of ladder climbing. Analysis of maximum lifting capacity revealed a main effect of weeks, $F(2,64)=336.283, p \leq .001$. Post hoc analysis revealed increased lifting capacities for each week. Week two $(M=479.304, S E M=8.221)$ was significantly higher than week one $(M=340.362$, SEM $=8.040), t=12.083, p \leq .001$; and week three $(M=$ $638.469, \mathrm{SEM}=8.221)$ was significantly higher than week two $(\mathrm{M}=479.304, \mathrm{SEM}=$ 8.221), $t=13.691, p \leq .001$ (Fig. 4.1c). These data indicate RT was able to increase maximum lifting capacity during RT protocol.

\section{PPP injection before the first RT workout reduced recognition memory}

To assess recognition memory after the workout, a two-way ANOVA was used to examine the effect of PPP injections and RT after the first RT workout in rats given LPS. Analysis of novel object preference revealed a significant interaction between PPP injections and one RT workout $\mathrm{F}(1,20)=6.861, \mathrm{p}=.025$. Post hoc analysis revealed PPP + RT had a significantly decreased preference to the novel object $(M=0.375$, SEM $=0.0731$ ) compared to VEH + RT ( $M=0.745, S E M=0.0731), t=3.571, p=.002$ (Fig. 4.2b). These data indicate inhibition of IGF-1R during an acute RT workout could impair novel recognition memory.

Three weeks of RT improved recognition memory, independent of PPP injections 
To assess recognition memory after three weeks of RT, a two-way ANOVA was used to examine the effect of PPP injections and RT after three weeks RT in rats given LPS. Analysis of novel object preference revealed a main effect of RT, independent of PPP injections $F(1,43)=10.172, p=.003$. Post hoc analysis revealed three weeks RT significantly increased novel object preference $(M=0.625$, SEM $=0.0254)$ compared to SHAM $(M=0.512, S E M=0.0248), t=3.189, p=.003($ Fig. 4.2c). These data indicate RT improved recognition memory functions independent of IGF-1 signaling.

\section{Verification of learning during Barnes maze and reversal testing}

To verify Barnes maze and reversal testing, a one-way ANOVA was used to examine the effect of trial for latency and number of errors. Analysis revealed a main effect of trial for latency $(F(9,465)=10.189, p \leq .001)$ and errors $(F(9,463)=4.899, p \leq .001)$ made during Barnes maze testing. Post hoc analysis revealed trial ten time $(M=16.319, S E M=3.107)$ and errors $(M=1.872, S E M=0.375)$ were significantly lower than trial one time $(M=$ 47.875, $\mathrm{SEM}=8.049 ; \mathrm{t}=5.127, \mathrm{p} \leq 0.001)$ and errors $(\mathrm{M}=4.583, \mathrm{SEM}=0.696 ; \mathrm{t}=3.695$, $\mathrm{p}=.010)($ Fig. $4.2 \mathrm{~d} \& \mathrm{e})$. Analysis revealed a main effect of trial for latency $(F(5,276)=$ $12.967, p \leq .001)$ and errors $(F(5,276)=11.269, p \leq .001)$ made during reversal testing. Post hoc analysis revealed trial six time $(M=15.362, \mathrm{SEM}=2.112)$ and errors $(M=2.319$, SEM $=0.447)$ were significantly lower than trial one time $(M=44.809, S E M=5.120 ; t=$ 6.642, $p \leq 0.001)$ and errors $(M=6.957$, SEM $=0.900 ; t=6.103, p \leq 0.001)$ (Fig. 4.2h \& i). Combined, these data indicate rats were able to learn Barnes maze testing and reversal testing over time.

\section{$R T$ improved spatial memory, independent of PPP injections}

To assess spatial memory, a two-way ANOVA was used to examine both RT and PPP injection effects on Barnes maze testing in rats given LPS. Analysis of errors revealed a 
main effect of $R T, F(1,421)=4.585, p=.033$. Post hoc analysis revealed three weeks RT significantly reduced errors $(M=2.338, \operatorname{SEM}=0.242)$ compared to SHAM $(M=3.066$ SEM = 0.239), $\mathrm{t}=4.087, \mathrm{p} \leq .001$ (Fig. 4.2g). These data indicate RT improved spatial memory functions, independent of IGF-1 signaling.

$R T$ and PPP injections can improved cognitive flexibility, independent of one another To assess cognitive flexibility, a two-way ANOVA was used to examine both RT and PPP injection effects on reversal testing. Analysis of latency revealed a main effect of PPP injection on reversal testing, $F(1,231)=9.705, p=.002$. Post hoc analysis revealed PPP injection significantly decreased latency $(M=16.091, S E M=1.653)$ compared to VEH (M $=23.292, \mathrm{SEM}=1.616), \mathrm{t}=3.115, \mathrm{p}=.002$ (Fig. 4.2j). Analysis of errors revealed a main effect of $R T(F(1,231)=7.903, p=.005)$ and PPP injections $(F(1,231)=4.499, p=.035)$. Post hoc analysis revealed RT significantly decreased errors $(\mathrm{M}=2.380, \mathrm{SEM}=0.276)$ compared to SHAM $(M=3.467$, SEM $=0.270), t=2.811, p=.005$. Post hoc analysis also revealed PPP injections significantly decreased errors $(\mathrm{M}=2.514, \mathrm{SEM}=0.276)$ compared to SHAM $(M=3.333, \mathrm{SEM}=0.270), \mathrm{t}=2.121, \mathrm{p}=.035$ (Fig. 4.2k). These data indicate both RT and PPP injection separately improved cognitive flexibility.

\section{Anxiogenic behavior did not account for the changes we observed in cognition}

There is an established relationship between anxiety and cognition (178), therefore, we used elevated plus maze to determine if there were any changes in anxiety from our treatments that could have influenced our cognitive behavioral tests. However, we found no change in anxiety-like behavior from RT or LPS (Fig. 4.2a), suggesting anxiety-like behavior did not account for the changes we observed in cognition. 

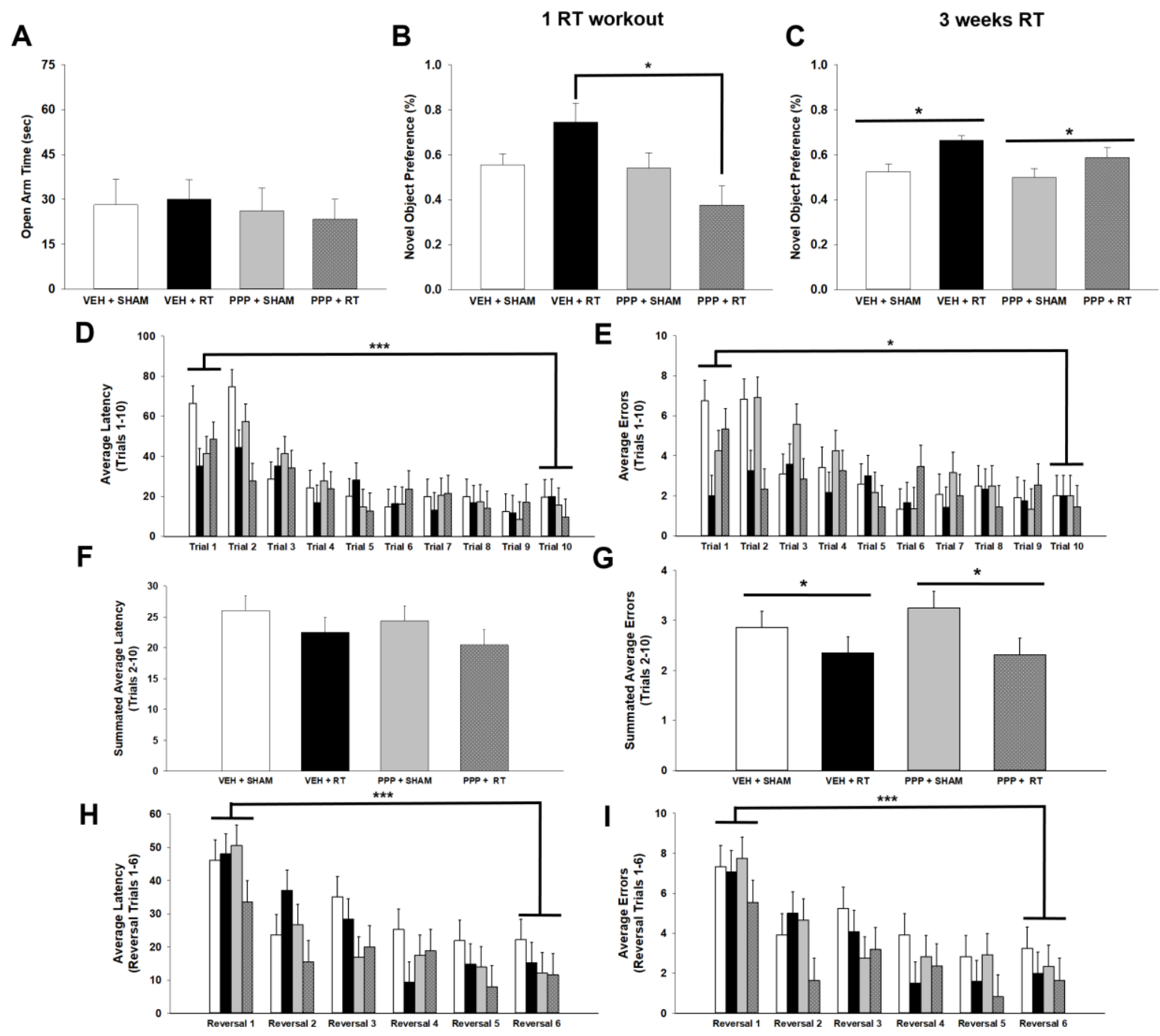

$\mathbf{J}$

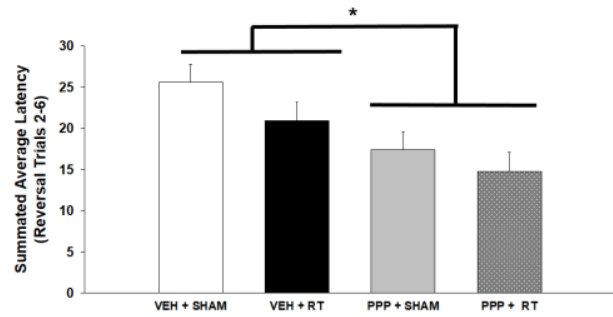

K

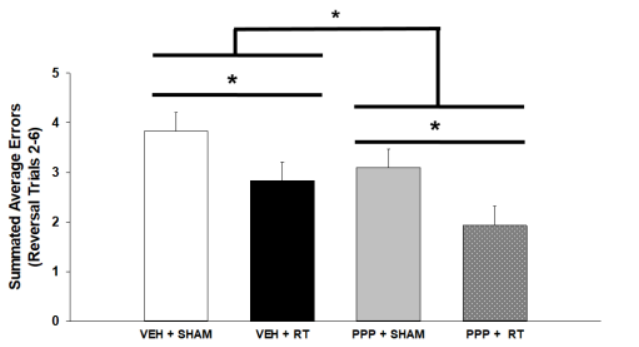

Figure 4.2. Behavioral testing revealed RT and PPP injections had differential effects on object recognition memory, spatial memory, and cognitive flexibility. A) Time spent in open arm of elevated plus maze. B) Preference for novel object during novel object recognition after one RT workout. C) and three weeks of RT. D) Average latency time (seconds) to 
find goal box during five days of Barnes maze testing (two trials/day). E) Average errors made before finding goal box during five days of Barnes maze testing (two trials/day). F) Summated average latency (excluding Trial 1) during Barnes maze testing. G) Summated average errors (excluding Trial 1) during Barnes maze testing. H) Average latency time (seconds) to find goal box during three days of reversal testing (two trials/day). I) Average errors made before finding goal box during three days of reversal testing (two trials/day). J) Summated average latency (excluding Trial 1) during reversal testing. K) Summated average errors (excluding Trial 1) during reversal testing. * denotes a significant effect ( $p$ $\leq .05)$. \# denotes the reversal of a significant effect $(p \leq .05) .{ }^{* * *}$ denotes a significant effect $(p \leq .001)$. For differences between trials, a one-way ANOVA was used for comparison between groups. For all other analyses, a two-way ANOVA was used for comparison between groups $(n=11-12)$. Holm Sidak test was used post hoc for multiple comparison procedure. 
PPP injections decrease IGF-1R phosphorylation in the dentate gyrus

To assess IGF-1 signaling in the dentate gyrus after both RT and PPP injections, a twoway ANOVA was used to examine IGF-1R phosphorylation at one and 24-hours post RT. Analysis of IGF-1R phosphorylation revealed a main effect of PPP injections at one hour $(F(1,20)=4.978, p=.037)$. Post hoc analysis revealed PPP injection significantly decreased IGF-1R phosphorylation $(\mathrm{M}=0.678, \mathrm{SEM}=0.122)$ compared to $\mathrm{VEH}(\mathrm{M}=$ 1.065, SEM $=0.122), t=2.231, p=0.037$ (Fig. 4.3a). These data verified PPP injections inhibited IGF-1R phosphorylation in the dentate gyrus. 
1 hour post RT
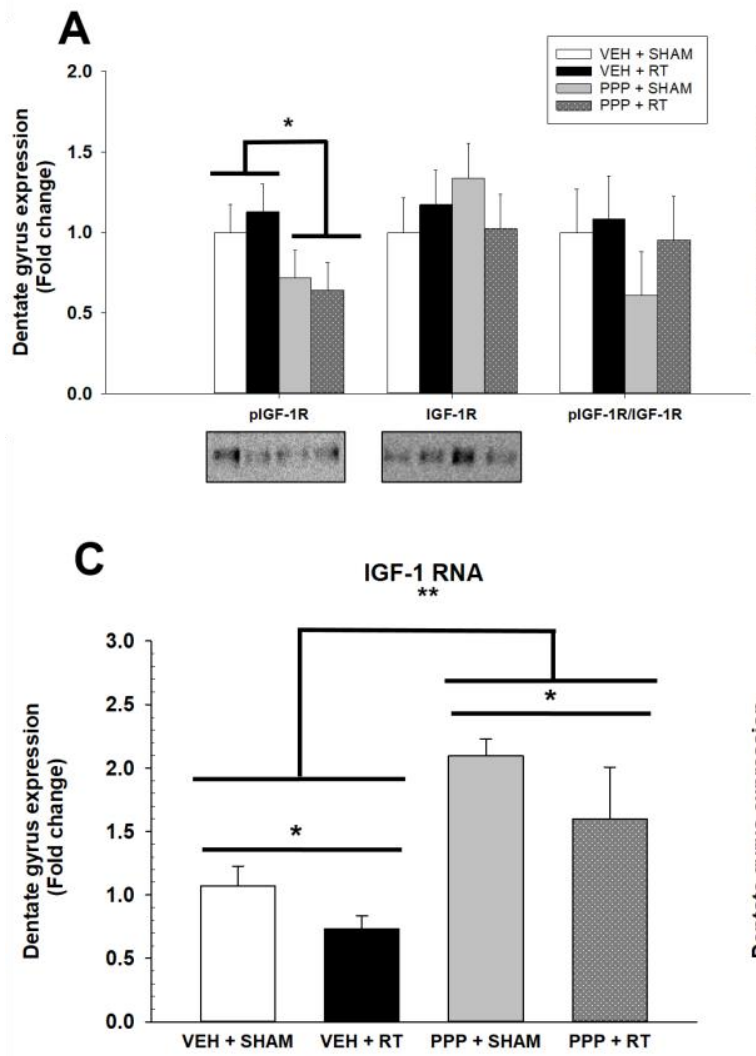

E

IGF-1R RNA

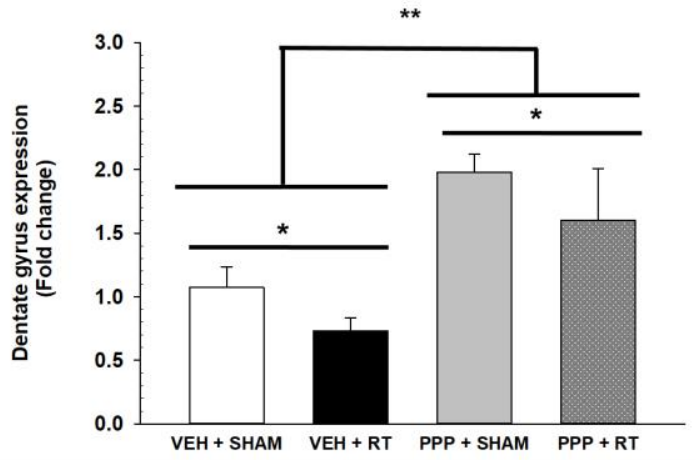

24 hour post RT

B

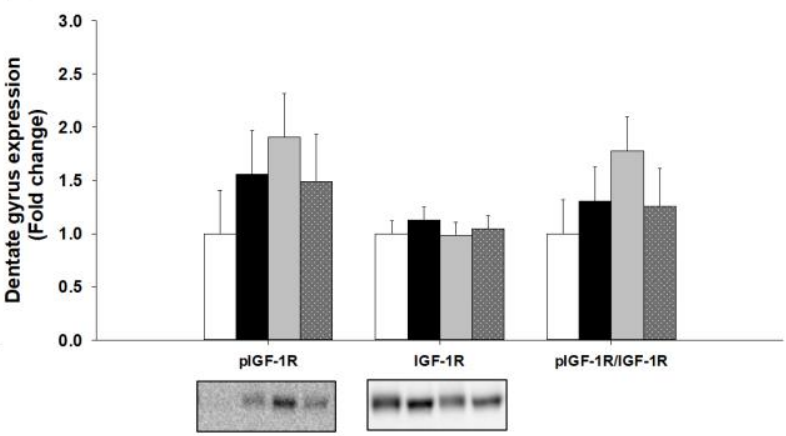

D

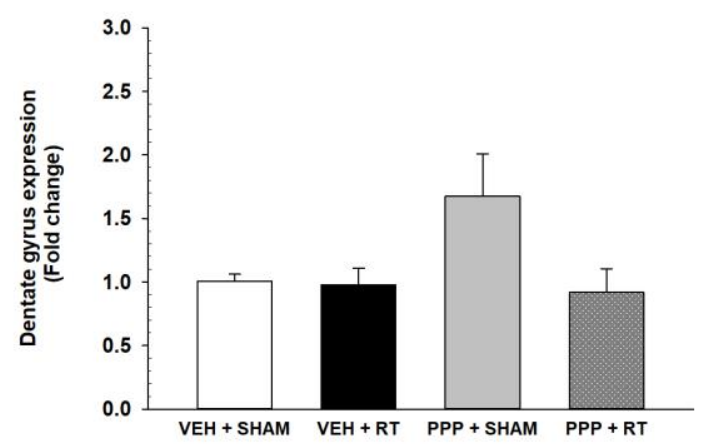

$\mathbf{F}$

IGF-1R RNA

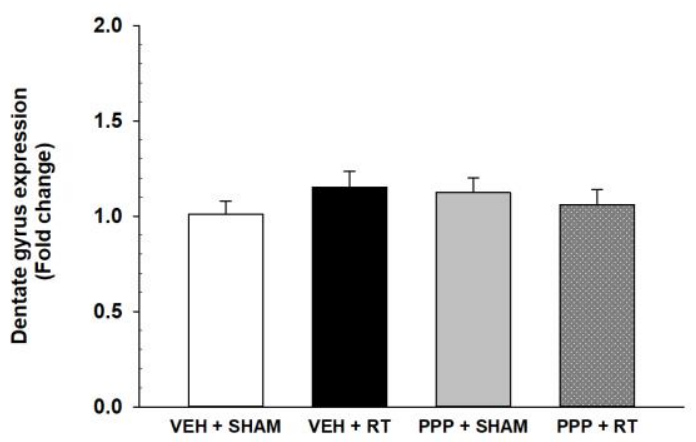

Figure 4.3. RT and PPP injection effects on IGF-1R phosphorylation and RNA levels in the dentate gyrus. A \& B) IGF-1R phosphoprotein, IGF-1R total protein, and ratio at one and 24-hours, respectively. C \& D) IGF-1R RNA at one and 24-hours, respectively. E \& F) IGF-1 RNA at one and 24-hours, respectively. ${ }^{*}$ denotes a significant effect between groups $(p \leq .05) .{ }^{* *}$ denotes a significant effect between groups $(p \leq .01)$. Two-way ANOVA 
was used for comparison between groups $(n=5-6)$. Holm Sidak was used post hoc for multiple comparison procedure. RNA is expressed as fold change normalized to VEH + SHAM. 
$R T$ and IGF-1R inhibition differentially alter IGF-1/IGF-1R transcripts in the dentate gyrus To assess local IGF-1/IGF-1R RNA level, after both RT and PPP injections, a two-way ANOVA was used to examine transcriptional changes in IGF-1 and IGF-1R at one and 24hours post RT. Analysis of IGF-1 RNA at 1-hour post RT revealed a main effect of both $\mathrm{RT}(\mathrm{F}(1,20)=4.640, \mathrm{p}=.044)$ and PPP injection $(F(1,20)=12.630, \mathrm{p}=.002)$. Post hoc analysis revealed RT significantly decreased IGF-1 transcript $(M=-0.0675, S E M=0.195)$ compared to SHAM $(\mathrm{M}=0.527, \mathrm{SEM}=0.195), \mathrm{t}=2.154, \mathrm{p}=0.044$. Post hoc analysis also revealed PPP injection significantly increased IGF-1 transcript $(\mathrm{M}=0.721$, SEM $=$ 0.195) compared to SHAM ( $M=-0.261$, SEM $=0.195), t=3.554, p=0.002$ (Fig. 4.3c). Analysis of IGF-1R RNA at 1-hour post RT revealed a main effect of both $R T(F(1,20)=$ 4.673, $\mathrm{p}=.043)$ and PPP injection $(\mathrm{F}(1,20)=9.475, \mathrm{p}=.006)$. Post hoc analysis revealed RT significantly decreased IGF-1R transcript $(M=-0.130$, SEM $=0.201)$ compared to SHAM $(M=0.483$, SEM $=0.201), t=2.162, p=0.043$. Post hoc analysis also revealed PPP injection significantly increased IGF-1R transcript $(M=0.613$, SEM $=0.201$ ) compared to SHAM $(M=-0.260, \operatorname{SEM}=0.201), t=3.078, p=0.006$ (Fig. 4.3e). Combined these data indicate RT and IGF-1R inhibition had opposing effects on IGF-1/IGF-1R RNA levels in the dentate gyrus. 
1 hour post RT

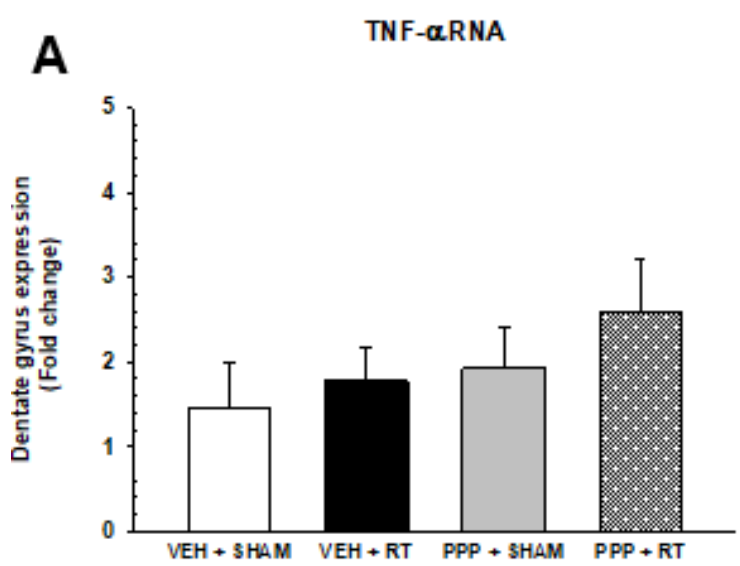

C

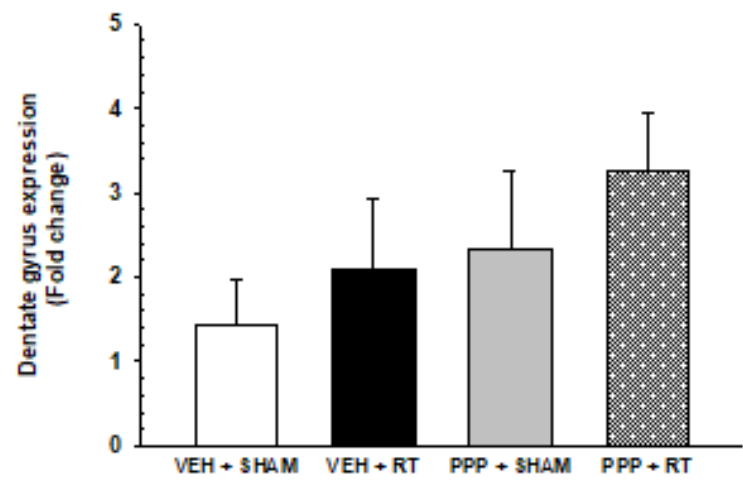

IL -6 RNA

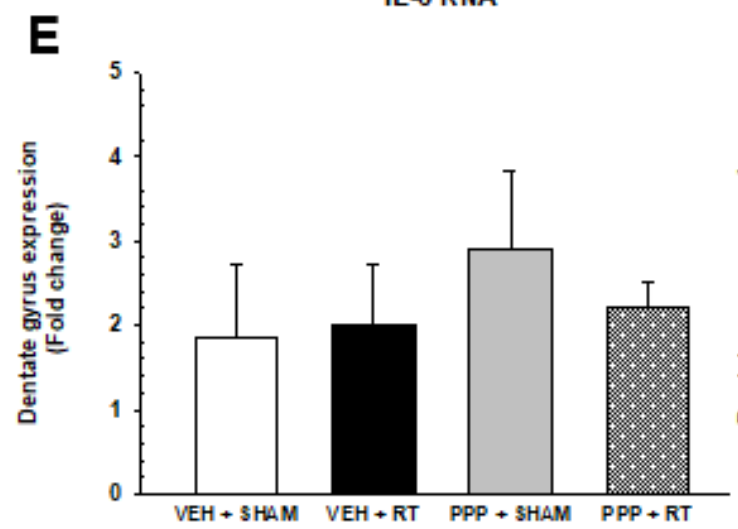

24 hour post RT

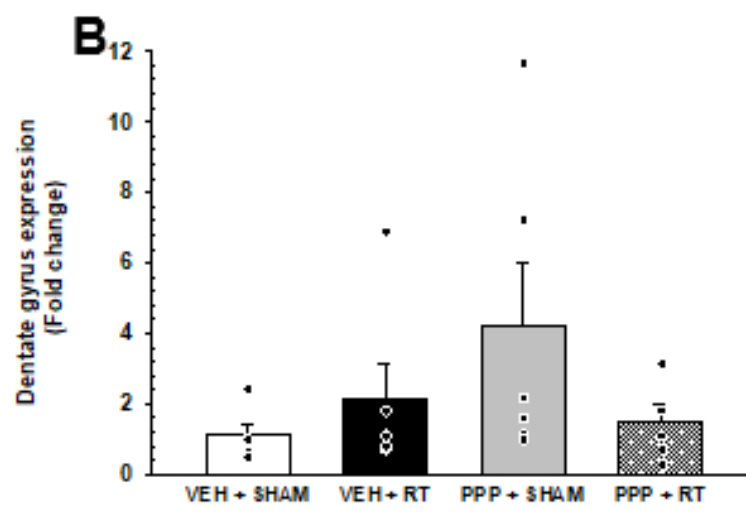

D

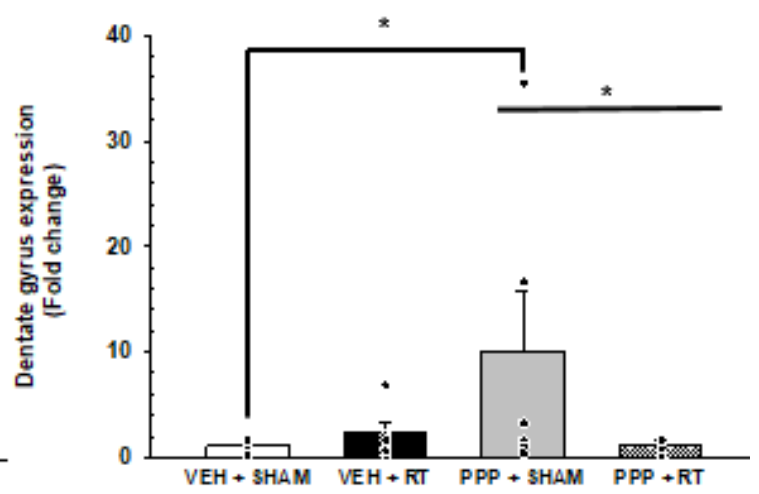

F

IL-6 RNA

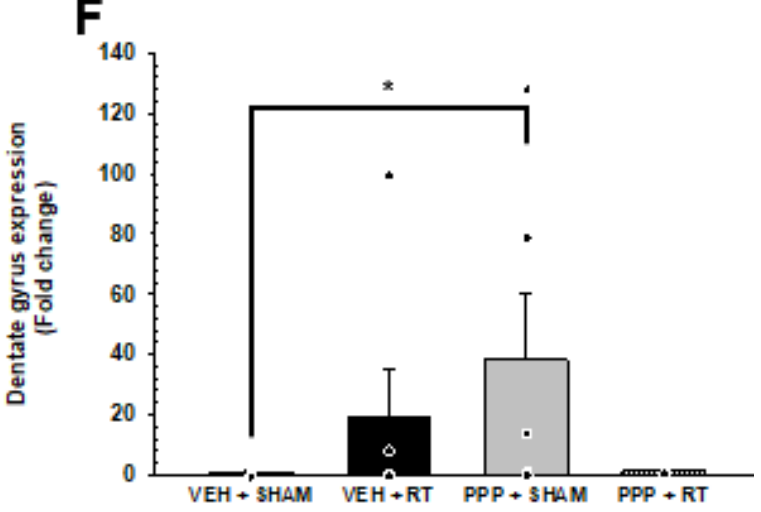

Figure 4.4. PPP injections increase transcriptional markers of neuroinflammation that are ameliorated by RT. A \& B) Fold change of proinflammatory transcript TNF- $\alpha$ in the dentate gyrus one and 24-hours post RT. C \& D) Fold change of proinflammatory transcript IL-1 $\beta$ 
in the dentate gyrus one and 24-hours post RT. E \& F) Fold change of proinflammatory transcript IL-6 in the dentate gyrus one and 24-hours post RT. ${ }^{*}$ denotes a significant effect between groups ( $p \leq .05)$, Two-way ANOVA was used for comparison between groups $(n=5-6)$. Holm Sidak was used post hoc for multiple comparison procedure. RNA is expressed as fold change normalized to VEH + SHAM. 
Resistance-exercise training attenuated neuroinflammation induced by IGF-1R inhibitor in the dentate gyrus

To assess the effects of both RT and PPP injections on neuroinflammation, a two-way ANOVA was used to examine the effect of LPS and RT on inflammatory transcripts in the dentate gyrus at one and 24-hours post RT. Analysis of IL-1 $\beta$ (interleukin-1- beta) 24hours post RT revealed an interaction between RT and PPP injections, $F(1,19)=5.432$, $\mathrm{p}=.031$. Post hoc analysis revealed an increase in IL-1 $\beta$ transcript in PPP + SHAM $(M=$ 2.008, SEM $=0.594)$ compared to VEH + SHAM $(M=-0.001, \operatorname{SEM}=0.594), t=2.394, p$ $=.027$. Post hoc analysis also revealed a decrease in IL-1 $\beta$ transcript in PPP + RT $(M=$ $0.0860, \mathrm{SEM}=0.650)$ compared to PPP + SHAM $(M=2.008, \operatorname{SEM}=0.594), t=2.378, p$ $=0.028$. (Fig. 4.4d). Analysis of IL-6 24-hours post RT revealed a revealed an interaction between RT and PPP injections, $F(1,19)=4.970, p=.038$. Post hoc analysis revealed an increase in IL-6 transcript in PPP + SHAM $(M=3.197$, SEM = 0.913) compared to VEH + SHAM $(M=-0.000$, SEM $=0.913), t=2.475, p=.023$ (Fig. 4.4f). These data indicate that IGF-1R inhibitor increased neuroinflammation in the dentate gyrus that RT attenuated.

\section{Discussion}

Clinical studies propose RT as a suitable intervention to mitigate $\mathrm{MCl}$ (170). Attempts to understand the underlying mechanism have proposed increased IGF-1 signaling and reduced neuroinflammation as potential mechanisms for the therapeutic benefits of RT $(109,204)$. Yet, the in depth causal mechanistic studies are needed. Due to the neuroprotective effect of IGF-1 on neuroinflammation and cognition (205), we hypothesized inhibiting IGF-1 signaling in the dentate gyrus during RT would attenuate the cognitive benefits of RT. Inhibition of IGF-1R signaling during the first RT workout attenuated recognition memory. However, the attenuated recognition memory did not last, as IGF-1R inhibition showed no effect on recognition memory after three weeks of RT. We 
found RT improved spatial memory, regardless of IGF-1R inhibition in the dentate gyrus. Additionally, we found RT and IGF-1R inhibition both improved cognitive flexibility. Combined these data indicate IGF-1R inhibition in the dentate gyrus during RT has differential effects depending training status (acute verse chronic) and the type of cognition (i.e. recognition memory, spatial memory, or cognitive flexibility).

\section{PPP inhibitor attenuated recognition memory improvement after first RT workout}

In the present study, we found PPP injections during RT attenuated recognition memory after one RT workout, while having no effect after three weeks of RT. These data indicate IGF-1 signaling may be important for cognition during the initial stressors of RT, but has little relevancy after multiple RT workouts. This paradoxical interaction between IGF-1R inhibition after acute verse chronic RT could be explained by the training adaptation that takes place after multiple RT workouts. Acute bouts of RT increases inflammatory cytokines in the blood, which are attenuated after multiple RT workouts (206). Therefore, one possible explanation of the results above would be IGF-1 signaling limits the neuroinflammatory response after an acute bout of RT, which is not required after the chronic training adaptation has occurred. IGF-1R phosphorylation limiting the neuroinflammatory response after an acute bout of RT is consistent with the literature as IGF-1 is reported to be ant-inflammatory, acting directly on microglia and astrocytes to inhibit cytokine production or indirectly by improving blood brain barrier efficiency (123, 207, 208). IGF-1 exerting anti-inflammatory effects is also consistent with our findings where IGF-1R inhibition increased neuroinflammatory transcripts in the dentate gyrus, which were attenuated by three weeks of RT. However, it should be noted that IGF-1 has also been reported to exert proinflammatory effects and IGF-1R inhibition to exert antiinflammatory in some studies $(209,210)$, suggesting model and dosage may prove important in teasing out IGF-1 effects on neuroinflammation. 
Three weeks of RT improves recognition memory, regardless of PPP injections

Similar to others (102), we found an improvement in recognition memory after three weeks of RT, despite IGF-1R inhibition. Similarly, we also observed an improvement in spatial memory from RT, independent of IGF-1R inhibition. These findings would indicate that increased IGF-1 signaling is not required for the improvement in recognition or spatial memory after multiple RT workouts. Considering systemic IGF-1 has been shown to improve cognition, we hypothesized IGF-1R inhibition would be detrimental for cognition (205). However, we found IGF-1R inhibition in the dentate gyrus was not able to further impair cognition in rats given LPS. The discrepancies between the two results could be related to the difference in the route in which IGF-1 or IGF-1 inhibition was given. As Quipildor et al. has shown brain-specific overexpression of IGF-1 in 24-month-old mice was not sufficient to improve cognition, yet intranasal treatment of IGF-1 was able to improve cognition (211). Thereby, suggesting the beneficial effects of IGF-1 on cognition are possibly not regulated by the brain itself, but possibly by another tissue, perhaps the blood brain barrier, which could help explain why there was not a detrimental effect on cognition from IGF-1 inhibition.

\section{$R T$ and PPP injections improve cognitive flexibility, independent of one another}

An unpredicted finding in this study was that RT and PPP injection both improved cognitive flexibility, independent of each other. IGF-1 overexpression can improve cognitive flexibility by enhancing adult hippocampal neurogenesis (212). We have previously shown six weeks RT can increase IGF-1 signaling and proliferation, which could account for the improved cognitive flexibility by RT (172). To our knowledge, this is the first study to report IGF-1R inhibition improved cognitive flexibility. Although IGF-1 signaling is classically thought of as neuroprotective, studies have also proposed reduced IGF-1 signaling as 
neuroprotective. For example, neuronal IGF-1R knock out alleviated AD pathology, reduced amyloid plaques and diminished neuroinflammation (125). Additionally, excessive IGF-1 signaling can be detrimental where excessive hippocampal IGF-1R signaling has been suggested to contribute to AD pathology (213). Therefore, IGF-1R inhibition improving cognition is supported by the literature. Alternatively, changes in cognitive flexibility is primarily attributed to the prefrontal cortex (214). Therefore, RT and/or IGF-1R inhibition could be exerting their beneficial effects indirectly by modulating cognitive flexibility through projections from the dentate gyrus to the prefrontal cortex.

$R T$ and PPP injections differentially alter IGF-1 signaling in the dentate gyrus PPP is a selective inhibitor for IGF-1R reported to specifically inhibit the tyrosine residue Y1136 of the internal beta subunit of IGF-1R, without interfering with the insulin receptor (201, 215). Here, we find ICV injections of PPP decrease phosphorylation of IGF-1R (Tyr 1135/1136) in the dentate gyrus of female rats. Our findings are consistent with the literature, as others have found similar decreases in phosphorylation of IGF-1R in the liver after intraperitoneal injections of PPP (210). The present study also revealed PPP injections increased IGF-1/IGF-1R transcripts in the dentate gyrus approximately 1 -hour post injection. Similar observations come from Vasilcanu et al. who observed increased IGF-1R mRNA after exposing malignant cell lines to PPP (216). We also found RT decreased IGF-1/IGF-1R RNA levels in the dentate gyrus 1-hour post RT. Consistent with our findings; studies in skeletal muscle have reported IGF-1 mRNA levels to decrease 1hour post RT (217). These differentially altered IGF-1/IGF-1R RNA levels from RT and PPP injections could be due to the negative regulation of IGF-1 treatment on IGF-1R RNA. IGF-1 has been reported to downregulate IGF-1R transcriptional activity by reducing premRNA and mRNA levels in parallel without altering IGF-1R mRNA stability (218). Due to the opposing actions on IGF-1/IGF-1R RNA from RT and PPP injections, combined with 
the observed inverse relationship between IGF-1 treatment and IGF-1R mRNA, we postulate RT could have increased IGF-1R phosphorylation at a time point other than the two measured. RT-induced IGF-1R phosphorylation is consistent with our previous studies as well as others $(104,109,172)$.

\section{Conclusion}

In the present study, we find inhibition of IGF-1R phosphorylation in the dentate gyrus during the first RT workout attenuated recognition memory, which was not present after three weeks of RT. Three weeks of RT improved spatial memory and cognitive flexibility, regardless of IGF-1R inhibition. Additionally, these studies revealed IGF-1R inhibition could be beneficial for some types of cognition, as IGF-1R inhibition improved cognitive flexibility. Finally, these studies revealed IGF-1R inhibition exacerbated LPS-induced neuroinflammation, which was ameliorated by RT. Taken together; we find cognitive improvements from chronic RT are preserved in the face of IGF-1R inhibition. Considering both RT and IGF-1R inhibition benefited cognition in slightly different ways, RT and IGF1 inhibition could work synergistically to improve cognition. 


\section{Chapter five: Conclusions \& Future Directions}

\section{Dissertation overview}

The aim of this dissertation was to examine underlying mechanisms behind the therapeutic effects of RT on $\mathrm{MCl}$. To study the neuro-molecular changes that occur with resistance-exercise training, I used ladder climbing in Wistar rats to evaluate the dentate gyrus under physiological and pathological conditions. Cognitive behavioral assays connected molecular changes in the dentate gyrus to neurocognitive function. In a series of experiments, I tested a potential mechanism by which RT could ameliorate $\mathrm{MCl}$, where RT increases total IGF-1R protein and IGF-1R signaling, leading to an amelioration of LPS-induced neuroinflammation, subsequently resulting in cognitive improvement. From these experiments, I gained valuable insights summarized in the figure below. (Fig. 5.1).

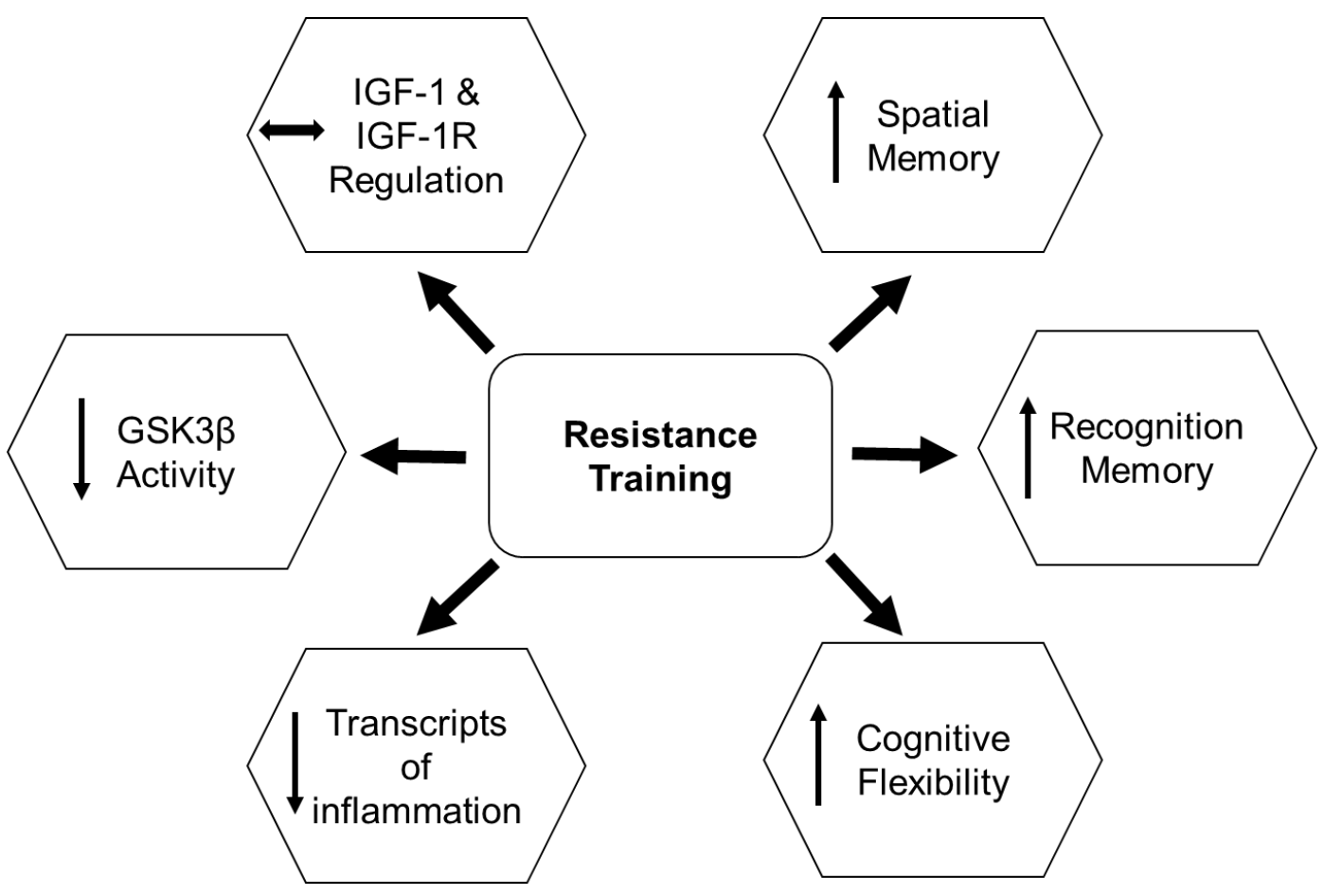

Figure 5.1 Effects of RT that were consistently found during my dissertation thesis. 
In chapter 2, I established a rat model $\mathrm{RT}$ and $\mathrm{MCl}$ in the Booth lab to recapitulate the clinical literature that suggest RT can ameliorate $\mathrm{MCI}(170)$, I uncovered an association between RT and IGF-1 signaling in the dentate gyrus, and revealed RT increases total IGF-1R.

In chapter 3, I found three weeks of RT ameliorates/improves multiple forms of cognition, I showed RT could ameliorate LPS-induced neuroinflammation in a time dependent manner, and I exposed that RT regulates IGF-1/IGF-1R locally in the dentate gyrus.

In chapter 4, I demonstrated that inhibition of IGF-1R signaling during acute RT could attenuate recognition memory, I exposed that inhibition of IGF-1R was not sufficient to attenuate spatial or recognition memory after chronic RT, and I revealed IGF-1R inhibition could improve cognitive flexibility. Additionally, I found IGF-1R inhibition elevates neuroinflammation that was attenuated by RT.

Culmination of these studies uncovered RT-induced changes in the dentate gyrus that revealed insights into the pathophysiology of neuroinflammation and cognitive impairment. Therefore, I believe these findings present my scientific contribution to fields related to exercise physiology, neuroinflammation, and neurocognitive function, by revealing insights into the molecular mechanisms involved in RT-induced amelioration of neuroinflammation and $\mathrm{MCl}$. Additionally, these findings offer IGF-1 inhibition as a potential new area of study to fuel therapies to improve cognition.

Twelve accumulated minutes of RT in the form of ladder climbing improves multiple forms cognition under physiological and pathological conditions in Wistar rats

To recapitulate the clinical literature that indicate $\mathrm{RT}$ can ameliorate $\mathrm{MCI}(170)$, I developed an individualized model of progressive ladder climbing based off earlier reports 
from others in the literature $(143,219-221)$. The ladder climbing model increased maximum lifting capacity, hind limb muscle wet weight, and lean muscle mass (Fig. 2.4), which corresponds to the human RT protocols found in the literature $(145,222,223)$. These data indicate ladder climbing as a translatable rat model of RT.

In chapter 2, I found six weeks of RT was able to ameliorate cognitive deficits induced by LPS, yet changes in phosphoprotein were found as early as one week (3 total workouts) of RT, suggesting RT benefits on cognition could come much earlier than six weeks. Chapters three and four tested the hypothesis that only three weeks of RT (the minimum required to assess multiple forms of cognition) could improve cognitive functions induced by LPS. Three weeks of RT (8 workouts total), where each workout was eight $100-\mathrm{cm}$ weighted climbs up a ladder, spanning less than ten seconds on average, is approximately 640 seconds or twelve minutes that the muscle is contracting during ladder climbing for the entire length of the study, which was approximately $0.04 \%$ of the duration within the three weeks. Chapter three found ICV-LPS injections caused spatial and recognition memory deficits that three weeks of RT could restore. Chapter three also revealed three weeks RT improved in cognitive flexibility, regardless of LPS injections. Additionally, chapter four exposed that the effects of three weeks RT on these three forms of cognition could be replicated. It also uncovered a potential synergistic effect between RT and IGF1 inhibition, as the group that had both seemed to do significantly better on almost every cognitive measure taken. Collectively, these results would propose those twelve minutes of RT was a sufficient stimulus to improve multiple forms of cognition.

\section{$R T$ increases IGF-1R and subsequent downstream signaling in the dentate gyrus}

RT had been shown to increase IGF-1R signaling in hippocampus both others (109). IGF$1 \mathrm{R}$ is a tyrosine kinase receptor composed of two ligand-binding extracellular $\alpha$-subunits, 
two transmembrane $\beta$-subunits, and an internal C-terminal domain (224). Upon ligandreceptor interaction, three tyrosine residues within the kinase domain (Tyr1131, Tyr1135, and Tyr1136) are activated causing a conformational shift allowing for substrate access to the ATP binding site (225), which leads to downstream PI3K/Akt and Raf/MEK/ERK signaling (226). In my initial assessment of RT-induced changes in the dentate gyrus, I found tyrosine $1135 / 1136$ residue phosphorylation of the IGF-1R, which are two of the three earliest phosphorylated residues required for IGF-1R activation (225), increased after six weeks of RT. Additionally, I found six weeks of RT increased AKT phosphorylation at the serine 473 residue and increased phosphorylation of GSK3 $\beta$ at the serine nine residue (Fig. 2.6). Since IGF-1R phosphorylation stimulates AKT leading to GSK3 $\beta$ phosphorylation at the serine nine residue that deactivates $\operatorname{GSK} 3 \beta(226,227)$, which is reported to be ameliorate neuroinflammation and cognitive impairment (191), I postulated in chapter two a potential mechanism by which RT could ameliorate $\mathrm{MCl}$.

Further evaluation of RT under non-pathological conditions revealed enhanced ERK phosphorylation at threonine 202 and tyrosine 204 residues and again showed an increase in GSK3 $\beta$ phosphorylation after just one week (3 workouts) of RT. ERK and GSK3 $\beta$ signaling can be enhanced by multiple signaling molecules (228-230). However, the literature base for RT-induced IGF-1 signaling combined with my own data lead me to believe IGF-1R phosphorylation may be responsible for the increases in ERK and GSK3 $\beta$ phosphorylation $(109,230,231)$. Regardless if IGF-1 or another signaling molecule was responsible for RT-induced activation of ERK and GSK3 $\beta$, these data suggested molecular changes that could promote cognitive benefits occurred earlier than after six weeks of RT. Therefore, my next experiments had to focus on a shortened RT timeframe. In chapter 3, I extended my findings of associated changes in the dentate gyrus following RT, by shortening the RT protocol to three weeks and adding a 1-hour time point (in addition to 24-hour post RT time point). Three weeks of RT did not increase IGF-1R 
phosphorylation. However, I did observe an increase in total IGF-1R at 24 hours post-RT (Fig. 3.5), similar to that of six weeks RT (Fig. 2.6). Chapter three experiments also showed IGF-1 and IGF-1R RNA were decreased 1-hour post RT. IGF-1 has been reported to regulate the IGF-1R, where exogenous IGF-1 has been shown to reduce IGF-1 and IGF-1R mRNAs in all brain cell types in cell culture (187). Therefore, an increase in total IGF-R at 24-hours post RT and the decreased IGF-1/IGF-1R transcripts could have resulted from an RT-induced increase in IGF-1R phosphorylation at an earlier time point not measured. Interpretation of chapters two and three in the context of the literature, suggested RT could be increasing IGF-1R phosphorylation in the dentate gyrus at three weeks RT. Additionally, chapter four further supports this interpretation as IGF-1 inhibition and RT differentially altered IGF-1/IGF-1R transcripts (Fig. 3.6), suggesting IGF-1 inhibition and RT have opposing actions on IGF-1 signaling in the dentate gyrus.

Further support for RT-induced IGF-1 signaling in the dentate gyrus comes from the phosphoprotein changes observed in chapter 3 . Reports of the negative feedback loop that IGF-1/AKT signaling has on its own receptor indicate the decrease in AKT phosphorylation 1-hour post RT could potentially facilitate the increase in total IGF-1R observed 24-hours post RT (Fig. 3.5) (190). Additionally, three weeks of RT rescued deficits in GSK3 $\beta$ phosphorylation induced by LPS. These findings are consistent with chapter 2, where one and six weeks RT increased GSK3 $\beta$ phosphorylation. Considering GSK3 $\beta$ phosphorylation is downstream of IGF-1R phosphorylation, these data could be in support of RT-induced IGF-1 signaling after three weeks RT. However, GSK3 $\beta$ phosphorylation is also downstream of AKT; therefore, it is odd that GSK3 $\beta$ phosphorylation is up at the same time point that AKT signaling is down. One possible explanation could be that GSK3 $\beta$ can be phosphorylated under a separate exerciseinduced mechanism. Aschenbach et al. has demonstrated exercise induces dephosphorylation of $\beta$-catenin associated with increased GSK3 $\beta$ phosphorylation at the 
serine nine residue, independent of insulin signaling in rat skeletal muscle (232). Although exercise and insulin can both deactivate GSK3 $\beta$, it seems GSK3 $\beta$ deactivation from exercise is not dependent on AKT signaling, which could help interpret the increased GSK3 $\beta$ phosphorylation observed in chapter 3.

\section{RT decreases LPS-induced neuroinflammation in the dentate gyrus in a delayed} time dependent manner

One of the major findings in chapter three is the amelioration in LPS-induced neuroinflammatory transcripts 24-hours post RT after LPS-induced neuroinflammation (Fig 3.3). Only one other group has shown that RT can reduce neuroinflammation, however, that group did not look at other time points during RT (102). Therefore, they were unable to conclude whether the drop in neuroinflammation is time-dependent or not. Based on the findings in chapter 3, I found RT to ameliorate neuroinflammation only at the 24-hour time point (i.e. not at 1-hour) (Fig. 3.3). Thereby, suggesting RT decreases neuroinflammation in the dentate gyrus in a delayed time dependent manner.

Both chapters two and three found GSK3 $\beta$ to increase with RT (Fig 2.6 \& 3.5). Collectively, these chapters reveal a consistent effect where RT increases phosphorylation of GSK3 $\beta$ (serine 9) after 1, 3, and six weeks of RT. Increased GSK3 $\beta$ activity is associated with neuroinflammation and cognitive deficits (191, 192), while reducing GSK3 $\beta$ activity targeting phosphorylation of the serine nine residue seems to be a potential therapeutic to treat neuroinflammation (193). Other studies report increased GSK3 $\beta$ activity mediates microglia and astrocyte inflammatory response, whereby using lithium chloride (known to deactivate GSK3 $\beta$ ) inflammatory responses were ameliorated $(233,234)$. Collectively, these studies support the anti-inflammatory effects of deactivation of GSK3 $\beta$, which strengthen the postulation that RT can ameliorate neuroinflammation. 
Additionally, in chapter 4, I found RT also ameliorated PPP-induced neuroinflammatory transcript IL-1 $\beta$. However, there was no observed drop in neuroinflammatory transcripts between groups that did not receive PPP. Considering all the groups in chapter four received LPS, the groups that did not receive PPP should have resulted in a drop in neuroinflammatory transcripts at the 24 -hour time point, similarly to that in chapter 3. A possible interpretation for the lack of attenuated neuroinflammation by RT might be due to the cannulation (used in chapter 4) verses direct inject surgeries (used in chapter 3). It is possible cannulation surgeries cause additional inflammation that cannot be overcome by RT. Support for this posit can be found in chapter 2, where LPS dosage given was higher and neuroinflammatory transcripts were not attenuated by RT (Fig. 2.2). This would provide a potential reason for RT failing to reduce neuroinflammatory transcripts when PPP was not injected. However, when PPP was injected there was an increase in IL-1 $\beta$ that was then reduced by RT (Fig. 4.4). A possible explanation for this observation could be related to the way by which IGF-1 reduces neuroinflammation. IGF-1 is reported to be ant-inflammatory via direct and indirect mechanisms, where IGF-1 exerts a neuroinflammatory response directly by acting on microglia and astrocytes to inhibit cytokine production, while acting indirectly by reducing blood brain barrier permeability $(207,235)$. Considering exercise has been reported to preserve the integrity of the blood brain barrier by protecting tight junctions (236), it could possibly be the amelioration of PPP-induced neuroinflammation from RT comes from a more efficient blood brain barrier, by restoring permeability of blood brain barrier that was increased from PPP.

In chapter 3, I also found that RT ameliorated LPS-induced increases in GFAP protein expression (Fig 3.4). GFAP is a major protein component of astrocyte intermediate filaments that is commonly used as a measure of astrocyte reactivity during pathological conditions. It should be noted that GFAP is not an absolute marker of astrocyte reactivity, still increases in GFAP are often indicative of reactive astrocyte remodeling (185), a 
phenomenon associated with neuroinflammation (102). Therefore, the amelioration of LPS-induced GFAP protein in the dentate gyrus at 24-hours post RT further supports RT as having anti-inflammatory effects in the dentate gyrus that are time dependent.

\section{IGF-1 signaling reduces neuroinflammation in the dentate gyrus}

IGF-1 signaling and neuroinflammation have a complex relationship. Numerous studies report IGF-1 to have anti-inflammatory effects in the brain $(120,202,237)$. IGF-1 is thought to exert its anti-inflammatory effects by directly reducing cytokine production in microglia and astrocytes and indirectly by reducing blood brain barrier permeability $(207,235)$. On the other hand, IGF-1 has also been reported to reduce neuroinflammation under pathological conditions, such as Alzheimer's disease-like mice models (125). PPP, the IGF-1R inhibitor used in this study, also reports contrasting results. PPP was found to abrogate existing glucagon-like peptide-1-induced reductions in inflammation in primary astrocytes under oxygen-glucose deprivation (238). Yet, PPP was also reported to ameliorate neuroinflammation in an Alzheimer's disease transgenic mouse model (210). To further complicate the above interactions between IGF-1 and neuroinflammation, neuroinflammation is reported to cause IGF- 1 resistance (239). TNF- $\alpha$ and IL-1 $\beta$ are reported to inhibit the IGF-I receptor from phosphorylating insulin receptor substrate-1 without affecting tyrosine kinase activity of the IGF-IR itself (240), which could possibly explain the increased IGF-1R phosphorylation that I observed after ICV-LPS injections in chapter three (Fig. 3.5). I speculate that the IGF-1 signaling could have been increased to compensate for IGF-1 resistance at the insulin receptor substrate-1, which could help clarify the reduced GSK3 $\beta$ phosphorylation after ICV-LPS injections observed in chapter three (Fig. 3.5). Where reduced GSK3 $\beta$ phosphorylation could be a potential artifact of insulin resistance after SHAM training. It is apparent from the literature that IGF-1 signaling has a complicated relationship with neuroinflammation. I believe the studies from my 
dissertation offer insight into the relationship between IGF-1 signaling and neuroinflammation.

Evidence from this dissertation advocates for the former and not the latter, where IGF-1 signaling is anti-inflammatory and not proinflammatory. Supportive evidence of the posit that IGF-1 signaling might be responsible for decreased neuroinflammation comes from the associative data in chapter three and four. I have provided evidence that RT both increases IGF-1R phosphorylation (and downstream signaling) and decreases neuroinflammatory transcripts in the dentate gyrus. Combined these data would provide correlational evidence that IGF-1 signaling from RT could exert anti-inflammatory properties during LPS-induced neuroinflammation. Therefore, my data would be in support of IGF-1 inhibiting neuroinflammation (at least in my model).

Further mechanistic evidence comes from chapter 4, where I inhibited IGF-1R phosphorylation in the dentate gyrus by injecting PPP, a selective IGF-1R inhibitor, to test the hypothesis that IGF-1R phosphorylation is required for RT-induced cognitive improvements. By examining neuroinflammatory transcripts, I also tested a subhypothesis that inhibition of IGF-1R phosphorylation would increase neuroinflammation in the dentate gyrus. PPP is reported to selectively inhibit tyrosine residue 1136 of the IGF$1 R$ beta subunit. Theoretically, this decreases the stability of the ligand-induced conformational change that allows substrate interaction with the ATP-binding site that leads to auto phosphorylation of IGF-1R $(201,215)$. Using phospho-IGF-I receptor $\beta$ (Tyr $1135 / 1136)$ antibody, phosphorylation of tyrosine residues $1135 / 1136$ were decreased after PPP injections at the 1-hour time point (Fig. 4.3). These PPP injections also increased neuroinflammatory transcripts IL-1 $\beta$ and IL-6 RNA at the 24-hour time point (Fig. 4.4), suggesting a loss of IGF-1R phosphorylation was sufficient in my experimental paradigm to increase neuroinflammatory transcripts in vivo. Together, these data suggest 
inhibition of IGF-1R increases neuroinflammation, which would infer that increased IGF-1 signaling might be responsible for decreased neuroinflammation observed with RT.

\section{Neuroinflammation is sufficient to cause cognitive impairment, but reduced neuroinflammation may not necessary to restore cognition}

Chapters two \& three show ICV-LPS injection cause increases in neuroinflammatory transcripts such as TNF- $\alpha$ and IL-1 (Fig. 2.2 \& 3.3). Additionally, ICV-LPS injection was shown to cause deficits in spatial and recognition memory (Fig. $2.5 \& 3.2$ ). The results are consistent with the literature that reports ICV-LPS neuroinflammatory model increases TNF- $\alpha$ and IL-1 $\beta$ in the brain resulting in cognitive impairments (78). In support, systemic IL-1 $\beta$ has been reported to replicate LPS-induced deficits in memory (241). Combined, these studies would suggest neuroinflammation is sufficient to cause cognitive impairments.

Chapter three exposed RT as eliciting anti-inflammatory effects in the brain, reducing both neuroinflammatory transcripts and GFAP protein expression, extending other studies that have found reducing neuroinflammation to improve memory (Fig. 3.4) (242). However, chapter two \& four both found RT to improve cognition despite the elevated neuroinflammatory transcripts (Fig. 2.5 \& 3.2), which would indicate RT-induced amelioration of neuroinflammation is not necessarily required for the improvements in cognition in my model. Further support can be found in chapter 3 , where cognitive flexibility is improved regardless of LPS, where SHAM and RT groups that did not receive LPS had no changes in TNF- $\alpha$ or IL-1 1 , yet RT was still able to increase cognitive flexibility (Fig. 3.2). Similarly, chapter four revealed IGF-1R inhibition improved cognitive flexibility without reducing neuroinflammation (Fig. 4.2 \& 4.4), which also advocates for other neuromolecular mechanisms contributing to the cognitive improvements observed. It is also 
possible that RT is ameliorating neuroinflammation through other interleukins. For instance, IL-10 transcript was elevated after short-term RT in 3xTg AD mice (102). However, this is not to say that reductions in neuroinflammation do not elicit any effects on cognition, as many mechanistic studies have definitively shown reduced neuroinflammation can improve cognition in some models (241-243). Instead, the data in chapters 2-4 represents a unique insight from my dissertation that reduced neuroinflammation is not necessary for cognitive improvements in this experimental paradigm. Although this revelation might seem unfavorable, this is exciting news as it strongly suggests RT and IGF-1R inhibition have other mechanisms (other than amelioration of neuroinflammation), by which each of them restores/improves cognition. Although not studied in my dissertation, long-term exposure to LPS can progress into neurodegeneration (244), supporting the possibility that sustained neuroinflammation is an underlying factor in neurodegenerative dementia. Therefore, LPS could trigger a negative cycle leading to multiple deleterious effects, whereby each one could contribute to cognitive decline (91). RT could potentially ameliorate any number of these deleterious effects to restore cognition. Additionally, it is possible RT could exert its neuroprotective effects through a molecular mechanism independent of LPS-induced changes.

One of these possible molecular mechanisms could involve GSK3 $\beta$ signaling as RT consistently increased phosphorylation of GSK3 $\beta$ at the serine nine residue in my RT model. I bring up deactivation of GSK3 $\beta$ due to its relationship with lithium, which has been shown to stabilize cognitive impairment and is currently under phase four clinical trials to treat the most common form of neurodegenerative dementia: $A D(245)$. Lithium works through multiple pathways to decrease GSK3 $\beta$ activity (246-248), one of which (serine nine phosphorylation) is comparable to how RT deactivated GSK3 $\beta$ in my dissertation. Hence, one of the possible mechanisms that RT could elicit neuroprotective effects could be through decreased GSK3 $\beta$ activity. 


\section{Limitations regarding IGF-1 signaling and cognition to be addressed in future studies}

The efficacy of IGF-1 therapeutics remain controversial today with some suggesting reduced IGF-1 signaling, while others indicate increased IGF-1 signaling as a therapeutic for neurodegeneration and cognition $(125,138)$. Although the relationship between IGF-1 signaling and neuroprotection seems complex, there are multiple possible reasons for the inconsistencies in the literature that should be addressed to better understand the interactions between IGF-1 and cognition. These reasons included both the relative dosage of IGF-1 given and cell-type specific interactions.

Dosage of IGF-1 can provide differential outcomes as IGF-1 has been reported to either protect against or increase LPS-induced damage depending on the dosage of IGF-1 (126). Dosage of IGF-1 having differential outcomes can also be supported by the literature when examining the effects of IGF-1 after central and peripheral injections. As peripheral injections of IGF-1 entering the brain would be regulated by the blood brain barrier, it would seem plausible that peripheral injections would have more beneficial outcomes compared to central administration of IGF-1. This posit is consistent with other studies as Quipildor et al. that found brain-specific overexpression of IGF-1 in 24-month-old mice was not sufficient to improve cognition, yet intranasal treatment of IGF-1 was able to improve cognition (211). The varying response between central and peripheral IGF-1 provides some evidence that regulation of dosage by the blood brain barrier could potentially explain the complex relationship of IGF-1 and neuroprotection.

Examining the literature related to IGF-1 signaling and cognition closely, a pattern starts to emerge, where increased IGF-1 signaling in astrocytes and decreased IGF-1 signaling in neurons both seem to improve cognition. For example, in vitro studies reported IGF-1R in astrocytes (not neurons) to be neuroprotective, while in vivo research demonstrated 
IGF-1 overexpression in astrocytes promotes cognition and neuroprotection (121), suggesting astrocytic IGF-1 signaling to support a healthy microenvironment. This phenomenon can be seen in reverse as well. As astrocyte specific knockdown of IGF-1 receptor (IGF-1R) exacerbates neurodegeneration leading to cognitive dysfunction (249). Conversely, neuronal specific IGF-1R knockdown also has been reported to alleviate Alzheimer's disease pathology, thereby improving cognition $(125,250)$. Combined, these studies promote the idea that the therapeutic effects of IGF-1 signaling could be cellspecific.

\section{Future studies and concluding remarks}

Recent evidence suggested an existence of two different types of reactive astrocytes termed "A1" and "A2" respectively (in analogy to the "M1"/“M2" macrophage nomenclature) $(251,252)$. Experts now postulate that this nomenclature was oversimplified and suggest the A1 and A2 transcriptomes represent only two of many potential astrocyte transcriptomes that can occur in physiological and pathological conditions (185). In this study, I found RT ameliorates astrocyte remodeling induced by LPS (Fig. 3.4). One interesting experiment that would provide a great deal of insight on astrocyte function in pathological and physiological conditions would be to better compare transcriptomes of groups that received LPS and RT with the control group (VEH + SHAM) in chapter 3. This would allow us to determine if RT is reverting the astrocyte back into quiescent state or altering it into a new type of astrocyte all together. If they are not different, I can conclude that there is a reversion back to a similar dominant state after an inflammatory insult. If the groups are different, then the genes found that differ between those two groups could be screened in cell culture to determine if RT creates a subtype of astrocyte that is resistant to LPS-induced neuroinflammation, which could be used to develop new therapies to treat neuroinflammation. 
One interesting finding from this study was that RT improve cognitive flexibility, regardless of LPS injection. Therefore, one area to explore is how RT improves cognitive flexibility. There would be no need for LPS in this study as cognitive flexibility was improved under physiological conditions. I approached the improvements in cognitive flexibility from a dentate gyrus angle. However, the prefrontal cortex is also closely associated with this form of cognition (253), so future studies could determine which brain region is primarily responsible for the enhanced cognitive flexibility observed by RT.

Additionally, IGF-1 inhibition alone also was able to improve cognitive flexibility. Although one of the hypotheses of the study was that increased IGF-1 signaling improves cognition, IGF-1 inhibition lead to similar improvements in cognitive flexibility when compared with RT (Fig. 4.2). One possibility that I cannot rule out with these studies is that RT may restore cognitive flexibility by inhibiting IGF-1 signaling in the dentate gyrus. For this hypothesis to have any merit, a time course of IGF-1 signaling in the dentate gyrus after RT would have to reveal decreases in IGF-1 signaling, similar to that of PPP injections. Although this experiment would be difficult to complete, it could offer interesting insights into how RT could improve more executive functions in the brain.

An additional area that should be studied that would help narrow down the effects from RT on cognition would be to examine the interactions between RT, estrogen, IGF-1, and neuroinflammation. Anti-inflammatory effects of IGF-1 have been reported to be mediated by IGF-1R cross talk with estrogen signaling (202). Considering female Wistar rats were used in all the studies in the dissertation, it is possible estrogen signaling mediates the RT-induced amelioration of neuroinflammation. Therefore, a potential study where female Wistar rats are ovariectomized before RT, then assessed for changes in neuroinflammation, may help tease out whether RT effects on neuroinflammation are mediated through estrogen. 
Even though my experiments in this dissertation thesis has left me with more questions than answers, I believe this work has given valuable insights on how RT, neuroinflammation, and IGF-1 signaling influences cognition. Additionally, my work displays ladder climbing as a translatable model to better understand the underlying neuro-molecular mechanisms of RT, which will be useful as scientists continue to unravel the mysteries behind the physiological and molecular responses that occur with exercise. 


\section{References}

1. Gale SA, Acar D, Daffner KR. Dementia. The American journal of medicine. 2018;131(10):1161-9.

2. Prince M, Bryce R, Albanese E, Wimo A, Ribeiro W, Ferri CP. The global prevalence of dementia: a systematic review and metaanalysis. Alzheimer's \& dementia : the journal of the Alzheimer's Association. 2013;9(1):63-75.e2.

3. Brookmeyer R, Evans DA, Hebert L, Langa KM, Heeringa SG, Plassman BL, et al. National estimates of the prevalence of Alzheimer's disease in the United States. Alzheimer's \& dementia : the journal of the Alzheimer's Association. 2011;7(1):61-73.

4. Meynadier MH, Finkman E, Sturge MD, Worlock JM, Tamargo MC. High-order resonant Raman scattering by combinations and overtones of interface phonons in GaAsAlAs short-period superlattices. Physical review B, Condensed matter. 1987;35(5):251720.

5. Mucke L. Neuroscience: Alzheimer's disease. Nature. 2009;461(7266):895-7.

6. Tan CC, Yu JT, Wang HF, Tan MS, Meng XF, Wang C, et al. Efficacy and safety of donepezil, galantamine, rivastigmine, and memantine for the treatment of Alzheimer's disease: a systematic review and meta-analysis. Journal of Alzheimer's disease : JAD. $2014 ; 41(2): 615-31$.

7. Sugimoto H. [Development of anti-Alzheimer's disease drug based on betaamyloid hypothesis]. Yakugaku zasshi : Journal of the Pharmaceutical Society of Japan. 2010;130(4):521-6.

8. 2016 Alzheimer's disease facts and figures. Alzheimer's \& dementia : the journal of the Alzheimer's Association. 2016;12(4):459-509.

9. Gorelick PB, Scuteri A, Black SE, Decarli C, Greenberg SM, ladecola C, et al. Vascular contributions to cognitive impairment and dementia: a statement for healthcare 
professionals from the american heart association/american stroke association. Stroke. 2011;42(9):2672-713.

10. Kalaria RN. The pathology and pathophysiology of vascular dementia. Neuropharmacology. 2018;134(Pt B):226-39.

11. Auchus AP, Brashear HR, Salloway S, Korczyn AD, De Deyn PP, GassmannMayer C. Galantamine treatment of vascular dementia: a randomized trial. Neurology. $2007 ; 69(5): 448-58$

12. Greenan C, Murphy L, Yu LM, Kehoe PG, Coulthard E, Bath P, et al. A randomised controlled trial of calcium channel blockade (CCB) with Amlodipine For the treatment oF subcortical ischaEmic vasCular demenTia (AFFECT): study protocol. Trials. $2016 ; 17(1): 324$.

13. Kario K, Matsuo T, Hoshide S, Umeda Y, Shimada K. Effect of thrombin inhibition in vascular dementia and silent cerebrovascular disease. An MR spectroscopy study. Stroke. 1999;30(5):1033-7.

14. ladecola C. The pathobiology of vascular dementia. Neuron. 2013;80(4):844-66.

15. Gomperts SN. Lewy Body Dementias: Dementia With Lewy Bodies and Parkinson Disease Dementia. Continuum (Minneapolis, Minn). 2016;22(2 Dementia):435-63.

16. Spillantini MG, Schmidt ML, Lee VM, Trojanowski JQ, Jakes R, Goedert M. Alphasynuclein in Lewy bodies. Nature. 1997;388(6645):839-40.

17. McKeith IG, Dickson DW, Lowe J, Emre M, O'Brien JT, Feldman H, et al. Diagnosis and management of dementia with Lewy bodies: third report of the DLB Consortium. Neurology. 2005;65(12):1863-72.

18. Fearnley JM, Lees AJ. Ageing and Parkinson's disease: substantia nigra regional selectivity. Brain : a journal of neurology. 1991;114 ( Pt 5):2283-301. 
19. Meissner W, Prunier C, Guilloteau D, Chalon S, Gross CE, Bezard E. Time-course of nigrostriatal degeneration in a progressive MPTP-lesioned macaque model of Parkinson's disease. Molecular neurobiology. 2003;28(3):209-18.

20. Aarsland D, Andersen K, Larsen JP, Lolk A, Kragh-Sørensen P. Prevalence and characteristics of dementia in Parkinson disease: an 8-year prospective study. Archives of neurology. 2003;60(3):387-92.

21. Aarsland D, Ballard CG, Halliday G. Are Parkinson's disease with dementia and dementia with Lewy bodies the same entity? Journal of geriatric psychiatry and neurology. 2004;17(3):137-45.

22. Lucetti C, Logi C, Del Dotto P, Berti C, Ceravolo R, Baldacci F, et al. Levodopa response in dementia with lewy bodies: a 1-year follow-up study. Parkinsonism \& related disorders. 2010;16(8):522-6.

23. Verschuur CVM, Suwijn SR, Boel JA, Post B, Bloem BR, van Hilten JJ, et al. Randomized Delayed-Start Trial of Levodopa in Parkinson's Disease. The New England journal of medicine. 2019;380(4):315-24 .

24. Gray R, Ives N, Rick C, Patel S, Gray A, Jenkinson C, et al. Long-term effectiveness of dopamine agonists and monoamine oxidase B inhibitors compared with levodopa as initial treatment for Parkinson's disease (PD MED): a large, open-label, pragmatic randomised trial. Lancet (London, England). 2014;384(9949):1196-205.

25. Haddad F, Sawalha M, Khawaja Y, Najjar A, Karaman R. Dopamine and Levodopa Prodrugs for the Treatment of Parkinson's Disease. Molecules (Basel, Switzerland). 2017;23(1).

26. Fahn S, Oakes D, Shoulson I, Kieburtz K, Rudolph A, Lang A, et al. Levodopa and the progression of Parkinson's disease. The New England journal of medicine. 2004;351(24):2498-508. 
27. Dorsey ER, Sherer T, Okun MS, Bloem BR. The Emerging Evidence of the Parkinson Pandemic. Journal of Parkinson's disease. 2018;8(s1):S3-s8.

28. Sanford AM. Lewy Body Dementia. Clinics in geriatric medicine. 2018;34(4):60315.

29. Castrillo JI, Oliver SG. Alzheimer's as a Systems-Level Disease Involving the Interplay of Multiple Cellular Networks. Methods in molecular biology (Clifton, NJ). 2016;1303:3-48.

30. Petersen RC. Mild cognitive impairment as a diagnostic entity. Journal of internal medicine. 2004;256(3):183-94.

31. Brodaty $\mathrm{H}$, Connors $\mathrm{MH}$, Ames $\mathrm{D}$, Woodward M. Progression from mild cognitive impairment to dementia: a 3-year longitudinal study. The Australian and New Zealand journal of psychiatry. 2014;48(12):1137-42.

32. Bohlken J, Jacob L, Kostev K. Progression of mild cognitive impairment to dementia in German specialist practices. Dementia (London, England). 2019;18(1):38090.

33. Chen $\mathrm{PH}$, Cheng SJ, Lin HC, Lee CY, Chou CH. Risk Factors for the Progression of Mild Cognitive Impairment in Different Types of Neurodegenerative Disorders. Behavioural neurology. 2018;2018:6929732.

34. Morris JC. Revised criteria for mild cognitive impairment may compromise the diagnosis of Alzheimer disease dementia. Archives of neurology. 2012;69(6):700-8.

35. Petersen RC, Roberts RO, Knopman DS, Boeve BF, Geda YE, Ivnik RJ, et al. Mild cognitive impairment: ten years later. Archives of neurology. 2009;66(12):1447-55.

36. Petersen RC, Smith GE, Waring SC, Ivnik RJ, Tangalos EG, Kokmen E. Mild cognitive impairment: clinical characterization and outcome. Archives of neurology. $1999 ; 56(3): 303-8$. 
37. Lu D, Popuri K, Ding GW, Balachandar R, Beg MF. Multimodal and Multiscale Deep Neural Networks for the Early Diagnosis of Alzheimer's Disease using structural MR and FDG-PET images. Scientific reports. 2018;8(1):5697.

38. Aerts L, Heffernan M, Kochan NA, Crawford JD, Draper B, Trollor JN, et al. Effects of $\mathrm{MCl}$ subtype and reversion on progression to dementia in a community sample. Neurology. 2017;88(23):2225-32.

39. Maltais M, Rolland Y, Haÿ PE, Armaingaud D, Cestac P, Rouch L, et al. The Effect of Exercise and Social Activity Interventions on Nutritional Status in Older Adults with Dementia Living in Nursing Homes: A Randomised Controlled Trial. The journal of nutrition, health \& aging. 2018;22(7):824-8.

40. Montero-Odasso M, Almeida QJ, Burhan AM, Camicioli R, Doyon J, Fraser S, et al. SYNERGIC TRIAL (SYNchronizing Exercises, Remedies in Gait and Cognition) a multi-Centre randomized controlled double blind trial to improve gait and cognition in mild cognitive impairment. BMC geriatrics. 2018;18(1):93.

41. Suzuki T, Shimada H, Makizako H, Doi T, Yoshida D, Ito K, et al. A randomized controlled trial of multicomponent exercise in older adults with mild cognitive impairment. PloS one. 2013;8(4):e61483.

42. Shimada H, Doi T, Lee S, Makizako H. Reversible predictors of reversion from mild cognitive impairment to normal cognition: a 4-year longitudinal study. Alzheimer's research \& therapy. 2019;11(1):24.

43. de Souto Barreto P, Demougeot L, Vellas B, Rolland Y. Exercise Training for Preventing Dementia, Mild Cognitive Impairment, and Clinically Meaningful Cognitive Decline: A Systematic Review and Meta-analysis. The journals of gerontology Series A, Biological sciences and medical sciences. 2018;73(11):1504-11. 
44. Krell-Roesch J, Feder NT, Roberts RO, Mielke MM, Christianson TJ, Knopman DS, et al. Leisure-Time Physical Activity and the Risk of Incident Dementia: The Mayo Clinic Study of Aging. Journal of Alzheimer's disease : JAD. 2018;63(1):149-55.

45. Grande G, Vanacore N, Maggiore L, Cucumo V, Ghiretti R, Galimberti D, et al. Physical activity reduces the risk of dementia in mild cognitive impairment subjects: a cohort study. Journal of Alzheimer's disease : JAD. 2014;39(4):833-9.

46. Shimada H, Makizako H, Doi T, Park H, Tsutsumimoto K, Verghese J, et al. Effects of Combined Physical and Cognitive Exercises on Cognition and Mobility in Patients With Mild Cognitive Impairment: A Randomized Clinical Trial. Journal of the American Medical Directors Association. 2018;19(7):584-91.

47. Corey-Bloom J. Treatment trials in aging and mild cognitive impairment. Current topics in behavioral neurosciences. 2012;10:347-56.

48. Levey A, Lah J, Goldstein F, Steenland K, Bliwise D. Mild cognitive impairment: an opportunity to identify patients at high risk for progression to Alzheimer's disease. Clinical therapeutics. 2006;28(7):991-1001.

49. Mathys H, Davila-Velderrain J, Peng Z, Gao F, Mohammadi S, Young JZ, et al. Author Correction: Single-cell transcriptomic analysis of Alzheimer's disease. Nature. 2019;571(7763):E1.

50. Seo SW, Ahn J, Yoon U, Im K, Lee JM, Tae Kim S, et al. Cortical thinning in vascular mild cognitive impairment and vascular dementia of subcortical type. Journal of neuroimaging : official journal of the American Society of Neuroimaging. 2010;20(1):3745.

51. Münch G, Lüth HJ, Wong A, Arendt T, Hirsch E, Ravid R, et al. Crosslinking of alpha-synuclein by advanced glycation endproducts--an early pathophysiological step in Lewy body formation? Journal of chemical neuroanatomy. 2000;20(3-4):253-7. 
52. Stubendorff K, Larsson V, Ballard C, Minthon L, Aarsland D, Londos E. Treatment effect of memantine on survival in dementia with Lewy bodies and Parkinson's disease with dementia: a prospective study. BMJ open. 2014;4(7):e005158.

53. Sawada H, Oeda T, Kohsaka M, Umemura A, Tomita S, Park K, et al. Early use of donepezil against psychosis and cognitive decline in Parkinson's disease: a randomised controlled trial for two years. Journal of neurology, neurosurgery, and psychiatry. 2018;89(12):1332-40.

54. Kruglov LS. The early stage of vascular dementia: significance of a complete therapeutic program. International journal of geriatric psychiatry. 2003;18(5):402-6.

55. Dichgans M, Markus HS, Salloway S, Verkkoniemi A, Moline M, Wang Q, et al. Donepezil in patients with subcortical vascular cognitive impairment: a randomised double-blind trial in CADASIL. The Lancet Neurology. 2008;7(4):310-8.

56. Langa KM, Levine DA. The diagnosis and management of mild cognitive impairment: a clinical review. Jama. 2014;312(23):2551-61.

57. Tschanz JT, Welsh-Bohmer KA, Lyketsos CG, Corcoran C, Green RC, Hayden K, et al. Conversion to dementia from mild cognitive disorder: the Cache County Study. Neurology. 2006;67(2):229-34.

58. Lopez OL, Kuller LH, Becker JT, Dulberg C, Sweet RA, Gach HM, et al. Incidence of dementia in mild cognitive impairment in the cardiovascular health study cognition study. Archives of neurology. 2007;64(3):416-20.

59. Cooper C, Li R, Lyketsos C, Livingston G. Treatment for mild cognitive impairment: systematic review. The British journal of psychiatry : the journal of mental science. 2013;203(3):255-64 .

60. Logan JM, Sanders AL, Snyder AZ, Morris JC, Buckner RL. Under-recruitment and nonselective recruitment: dissociable neural mechanisms associated with aging. Neuron. 2002;33(5):827-40. 
61. Clément F, Gauthier S, Belleville S. Executive functions in mild cognitive impairment: emergence and breakdown of neural plasticity. Cortex; a journal devoted to the study of the nervous system and behavior. 2013;49(5):1268-79.

62. King E, O'Brien J, Donaghy P, Williams-Gray CH, Lawson RA, Morris CM, et al. Inflammation in mild cognitive impairment due to Parkinson's disease, Lewy body disease, and Alzheimer's disease. International journal of geriatric psychiatry. 2019;34(8):1244-50.

63. Kirova AM, Bays RB, Lagalwar S. Working memory and executive function decline across normal aging, mild cognitive impairment, and Alzheimer's disease. BioMed research international. 2015;2015:748212.

64. Fernandez A, Meechan DW, Karpinski BA, Paronett EM, Bryan CA, Rutz HL, et al. Mitochondrial Dysfunction Leads to Cortical Under-Connectivity and Cognitive Impairment. Neuron. 2019;102(6):1127-42.e3.

65. Sheppard O, Coleman MP, Durrant CS. Lipopolysaccharide-induced neuroinflammation induces presynaptic disruption through a direct action on brain tissue involving microglia-derived interleukin one beta. Journal of neuroinflammation. 2019;16(1):106.

66. Wang J, Tan L, Wang HF, Tan CC, Meng XF, Wang C, et al. Anti-inflammatory drugs and risk of Alzheimer's disease: an updated systematic review and meta-analysis. Journal of Alzheimer's disease : JAD. 2015;44(2):385-96.

67. Chen H, Zhang SM, Hernán MA, Schwarzschild MA, Willett WC, Colditz GA, et al. Nonsteroidal anti-inflammatory drugs and the risk of Parkinson disease. Archives of neurology. 2003;60(8):1059-64.

68. Miguel-Álvarez M, Santos-Lozano A, Sanchis-Gomar F, Fiuza-Luces C, ParejaGaleano H, Garatachea N, et al. Non-steroidal anti-inflammatory drugs as a treatment for Alzheimer's disease: a systematic review and meta-analysis of treatment effect. Drugs \& aging. 2015;32(2):139-47. 
69. Harman D. Aging: a theory based on free radical and radiation chemistry. Journal of gerontology. 1956;11(3):298-300.

70. Grimm A, Eckert A. Brain aging and neurodegeneration: from a mitochondrial point of view. Journal of neurochemistry. 2017;143(4):418-31.

71. Leuner K, Schütt T, Kurz C, Eckert SH, Schiller C, Occhipinti A, et al. Mitochondrion-derived reactive oxygen species lead to enhanced amyloid beta formation. Antioxidants \& redox signaling. 2012;16(12):1421-33.

72. Golpich M, Amini E, Mohamed Z, Azman Ali R, Mohamed Ibrahim N, Ahmadiani A. Mitochondrial Dysfunction and Biogenesis in Neurodegenerative diseases: Pathogenesis and Treatment. CNS neuroscience \& therapeutics. 2017;23(1):5-22.

73. Zhao YF, Qiong Z, Zhang JF, Lou ZY, Zu HB, Wang ZG, et al. The Synergy of Aging and LPS Exposure in a Mouse Model of Parkinson's Disease. Aging and disease. 2018;9(5):785-97.

74. Hunter R, Ojha U, Bhurtel S, Bing G, Choi DY. Lipopolysaccharide-induced functional and structural injury of the mitochondria in the nigrostriatal pathway. Neuroscience research. 2017;114:62-9.

75. Bradburn S, Murgatroyd C, Ray N. Neuroinflammation in mild cognitive impairment and Alzheimer's disease: A meta-analysis. Ageing research reviews. 2019;50:1-8.

76. Okello A, Edison P, Archer HA, Turkheimer FE, Kennedy J, Bullock R, et al. Microglial activation and amyloid deposition in mild cognitive impairment: a PET study. Neurology. 2009;72(1):56-62.

77. Batista CRA, Gomes GF, Candelario-Jalil E, Fiebich BL, de Oliveira ACP. Lipopolysaccharide-Induced Neuroinflammation as a Bridge to Understand Neurodegeneration. International journal of molecular sciences. 2019;20(9). 
78. Zhao J, Bi W, Xiao S, Lan X, Cheng X, Zhang J, et al. Neuroinflammation induced by lipopolysaccharide causes cognitive impairment in mice. Scientific reports. 2019;9(1):5790.

79. Cunningham C, Wilcockson DC, Campion S, Lunnon K, Perry VH. Central and systemic endotoxin challenges exacerbate the local inflammatory response and increase neuronal death during chronic neurodegeneration. The Journal of neuroscience : the official journal of the Society for Neuroscience. 2005;25(40):9275-84.

80. King E, O'Brien JT, Donaghy P, Morris C, Barnett N, Olsen K, et al. Peripheral inflammation in mild cognitive impairment with possible and probable Lewy body disease and Alzheimer's disease. International psychogeriatrics. 2019;31(4):551-60.

81. Butterfield DA, Poon HF, St Clair D, Keller JN, Pierce WM, Klein JB, et al. Redox proteomics identification of oxidatively modified hippocampal proteins in mild cognitive impairment: insights into the development of Alzheimer's disease. Neurobiology of disease. 2006;22(2):223-32.

82. Jacquemont T, De Vico Fallani F, Bertrand A, Epelbaum S, Routier A, Dubois B, et al. Amyloidosis and neurodegeneration result in distinct structural connectivity patterns in mild cognitive impairment. Neurobiology of aging. 2017;55:177-89.

83. Suh HS, Zhao ML, Derico L, Choi N, Lee SC. Insulin-like growth factor one and two (IGF1, IGF2) expression in human microglia: differential regulation by inflammatory mediators. Journal of neuroinflammation. 2013;10:37.

84. Doi T, Shimada H, Makizako H, Tsutsumimoto K, Hotta R, Nakakubo S, et al. Association of insulin-like growth factor-1 with mild cognitive impairment and slow gait speed. Neurobiology of aging. 2015;36(2):942-7.

85. Clark LR, Racine AM, Koscik RL, Okonkwo OC, Engelman CD, Carlsson CM, et al. Beta-amyloid and cognitive decline in late middle age: Findings from the Wisconsin 
Registry for Alzheimer's Prevention study. Alzheimer's \& dementia : the journal of the Alzheimer's Association. 2016;12(7):805-14.

86. Ormerod BK, Hanft SJ, Asokan A, Haditsch U, Lee SW, Palmer TD. PPARY activation prevents impairments in spatial memory and neurogenesis following transient illness. Brain, behavior, and immunity. 2013;29:28-38.

87. Horgusluoglu-Moloch E, Nho K, Risacher SL, Kim S, Foroud T, Shaw LM, et al. Targeted neurogenesis pathway-based gene analysis identifies ADORA2A associated with hippocampal volume in mild cognitive impairment and Alzheimer's disease. Neurobiology of aging. 2017;60:92-103.

88. Brown GC. The endotoxin hypothesis of neurodegeneration. Journal of neuroinflammation. 2019;16(1):180.

89. Zhang R, Miller RG, Gascon R, Champion S, Katz J, Lancero M, et al. Circulating endotoxin and systemic immune activation in sporadic amyotrophic lateral sclerosis (sALS). Journal of neuroimmunology. 2009;206(1-2):121-4.

90. Zhan X, Stamova B, Jin LW, DeCarli C, Phinney B, Sharp FR. Gram-negative bacterial molecules associate with Alzheimer disease pathology. Neurology. 2016;87(22):2324-32.

91. Zakaria R, Wan Yaacob WM, Othman Z, Long I, Ahmad AH, Al-Rahbi B. Lipopolysaccharide-induced memory impairment in rats: a model of Alzheimer's disease. Physiological research. 2017;66(4):553-65.

92. Klakotskaia D, Agca C, Richardson RA, Stopa EG, Schachtman TR, Agca Y. Memory deficiency, cerebral amyloid angiopathy, and amyloid- $\beta$ plaques in APP+PS1 double transgenic rat model of Alzheimer's disease. PloS one. 2018;13(4):e0195469.

93. Moosavi Sohroforouzani A, Shakerian S, Ghanbarzadeh M, Alaei H. Treadmill exercise improves LPS-induced memory impairments via endocannabinoid receptors and cyclooxygenase enzymes. Behavioural brain research. 2020;380:112440. 
94. Vancampfort D, Stubbs B, Firth J, Smith L, Swinnen N, Koyanagi A. Associations between handgrip strength and mild cognitive impairment in middle-aged and older adults in six low- and middle-income countries. International journal of geriatric psychiatry. 2019;34(4):609-16.

95. Fiatarone Singh MA, Gates N, Saigal N, Wilson GC, Meiklejohn J, Brodaty H, et al. The Study of Mental and Resistance-exercise training (SMART) study-resistanceexercise training and/or cognitive training in mild cognitive impairment: a randomized, double-blind, double-sham controlled trial. Journal of the American Medical Directors Association. 2014;15(12):873-80.

96. Liu-Ambrose T, Nagamatsu LS, Voss MW, Khan KM, Handy TC. Resistanceexercise training and functional plasticity of the aging brain: a 12-month randomized controlled trial. Neurobiology of aging. 2012;33(8):1690-8.

97. Liu-Ambrose T, Nagamatsu LS, Graf P, Beattie BL, Ashe MC, Handy TC. Resistance-exercise training and executive functions: a 12-month randomized controlled trial. Archives of internal medicine. 2010;170(2):170-8.

98. Li Z, Peng X, Xiang W, Han J, Li K. The effect of resistance-exercise training on cognitive function in the older adults: a systematic review of randomized clinical trials. Aging clinical and experimental research. 2018.

99. Nagamatsu LS, Handy TC, Hsu CL, Voss M, Liu-Ambrose T. Resistance-exercise training promotes cognitive and functional brain plasticity in seniors with probable mild cognitive impairment. Archives of internal medicine. 2012;172(8):666-8.

100. Broadhouse KM, Singh MF, Suo C, Gates N, Wen W, Brodaty H, et al. Hippocampal plasticity underpins long-term cognitive gains from resistance exercise in MCI. Neurolmage Clinical. 2020;25:102182.

101. Mavros Y, Gates N, Wilson GC, Jain N, Meiklejohn J, Brodaty H, et al. Mediation of Cognitive Function Improvements by Strength Gains After Resistance-exercise training 
in Older Adults with Mild Cognitive Impairment: Outcomes of the Study of Mental and Resistance-exercise training. Journal of the American Geriatrics Society. 2017;65(3):5509.

102. Liu Y, Chu JMT, Yan T, Zhang Y, Chen Y, Chang RCC, et al. Short-term resistance exercise inhibits neuroinflammation and attenuates neuropathological changes in $3 \times \mathrm{Tg}$ Alzheimer's disease mice. Journal of neuroinflammation. 2020;17(1):4.

103. Spellman T, Rigotti M, Ahmari SE, Fusi S, Gogos JA, Gordon JA. Hippocampalprefrontal input supports spatial encoding in working memory. Nature. 2015;522(7556):309-14.

104. Cassilhas RC, Lee KS, Venancio DP, Oliveira MG, Tufik S, de Mello MT. Resistance exercise improves hippocampus-dependent memory. Brazilian journal of medical and biological research $=$ Revista brasileira de pesquisas medicas e biologicas. 2012;45(12):1215-20.

105. Codina-Martínez H, Fernández-García B, Díez-Planelles C, Fernández Á F, Higarza SG, Fernández-Sanjurjo M, et al. Autophagy is required for performance adaptive response to resistance-exercise training and exercise-induced adult neurogenesis. Scandinavian journal of medicine \& science in sports. 2020;30(2):238-53.

106. Ruiz JR, Gil-Bea F, Bustamante-Ara N, Rodríguez-Romo G, Fiuza-Luces C, SerraRexach JA, et al. Resistance-exercise training does not have an effect on cognition or related serum biomarkers in nonagenarians: a randomized controlled trial. International journal of sports medicine. 2015;36(1):54-60.

107. Bossers WJ, van der Woude LH, Boersma F, Hortobágyi T, Scherder EJ, van Heuvelen MJ. A 9-Week Aerobic and Strength Training Program Improves Cognitive and Motor Function in Patients with Dementia: A Randomized, Controlled Trial. The American journal of geriatric psychiatry : official journal of the American Association for Geriatric Psychiatry. 2015;23(11):1106-16. 
108. Vilela TC, Muller AP, Damiani AP, Macan TP, da Silva S, Canteiro PB, et al. Strength and Aerobic Exercises Improve Spatial Memory in Aging Rats Through Stimulating Distinct Neuroplasticity Mechanisms. Molecular neurobiology. 2017;54(10):7928-37.

109. Cassilhas RC, Lee KS, Fernandes J, Oliveira MG, Tufik S, Meeusen R, et al. Spatial memory is improved by aerobic and resistance exercise through divergent molecular mechanisms. Neuroscience. 2012;202:309-17.

110. Lan Y, Huang Z, Jiang Y, Zhou X, Zhang J, Zhang D, et al. Strength exercise weakens aerobic exercise-induced cognitive improvements in rats. PloS one. 2018;13(10):e0205562.

111. Suijo K, Inoue S, Ohya $\mathrm{Y}$, Odagiri $\mathrm{Y}$, Takamiya $\mathrm{T}$, Ishibashi $\mathrm{H}$, et al. Resistance exercise enhances cognitive function in mouse. International journal of sports medicine. 2013;34(4):368-75.

112. Ding Q, Vaynman S, Akhavan M, Ying Z, Gomez-Pinilla F. Insulin-like growth factor I interfaces with brain-derived neurotrophic factor-mediated synaptic plasticity to modulate aspects of exercise-induced cognitive function. Neuroscience. 2006;140(3):82333.

113. Nokia MS, Lensu S, Ahtiainen JP, Johansson PP, Koch LG, Britton SL, et al. Physical exercise increases adult hippocampal neurogenesis in male rats provided it is aerobic and sustained. The Journal of physiology. 2016;594(7):1855-73.

114. Zigova T, Pencea V, Wiegand SJ, Luskin MB. Intraventricular administration of BDNF increases the number of newly generated neurons in the adult olfactory bulb. Molecular and cellular neurosciences. 1998;11(4):234-45.

115. Huat TJ, Khan AA, Pati S, Mustafa Z, Abdullah JM, Jaafar H. IGF-1 enhances cell proliferation and survival during early differentiation of mesenchymal stem cells to neural progenitor-like cells. BMC neuroscience. 2014;15:91. 
116. Choi SH, Bylykbashi E, Chatila ZK, Lee SW, Pulli B, Clemenson GD, et al. Combined adult neurogenesis and BDNF mimic exercise effects on cognition in an Alzheimer's mouse model. Science (New York, NY). 2018;361(6406).

117. Wang H, Liao S, Geng R, Zheng Y, Liao R, Yan F, et al. IGF-1 signaling via the PI3K/Akt pathway confers neuroprotection in human retinal pigment epithelial cells exposed to sodium nitroprusside insult. Journal of molecular neuroscience : MN. $2015 ; 55(4): 931-40$.

118. Talbot K, Wang HY, Kazi H, Han LY, Bakshi KP, Stucky A, et al. Demonstrated brain insulin resistance in Alzheimer's disease patients is associated with IGF-1 resistance, IRS-1 dysregulation, and cognitive decline. The Journal of clinical investigation. 2012;122(4):1316-38.

119. Torres Aleman I. Insulin-like growth factor-1 and central neurodegenerative diseases. Endocrinology and metabolism clinics of North America. 2012;41(2):395-408, vii.

120. Serhan A, Aerts JL, Boddeke E, Kooijman R. Neuroprotection by Insulin-like Growth Factor-1 in Rats with Ischemic Stroke is Associated with Microglial Changes and a Reduction in Neuroinflammation. Neuroscience. 2020;426:101-14.

121. Chen W, He B, Tong W, Zeng J, Zheng P. Astrocytic Insulin-Like Growth Factor-1 Protects Neurons Against Excitotoxicity. Frontiers in cellular neuroscience. 2019;13:298. 122. Sukhanov S, Higashi Y, Shai SY, Vaughn C, Mohler J, Li Y, et al. IGF-1 reduces inflammatory responses, suppresses oxidative stress, and decreases atherosclerosis progression in ApoE-deficient mice. Arteriosclerosis, thrombosis, and vascular biology. 2007;27(12):2684-90.

123. Bluthé RM, Kelley KW, Dantzer R. Effects of insulin-like growth factor-I on cytokine-induced sickness behavior in mice. Brain, behavior, and immunity. 2006;20(1):57-63. 
124. Hung KS, Tsai SH, Lee TC, Lin JW, Chang CK, Chiu WT. Gene transfer of insulinlike growth factor-I providing neuroprotection after spinal cord injury in rats. Journal of neurosurgery Spine. 2007;6(1):35-46.

125. Gontier G, George C, Chaker Z, Holzenberger M, Aid S. Blocking IGF Signaling in Adult Neurons Alleviates Alzheimer's Disease Pathology through Amyloid-beta Clearance. The Journal of neuroscience : the official journal of the Society for Neuroscience. 2015;35(33):11500-13.

126. Pang Y, Zheng B, Campbell LR, Fan LW, Cai Z, Rhodes PG. IGF-1 can either protect against or increase LPS-induced damage in the developing rat brain. Pediatric research. 2010;67(6):579-84.

127. Li Y, Sun W, Han S, Li J, Ding S, Wang W, et al. IGF-1-Involved Negative Feedback of NR2B NMDA Subunits Protects Cultured Hippocampal Neurons Against NMDA-Induced Excitotoxicity. Molecular neurobiology. 2017;54(1):684-96.

128. Bredesen DE, Amos EC, Canick J, Ackerley M, Raji C, Fiala M, et al. Reversal of cognitive decline in Alzheimer's disease. Aging. 2016;8(6):1250-8.

129. Ransohoff RM. How neuroinflammation contributes to neurodegeneration. Science (New York, NY). 2016;353(6301):777-83.

130. Chiaravalloti ND, DeLuca J. Cognitive impairment in multiple sclerosis. The Lancet Neurology. 2008;7(12):1139-51.

131. Emre M. Dementia associated with Parkinson's disease. The Lancet Neurology. 2003;2(4):229-37.

132. Durães F, Pinto M, Sousa E. Old Drugs as New Treatments for Neurodegenerative Diseases. Pharmaceuticals (Basel, Switzerland). 2018;11(2).

133. Kramer AF, Colcombe S. Fitness Effects on the Cognitive Function of Older Adults: A Meta-Analytic Study-Revisited. Perspectives on psychological science : a journal of the Association for Psychological Science. 2018;13(2):213-7. 
134. Liu L, Zhang Q, Cai $Y$, Sun D, He X, Wang L, et al. Resveratrol counteracts lipopolysaccharide-induced depressive-like behaviors via enhanced hippocampal neurogenesis. Oncotarget. 2016;7(35):56045-59.

135. Fernandes J, Soares JC, do Amaral Baliego LG, Arida RM. A single bout of resistance exercise improves memory consolidation and increases the expression of synaptic proteins in the hippocampus. Hippocampus. 2016;26(8):1096-103.

136. Horwood JM, Dufour F, Laroche S, Davis S. Signalling mechanisms mediated by the phosphoinositide 3-kinase/Akt cascade in synaptic plasticity and memory in the rat. The European journal of neuroscience. 2006;23(12):3375-84.

137. Yamagishi S, Matsumoto T, Yokomaku D, Hatanaka H, Shimoke K, Yamada M, et al. Comparison of inhibitory effects of brain-derived neurotrophic factor and insulin-like growth factor on low potassium-induced apoptosis and activation of p38 MAPK and c-Jun in cultured cerebellar granule neurons. Brain research Molecular brain research. 2003;119(2):184-91.

138. Hu A, Yuan H, Wu L, Chen R, Chen Q, Zhang T, et al. The effect of constitutive over-expression of insulin-like growth factor one on the cognitive function in aged mice. Brain research. 2016;1631:204-13.

139. de Almeida AA, Gomes da Silva S, Lopim GM, Vannucci Campos D, Fernandes J, Cabral FR, et al. Resistance Exercise Reduces Seizure Occurrence, Attenuates Memory Deficits and Restores BDNF Signaling in Rats with Chronic Epilepsy. Neurochemical research. 2017;42(4):1230-9.

140. Grigsby KB, Ruegsegger GN, Childs TE, Booth FW. Overexpression of Protein Kinase Inhibitor Alpha Reverses Rat Low Voluntary Running Behavior. Molecular neurobiology. 2018. 
141. Kim SE, Ko IG, Shin MS, Kim CJ, Jin BK, Hong HP, et al. Treadmill exercise and wheel exercise enhance expressions of neutrophic factors in the hippocampus of lipopolysaccharide-injected rats. Neuroscience letters. 2013;538:54-9.

142. Tyagi E, Agrawal R, Nath C, Shukla R. Influence of LPS-induced neuroinflammation on acetylcholinesterase activity in rat brain. Journal of neuroimmunology. 2008;205(1-2):51-6.

143. Jung S, Ahn N, Kim S, Byun J, Joo Y, Kim S, et al. The effect of ladder-climbing exercise on atrophy/hypertrophy-related myokine expression in middle-aged male Wistar rats. The journal of physiological sciences : JPS. 2015;65(6):515-21.

144. Olver TD, McDonald MW, Klakotskaia D, Richardson RA, Jasperse JL, Melling $\mathrm{CWJ}$, et al. A chronic physical activity treatment in obese rats normalizes the contributions of ET-1 and NO to insulin-mediated posterior cerebral artery vasodilation. Journal of applied physiology (Bethesda, Md : 1985). 2017;122(4):1040-50.

145. Cholewa JM, Rossi FE, MacDonald C, Hewins A, Gallo S, Micenski A, et al. The Effects of Moderate- Versus High-Load Resistance-exercise training on Muscle Growth, Body Composition, and Performance in Collegiate Women. Journal of strength and conditioning research. 2018;32(6):1511-24.

146. Saric J, Lisica D, Orlic I, Grgic J, Krieger JW, Vuk S, et al. Resistance-exercise training Frequencies of three and six Times Per Week Produce Similar Muscular Adaptations in Resistance-Trained Men. Journal of strength and conditioning research. 2018.

147. Cross DA, Alessi DR, Cohen P, Andjelkovich M, Hemmings BA. Inhibition of glycogen synthase kinase-3 by insulin mediated by protein kinase B. Nature. 1995;378(6559):785-9.

148. Gomez O, Sanchez-Rodriguez A, Le M, Sanchez-Caro C, Molina-Holgado F, Molina-Holgado E. Cannabinoid receptor agonists modulate oligodendrocyte 
differentiation by activating PI3K/Akt and the mammalian target of rapamycin (mTOR) pathways. British journal of pharmacology. 2011;163(7):1520-32.

149. Zheng $\mathrm{WH}$, Quirion R. Comparative signaling pathways of insulin-like growth factor-1 and brain-derived neurotrophic factor in hippocampal neurons and the role of the PI3 kinase pathway in cell survival. Journal of neurochemistry. 2004;89(4):844-52.

150. Angers A, Fioravante D, Chin J, Cleary LJ, Bean AJ, Byrne JH. Serotonin stimulates phosphorylation of Aplysia synapsin and alters its subcellular distribution in sensory neurons. The Journal of neuroscience : the official journal of the Society for Neuroscience. 2002;22(13):5412-22.

151. Evergren E, Benfenati F, Shupliakov O. The synapsin cycle: a view from the synaptic endocytic zone. Journal of neuroscience research. 2007;85(12):2648-56.

152. Prelich G, Kostura M, Marshak DR, Mathews MB, Stillman B. The cell-cycle regulated proliferating cell nuclear antigen is required for SV40 DNA replication in vitro. Nature. 1987;326(6112):471-5.

153. Ino H, Chiba T. Expression of proliferating cell nuclear antigen (PCNA) in the adult and developing mouse nervous system. Brain research Molecular brain research. $2000 ; 78(1-2): 163-74$.

154. Kurki P, Ogata K, Tan EM. Monoclonal antibodies to proliferating cell nuclear antigen (PCNA)/cyclin as probes for proliferating cells by immunofluorescence microscopy and flow cytometry. Journal of immunological methods. 1988;109(1):49-59.

155. Kurki P, Vanderlaan M, Dolbeare F, Gray J, Tan EM. Expression of proliferating cell nuclear antigen (PCNA)/cyclin during the cell cycle. Experimental cell research. 1986;166(1):209-19.

156. Thalacker-Mercer A, Stec M, Cui X, Cross J, Windham S, Bamman M. Cluster analysis reveals differential transcript profiles associated with resistance-exercise training- 
induced human skeletal muscle hypertrophy. Physiological genomics. 2013;45(12):499507.

157. Cook MD, Martin SA, Williams C, Whitlock K, Wallig MA, Pence BD, et al. Forced treadmill exercise training exacerbates inflammation and causes mortality while voluntary wheel training is protective in a mouse model of colitis. Brain, behavior, and immunity. 2013;33:46-56.

158. Ke Z, Yip SP, Li L, Zheng XX, Tong KY. The effects of voluntary, involuntary, and forced exercises on brain-derived neurotrophic factor and motor function recovery: a rat brain ischemia model. PloS one. 2011;6(2):e16643.

159. Moraska A, Deak T, Spencer RL, Roth D, Fleshner M. Treadmill running produces both positive and negative physiological adaptations in Sprague-Dawley rats. American journal of physiology Regulatory, integrative and comparative physiology. $2000 ; 279(4): R 1321-9$.

160. Svensson M, Rosvall P, Boza-Serrano A, Andersson E, Lexell J, Deierborg T. Forced treadmill exercise can induce stress and increase neuronal damage in a mouse model of global cerebral ischemia. Neurobiology of stress. 2016;5:8-18.

161. Haffejee IE. Persistent diarrhoea following gastroenteritis. Journal of diarrhoeal diseases research. 1990;8(4):143-6.

162. Hennessy E, Gormley S, Lopez-Rodriguez AB, Murray C, Murray C, Cunningham C. Systemic TNF- $\alpha$ produces acute cognitive dysfunction and exaggerated sickness behavior when superimposed upon progressive neurodegeneration. Brain, behavior, and immunity. 2017;59:233-44.

163. Miao H, Dong Y, Zhang Y, Zheng H, Shen Y, Crosby G, et al. Anesthetic Isoflurane or Desflurane Plus Surgery Differently Affects Cognitive Function in Alzheimer's Disease Transgenic Mice. Molecular neurobiology. 2018;55(7):5623-38. 
164. Wu YT, Beiser AS, Breteler MMB, Fratiglioni L, Helmer C, Hendrie HC, et al. The changing prevalence and incidence of dementia over time - current evidence. Nature reviews Neurology. 2017;13(6):327-39.

165. Stephan BCM, Birdi R, Tang EYH, Cosco TD, Donini LM, Licher S, et al. Secular Trends in Dementia Prevalence and Incidence Worldwide: A Systematic Review. Journal of Alzheimer's disease : JAD. 2018;66(2):653-80.

166. Wessels AM, Tariot PN, Zimmer JA, Selzler KJ, Bragg SM, Andersen SW, et al. Efficacy and Safety of Lanabecestat for Treatment of Early and Mild Alzheimer Disease: The AMARANTH and DAYBREAK-ALZ Randomized Clinical Trials. JAMA neurology. 2020;77(2):199-209.

167. Mintun MA, Lo AC, Duggan Evans C, Wessels AM, Ardayfio PA, Andersen SW, et al. Donanemab in Early Alzheimer's Disease. The New England journal of medicine. 2021. 168. Sanford AM. Mild Cognitive Impairment. Clinics in geriatric medicine. 2017;33(3):325-37.

169. Snyder PJ, Jackson CE, Petersen RC, Khachaturian AS, Kaye J, Albert MS, et al. Assessment of cognition in mild cognitive impairment: a comparative study. Alzheimer's \& dementia : the journal of the Alzheimer's Association. 2011;7(3):338-55.

170. Zhang L, Li B, Yang J, Wang F, Tang Q, Wang S. Meta-analysis: Resistanceexercise training Improves Cognition in Mild Cognitive Impairment. International journal of sports medicine. 2020;41(12):815-23.

171. Frost RA, Nystrom GJ, Lang $\mathrm{CH}$. Tumor necrosis factor-alpha decreases insulinlike growth factor-I messenger ribonucleic acid expression in C2C12 myoblasts via a Jun N-terminal kinase pathway. Endocrinology. 2003;144(5):1770-9.

172. Kelty TJ, Schachtman TR, Mao X, Grigsby KB, Childs TE, Olver TD, et al. Resistance-exercise training ameliorates LPS-induced cognitive impairment concurrent 
with molecular signaling changes in the rat dentate gyrus. Journal of applied physiology (Bethesda, Md : 1985). 2019.

173. Molina-Sotomayor E, Castillo-Quezada H, Martínez-Salazar C, González-Orb M, Espinoza-Salinas A, Gonzalez-Jurado JA. Effects of Progressive Resistance-exercise training on Cognition and IGF-1 Levels in Elder Women Who Live in Areas with High Air Pollution. International journal of environmental research and public health. 2020;17(17). 174. Walf AA, Frye CA. The use of the elevated plus maze as an assay of anxietyrelated behavior in rodents. Nature protocols. 2007;2(2):322-8.

175. Gawel K, Gibula E, Marszalek-Grabska M, Filarowska J, Kotlinska JH. Assessment of spatial learning and memory in the Barnes maze task in rodentsmethodological consideration. Naunyn-Schmiedeberg's archives of pharmacology. 2019;392(1):1-18.

176. Leger M, Quiedeville A, Bouet V, Haelewyn B, Boulouard M, Schumann-Bard P, et al. Object recognition test in mice. Nature protocols. 2013;8(12):2531-7.

177. Paxinos G, Watson C. The Rat Brain in Stereotaxic Coordinates. Academic Press, San Diego. 1997;3rd.

178. Vytal KE, Cornwell BR, Letkiewicz AM, Arkin NE, Grillon C. The complex interaction between anxiety and cognition: insight from spatial and verbal working memory. Frontiers in human neuroscience. 2013;7:93.

179. Dees RL, Kesner RP. The role of the dorsal dentate gyrus in object and objectcontext recognition. Neurobiology of learning and memory. 2013;106:112-7.

180. Li M, Li C, Yu H, Cai X, Shen X, Sun X, et al. Lentivirus-mediated interleukin-1 $\beta$ (IL-1 $\beta$ ) knock-down in the hippocampus alleviates lipopolysaccharide (LPS)-induced memory deficits and anxiety- and depression-like behaviors in mice. Journal of neuroinflammation. 2017;14(1):190. 
181. Izquierdo A, Brigman JL, Radke AK, Rudebeck PH, Holmes A. The neural basis of reversal learning: An updated perspective. Neuroscience. 2017;345:12-26.

182. Garthe A, Behr J, Kempermann G. Adult-generated hippocampal neurons allow the flexible use of spatially precise learning strategies. PloS one. 2009;4(5):e5464.

183. Segabinazi E, Gasperini NF, Faustino AM, Centeno R, Santos ASD, Almeida W, et al. Comparative overview of the effects of aerobic and resistance exercise on anxietylike behavior, cognitive flexibility, and hippocampal synaptic plasticity parameters in healthy rats. Brazilian journal of medical and biological research $=$ Revista brasileira de pesquisas medicas e biologicas. 2020;53(11):e9816.

184. Wilke J, Giesche F, Klier K, Vogt L, Herrmann E, Banzer W. Acute Effects of Resistance Exercise on Cognitive Function in Healthy Adults: A Systematic Review with Multilevel Meta-Analysis. Sports medicine (Auckland, NZ). 2019;49(6):905-16.

185. Escartin C, Galea E, Lakatos A, O'Callaghan JP, Petzold GC, Serrano-Pozo A, et al. Reactive astrocyte nomenclature, definitions, and future directions. Nature neuroscience. 2021;24(3):312-25.

186. Shen J, Wang D, Wang X, Gupta S, Ayloo B, Wu S, et al. Neurovascular Coupling in the Dentate Gyrus Regulates Adult Hippocampal Neurogenesis. Neuron. 2019;103(5):878-90.e3.

187. Trueba-Saiz A, Fernandez AM, Nishijima T, Mecha M, Santi A, Munive V, et al. Circulating Insulin-Like Growth Factor I Regulates Its Receptor in the Brain of Male Mice. Endocrinology. 2017;158(2):349-55.

188. Steen E, Terry BM, Rivera EJ, Cannon JL, Neely TR, Tavares R, et al. Impaired insulin and insulin-like growth factor expression and signaling mechanisms in Alzheimer's disease--is this type three diabetes? Journal of Alzheimer's disease : JAD. 2005;7(1):6380. 
189. Ramlo-Halsted BA, Edelman SV. The natural history of type two diabetes. Implications for clinical practice. Primary care. 1999;26(4):771-89.

190. Qin L, Wang Y, Tao L, Wang Z. AKT down-regulates insulin-like growth factor-1 receptor as a negative feedback. Journal of biochemistry. 2011;150(2):151-6.

191. Ebeid MA, Habib MZ, Mohamed AM, Faramawy YE, Saad SST, EI-Kharashi OA, et al. Cognitive effects of the GSK-3 inhibitor "lithium" in LPS/chronic mild stress rat model of depression: Hippocampal and cortical neuroinflammation and tauopathy. Neurotoxicology. 2021;83:77-88.

192. Li J, Shi C, Ding Z, Jin W. Glycogen Synthase Kinase $3 \beta$ Promotes Postoperative Cognitive Dysfunction by Inducing the M1 Polarization and Migration of Microglia. Mediators of inflammation. 2020;2020:7860829.

193. Rana AK, Singh D. Targeting glycogen synthase kinase-3 for oxidative stress and neuroinflammation: Opportunities, challenges and future directions for cerebral stroke management. Neuropharmacology. 2018;139:124-36.

194. Boyle PA, Wilson RS, Aggarwal NT, Tang Y, Bennett DA. Mild cognitive impairment: risk of Alzheimer disease and rate of cognitive decline. Neurology. $2006 ; 67(3): 441-5$.

195. Nuzum H, Stickel A, Corona M, Zeller M, Melrose RJ, Wilkins SS. Potential Benefits of Physical Activity in $\mathrm{MCl}$ and Dementia. Behavioural neurology. $2020 ; 2020: 7807856$.

196. de Oliveira Silva F, Ferreira JV, Plácido J, Sant'Anna P, Araújo J, Marinho V, et al. Three months of multimodal training contributes to mobility and executive function in elderly individuals with mild cognitive impairment, but not in those with Alzheimer's disease: A randomized controlled trial. Maturitas. 2019;126:28-33.

197. Nagamatsu LS, Chan A, Davis JC, Beattie BL, Graf P, Voss MW, et al. Physical activity improves verbal and spatial memory in older adults with probable mild cognitive 
impairment: a 6-month randomized controlled trial. Journal of aging research. 2013;2013:861893.

198. Rojas Vega S, Knicker A, Hollmann W, Bloch W, Strüder HK. Effect of resistance exercise on serum levels of growth factors in humans. Hormone and metabolic research $=$ Hormon- und Stoffwechselforschung = Hormones et metabolisme. $2010 ; 42(13): 982-6$.

199. Bjersing JL, Larsson A, Palstam A, Ernberg M, Bileviciute-Ljungar I, Löfgren M, et al. Benefits of resistance exercise in lean women with fibromyalgia: involvement of IGF-1 and leptin. BMC musculoskeletal disorders. 2017;18(1):106.

200. Cassilhas RC, Viana VA, Grassmann V, Santos RT, Santos RF, Tufik S, et al. The impact of resistance exercise on the cognitive function of the elderly. Medicine and science in sports and exercise. 2007;39(8):1401-7.

201. Girnita A, Girnita L, del Prete F, Bartolazzi A, Larsson O, Axelson M. Cyclolignans as inhibitors of the insulin-like growth factor- 1 receptor and malignant cell growth. Cancer research. 2004;64(1):236-42.

202. Yuan LJ, Zhang M, Chen S, Chen WF. Anti-inflammatory effect of IGF-1 is mediated by IGF-1R cross talk with GPER in MPTP/MPP(+)-induced astrocyte activation. Molecular and cellular endocrinology. 2021;519:111053.

203. Antunes M, Biala $G$. The novel object recognition memory: neurobiology, test procedure, and its modifications. Cognitive processing. 2012;13(2):93-110.

204. Hiscox C. Stress and its management. Nursing standard (Royal College of Nursing (Great Britain) : 1987). 1991;5(21):36-40.

205. Wang F, Wang L, Wang Y, Li D, Hu T, Sun M, et al. Exogenous IGF-1 improves cognitive function in rats with high-fat diet consumption. Journal of molecular endocrinology. 2020;64(2):115-23.

206. Calle MC, Fernandez ML. Effects of resistance-exercise training on the inflammatory response. Nutrition research and practice. 2010;4(4):259-69. 
207. Nowrangi DS, McBride D, Manaenko A, Dixon B, Tang J, Zhang JH. rhlGF-1 reduces the permeability of the blood-brain barrier following intracerebral hemorrhage in mice. Experimental neurology. 2019;312:72-81.

208. Labandeira-Garcia JL, Costa-Besada MA, Labandeira CM, Villar-Cheda B, Rodríguez-Perez Al. Insulin-Like Growth Factor-1 and Neuroinflammation. Frontiers in aging neuroscience. 2017;9:365.

209. Renier G, Clément I, Desfaits AC, Lambert A. Direct stimulatory effect of insulinlike growth factor-I on monocyte and macrophage tumor necrosis factor-alpha production. Endocrinology. 1996;137(11):4611-8.

210. Sohrabi M, Floden AM, Manocha GD, Klug MG, Combs CK. IGF-1R Inhibitor Ameliorates Neuroinflammation in an Alzheimer's Disease Transgenic Mouse Model. Frontiers in cellular neuroscience. 2020;14:200.

211. Farias Quipildor GE, Mao K, Hu Z, Novaj A, Cui MH, Gulinello M, et al. Central IGF-1 protects against features of cognitive and sensorimotor decline with aging in male mice. GeroScience. 2019;41(2):185-208.

212. Littlejohn EL, Scott D, Saatman KE. Insulin-like growth factor-1 overexpression increases long-term survival of posttrauma-born hippocampal neurons while inhibiting ectopic migration following traumatic brain injury. Acta neuropathologica communications. 2020;8(1):46.

213. Gazit N, Vertkin I, Shapira I, Helm M, Slomowitz E, Sheiba M, et al. IGF-1 Receptor Differentially Regulates Spontaneous and Evoked Transmission via Mitochondria at Hippocampal Synapses. Neuron. 2016;89(3):583-97.

214. McAlonan K, Brown VJ. Orbital prefrontal cortex mediates reversal learning and not attentional set shifting in the rat. Behavioural brain research. 2003;146(1-2):97-103.

215. Vasilcanu D, Girnita A, Girnita L, Vasilcanu R, Axelson M, Larsson O. The cyclolignan PPP induces activation loop-specific inhibition of tyrosine phosphorylation of 
the insulin-like growth factor-1 receptor. Link to the phosphatidyl inositol-3 kinase/Akt apoptotic pathway. Oncogene. 2004;23(47):7854-62.

216. Vasilcanu D, Weng WH, Girnita A, Lui WO, Vasilcanu R, Axelson M, et al. The insulin-like growth factor-1 receptor inhibitor PPP produces only very limited resistance in tumor cells exposed to long-term selection. Oncogene. 2006;25(22):3186-95.

217. Psilander N, Damsgaard R, Pilegaard H. Resistance exercise alters MRF and IGFI mRNA content in human skeletal muscle. Journal of applied physiology (Bethesda, Md : 1985). 2003;95(3):1038-44.

218. Hernández-Sánchez C, Werner H, Roberts CT, Jr., Woo EJ, Hum DW, Rosenthal SM, et al. Differential regulation of insulin-like growth factor-I (IGF-I) receptor gene expression by IGF-I and basic fibroblastic growth factor. The Journal of biological chemistry. 1997;272(8):4663-70.

219. Hornberger TA, Jr., Farrar RP. Physiological hypertrophy of the FHL muscle following eight weeks of progressive resistance exercise in the rat. Canadian journal of applied physiology = Revue canadienne de physiologie appliquee. 2004;29(1):16-31.

220. Tang L, Gao X, Yang X, Liu C, Wang X, Han Y, et al. Ladder-Climbing Training Prevents Bone Loss and Microarchitecture Deterioration in Diet-Induced Obese Rats. Calcified tissue international. 2016;98(1):85-93.

221. Molanouri Shamsi M, Mahdavi M, Quinn LS, Gharakhanlou R, Isanegad A. Effect of resistance exercise training on expression of Hsp70 and inflammatory cytokines in skeletal muscle and adipose tissue of STZ-induced diabetic rats. Cell stress \& chaperones. 2016;21(5):783-91.

222. Chilibeck PD, Calder AW, Sale DG, Webber CE. A comparison of strength and muscle mass increases during resistance-exercise training in young women. European journal of applied physiology and occupational physiology. 1998;77(1-2):170-5. 
223. Mobley CB, Haun CT, Roberson PA, Mumford PW, Kephart WC, Romero MA, et al. Biomarkers associated with low, moderate, and high vastus lateralis muscle hypertrophy following 12 weeks of resistance-exercise training. PloS one. 2018;13(4):e0195203.

224. LeRoith D, Werner H, Beitner-Johnson D, Roberts CT, Jr. Molecular and cellular aspects of the insulin-like growth factor I receptor. Endocrine reviews. 1995;16(2):143-63. 225. Favelyukis S, Till JH, Hubbard SR, Miller WT. Structure and autoregulation of the insulin-like growth factor one receptor kinase. Nature structural biology. 2001;8(12):105863.

226. Shelton JG, Steelman LS, White ER, McCubrey JA. Synergy between PI3K/Akt and Raf/MEK/ERK pathways in IGF-1R mediated cell cycle progression and prevention of apoptosis in hematopoietic cells. Cell cycle (Georgetown, Tex). 2004;3(3):372-9.

227. Case N, Thomas J, Sen B, Styner M, Xie Z, Galior K, et al. Mechanical regulation of glycogen synthase kinase $3 \beta$ (GSK3 $\beta$ ) in mesenchymal stem cells is dependent on Akt protein serine 473 phosphorylation via mTORC2 protein. The Journal of biological chemistry. 2011;286(45):39450-6.

228. Mai L, Jope RS, Li X. BDNF-mediated signal transduction is modulated by GSK3beta and mood stabilizing agents. Journal of neurochemistry. 2002;82(1):75-83. 229. Liu J, Yang J, Xu Y, Guo G, Cai L, Wu H, et al. Roscovitine, a CDK5 Inhibitor, Alleviates Sevoflurane-Induced Cognitive Dysfunction via Regulation Tau/GSK3 $\beta$ and ERK/PPARY/CREB Signaling. Cellular physiology and biochemistry : international journal of experimental cellular physiology, biochemistry, and pharmacology. 2017;44(2):423-35. 230. Subramaniam S, Shahani N, Strelau J, Laliberté C, Brandt R, Kaplan D, et al. Insulin-like growth factor one inhibits extracellular signal-regulated kinase to promote neuronal survival via the phosphatidylinositol 3-kinase/protein kinase A/c-Raf pathway. 
The Journal of neuroscience : the official journal of the Society for Neuroscience. $2005 ; 25(11): 2838-52$.

231. Taylor LW, Wilborn CD, Kreider RB, Willoughby DS. Effects of resistance exercise intensity on extracellular signal-regulated kinase 1/2 mitogen-activated protein kinase activation in men. Journal of strength and conditioning research. 2012;26(3):599-607.

232. Aschenbach WG, Ho RC, Sakamoto K, Fujii N, Li Y, Kim YB, et al. Regulation of dishevelled and beta-catenin in rat skeletal muscle: an alternative exercise-induced GSK3beta signaling pathway. American journal of physiology Endocrinology and metabolism. 2006;291(1):E152-8.

233. Cao Q, Karthikeyan A, Dheen ST, Kaur C, Ling EA. Production of proinflammatory mediators in activated microglia is synergistically regulated by Notch-1, glycogen synthase kinase (GSK-3ß) and NF-kB/p65 signalling. PloS one. 2017;12(10):e0186764.

234. Li N, Zhang X, Dong H, Zhang S, Sun J, Qian Y. Lithium Ameliorates LPS-Induced Astrocytes Activation Partly via Inhibition of Toll-Like Receptor four Expression. Cellular physiology and biochemistry : international journal of experimental cellular physiology, biochemistry, and pharmacology. 2016;38(2):714-25.

235. Bake S, Selvamani A, Cherry J, Sohrabji F. Blood brain barrier and neuroinflammation are critical targets of IGF-1-mediated neuroprotection in stroke for middle-aged female rats. PloS one. 2014;9(3):e91427.

236. Souza PS, Gonçalves ED, Pedroso GS, Farias HR, Junqueira SC, Marcon R, et al. Physical Exercise Attenuates Experimental Autoimmune Encephalomyelitis by Inhibiting Peripheral Immune Response and Blood-Brain Barrier Disruption. Molecular neurobiology. 2017;54(6):4723-37.

237. Park SE, Dantzer R, Kelley KW, McCusker RH. Central administration of insulinlike growth factor-I decreases depressive-like behavior and brain cytokine expression in mice. Journal of neuroinflammation. 2011;8:12. 
238. Huang J, Liu Y, Cheng L, Li J, Zhang T, Zhao G, et al. Glucagon-like peptide-1 cleavage product GLP-1(9-36) reduces neuroinflammation from stroke via the activation of insulin-like growth factor one receptor in astrocytes. European journal of pharmacology. 2020;887:173581.

239. O'Connor JC, McCusker RH, Strle K, Johnson RW, Dantzer R, Kelley KW. Regulation of IGF-I function by proinflammatory cytokines: at the interface of immunology and endocrinology. Cellular immunology. 2008;252(1-2):91-110.

240. Shen WH, Zhou JH, Broussard SR, Freund GG, Dantzer R, Kelley KW. Proinflammatory cytokines block growth of breast cancer cells by impairing signals from a growth factor receptor. Cancer research. 2002;62(16):4746-56.

241. Chun MW, Olmstead KK, Choi Y, Lee CO, Lee CK, Kim JH, et al. Synthesis and biological activity of 5-hydroxy-4-quinolones and 5-methoxy-4-quinolones as truncated acridones. Archives of pharmacal research. 1998;21(4):445-51.

242. Lan KM, Tien LT, Pang Y, Bhatt AJ, Fan LW. IL-1 receptor antagonist attenuates neonatal lipopolysaccharide-induced long-lasting learning impairment and hippocampal injury in adult rats. Toxicology letters. 2015;234(1):30-9.

243. Kitazawa M, Cheng D, Tsukamoto MR, Koike MA, Wes PD, Vasilevko V, et al. Blocking IL-1 signaling rescues cognition, attenuates tau pathology, and restores neuronal $\beta$-catenin pathway function in an Alzheimer's disease model. Journal of immunology (Baltimore, Md : 1950). 2011;187(12):6539-49.

244. Qin L, Wu X, Block ML, Liu Y, Breese GR, Hong JS, et al. Systemic LPS causes chronic neuroinflammation and progressive neurodegeneration. Glia. 2007;55(5):453-62. 245. Nunes MA, Viel TA, Buck HS. Microdose lithium treatment stabilized cognitive impairment in patients with Alzheimer's disease. Current Alzheimer research. 2013;10(1):104-7. 
246. De Sarno P, Li X, Jope RS. Regulation of Akt and glycogen synthase kinase-3 beta phosphorylation by sodium valproate and lithium. Neuropharmacology. 2002;43(7):1158-64.

247. Klein PS, Melton DA. A molecular mechanism for the effect of lithium on development. Proceedings of the National Academy of Sciences of the United States of America. 1996;93(16):8455-9.

248. Young W. Review of lithium effects on brain and blood. Cell transplantation. 2009;18(9):951-75.

249. Logan S, Pharaoh GA, Marlin MC, Masser DR, Matsuzaki S, Wronowski B, et al. Insulin-like growth factor receptor signaling regulates working memory, mitochondrial metabolism, and amyloid-beta uptake in astrocytes. Molecular metabolism. 2018;9:14155.

250. George C, Gontier G, Lacube P, Francois JC, Holzenberger M, Aid S. The Alzheimer's disease transcriptome mimics the neuroprotective signature of IGF-1 receptor-deficient neurons. Brain : a journal of neurology. 2017;140(7):2012-27.

251. Liddelow SA, Guttenplan KA, Clarke LE, Bennett FC, Bohlen CJ, Schirmer L, et al. Neurotoxic reactive astrocytes are induced by activated microglia. Nature. 2017;541(7638):481-7.

252. Zamanian JL, Xu L, Foo LC, Nouri N, Zhou L, Giffard RG, et al. Genomic analysis of reactive astrogliosis. The Journal of neuroscience : the official journal of the Society for Neuroscience. 2012;32(18):6391-410.

253. Britten RA, Duncan VD, Fesshaye A, Rudobeck E, Nelson GA, Vlkolinsky R. Altered Cognitive Flexibility and Synaptic Plasticity in the Rat Prefrontal Cortex after Exposure to Low ( $\leq 15$ cGy) Doses of (28)Si Radiation. Radiation research. 2020;193(3):223-35 . 


\title{
Appendix \\ Appendix A: Abstracts from first authored original research manuscripts
}

\author{
RNA-sequencing and behavioral testing reveals inherited physical inactivity co- \\ selects for anxiogenic behavior without altering depressive-like behavior in Wistar \\ rats
}

Taylor J Kelty, Jacob D Brown, Nathan R Kerr, Michael D Roberts, Tom E Childs, Omar H Cabrera, Francesca M Manzella, Dennis K Miller, George T Taylor, Frank W Booth

Neuroscience Letters, May 2021; Volume 753

Physical inactivity is positively associated with anxiety and depression. Considering physical inactivity, anxiety, and depression each have a genetic basis for inheritance, our lab used artificial selectively bred low-voluntary running (LVR) and wild type (WT) female Wistar rats to test if physical inactivity genes selected over multiple generations would lead to an anxiety or depressive-like phenotype. We performed next generation RNA sequencing and immunoblotting on the dentate gyrus to reveal key biological functions from heritable physical inactivity. LVR rats did not display depressive-like behavior. However, LVR rats did display anxiogenic behavior with gene networks associated with reduced neuronal development, proliferation, and function compared to WT counterparts. Additionally, immunoblotting revealed LVR deficits in neuronal development and function. To our knowledge, this is the first study to show that by selectively breeding for physical inactivity genes, anxiety-like genes were co-selected. The study also reveals molecular insights to the genetic influences that physical inactivity has on anxiety-like behavior. 


\section{Appendix B: Abstracts from co-authored original research manuscripts}

\section{Maternal Western diet age-specifically alters female offspring voluntary physical activity and dopamine- and leptin-related gene expression}

Gregory N Ruegsegger, Kolter B Grigsby, Taylor J Kelty, Terese M Zidon, Thomas E Childs, Victoria J Vieira-Potter, David L Klinkebiel, Michael Matheny, Phillip J Scarpace, Frank W Booth

The FASEB Journal, Aug 2017; Volume 31: 5371-5383

Prenatal overnutrition affects development into adulthood and influences risk of obesity. We assessed the transgenerational effect of maternal Western diet (WD) consumption on offspring physical activity. Voluntary wheel running was increased in juvenile (4-7 wk ofage), but decreased in adult (16-19 wk of age), $F_{1}$ female WD offspring. In contrast, no wheel-running differences in $F_{1}$ male offspring were observed. Increased wheel running in juvenile female WD offspring was associated with up-regulated dopamine receptor (DRD)-1 and -2 in the nucleus accumbens (NAc) and with down-regulated Leprin the ventral tegmental area (VTA). Conversely, decreased wheel running by adult female WD offspring was associated with down-regulated DRD1 in the NAc and with up-regulated Lepr in the VTA. Body fat, leptin, and insulin were increased in male, but not in female, $F_{1}$ WD offspring. Recombinant virus (rAAV) leptin antagonism in the VTA decreased wheel running in standard diet but not in WD $F_{1}$ female offspring. 
Analysis of $F_{2}$ offspring found no differences in wheel running or adiposity in male or female offspring, suggesting that changes in the $F_{1}$ generation were related to in utero somatic reprogramming. Our findings indicate prenatal WD exposure leads to age-specific changes in voluntary physical activity in female offspring that are differentially influenced by VTA leptin antagonism.-Ruegsegger, G. N., Grigsby, K. B., Kelty, T. J., Zidon, T. M., Childs, T. E., Vieira-Potter, V. J., Klinkebiel, D. L., Matheny, M., Scarpace, P. J., Booth, F. W. Maternal Western diet age-specifically alters female offspring voluntary physical activity and dopamine- and leptin-related gene expression. 


\section{Medial habenula maturational deficits associate with low motivation for voluntary physical activity}

Kolter B Grigsby, Taylor J Kelty, Frank W Booth

Brain Research, Nov 2018; Volume 1698: 187-194

The habenula is a small, diencephalic structure comprised of distinct subnuclei which receives inputs from the limbic forebrain and sends projections to various regions in the midbrain, making this region well positioned to influence reward and motivation. Genetic ablation of the dorsal medial habenula is known to decrease voluntary wheel-running in mice. However, the extent to which the medial habenula $(\mathrm{MHb})$ mediates wheel-running motivation in the context of high or low motivation for voluntary physical activity remains to be determined. In so, we utilized 5-week-old female rats selectively bred to voluntarily run high (HVR) or low (LVR) distances in order to determine if inherent differences in medial habenula maturation accompany inherent differences in wheel-running motivation. We report a significantly higher expression of genes associated with $\mathrm{MHb}$ development (Brn3a, Nurr1, Tac1, and Kcnip) in HVR versus LVR rats. Furthermore, there was a positive correlation between Brn3a and Nurr1 expression and run distance in HVR, but

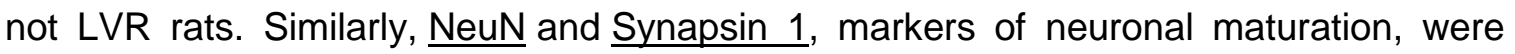
higher in HVR compared to LVR rats. Lastly, dendritic density was determined to be higher in the MHb of HVR versus LVR rats, while LVR rats showed a higher percentage of thin spines, suggesting a higher prevalence of immature dendrites in LVR rats. Taken together, the above findings highlight the involvement of $\mathrm{MHb}$ in driving the motivation to be physically active. Given pandemic levels of global physical inactivity, the role of the $\mathrm{MHb}$ offers a novel potential to improve our global health. 


\section{Ketogenic diet in combination with voluntary exercise impacts markers of hepatic metabolism and oxidative stress in male and female Wistar rats}

Mary P Moore, Rory P Cunningham, Taylor J Kelty, Luigi R Boccardi, Nhu Y Nguyen, Frank W Booth, R Scott Rector

Applied Physiology, Nutrition, and Metabolism, Jan 2020; Volume 45: 1-10

Ketogenic diets (KDs) are shown to benefit hepatic metabolism; however, their effect on the liver when combined with exercise is unknown. We investigated the effects of a KD versus a "western" diet (WD) on markers of hepatic lipid metabolism and oxidative stress in exercising rats. Male and female Wistar rats with access to voluntary running wheels were randomized to three groups ( $n=8-14$ per group): standard chow (SC; $17 \%$ fat), WD (42\% fat), or KD (90.5\% fat) for seven weeks. Body fat percentage (BF\%) was increased in $\mathrm{WD}$ and $\mathrm{KD}$ versus $\mathrm{SC}$, although KD females displayed lower BF\% versus WD ( $p \leq$ 0.05). Liver triglycerides were higher in $K D$ and $W D$ versus $S C$ but were attenuated in $K D$ females versus WD $(p \leq 0.05)$. KD suppressed hepatic markers of de novo lipogenesis (fatty acid synthase, acetyl coenzyme A carboxylase) and increased markers of mitochondrial biogenesis/content (peroxisome proliferator activated receptor-1a, mitochondrial transcription factor A (TFAM), and citrate synthase activity). KD also increased hepatic glutathione peroxidase one and lowered oxidized glutathione. Female rats exhibited elevated hepatic markers of mitochondrial biogenesis (TFAM), mitophagy (light chain three II/I ratio, autophagy-related protein 12:5), and cellular energy homeostasis (phosphorylated 5'AMP-activated protein kinase/5'AMP-activated protein kinase) versus males. These data highlight that $K D$ and exercise beneficially affects hepatic metabolism and oxidative stress and merits further investigation. 


\section{Appendix C: Presented abstracts outside of dissertation topics}

\section{Western Diet (Wd) and Ketogenic Diet (Kd) Implications on Voluntary Wheel Running}

Taylor J. Kelty , Kolter B. Grigsby, Gregory N. Ruegsegger, Frank W. Booth

Physical inactivity is closely associated with a multitude of chronic diseases, which can lead to a poorer quality of life and premature death. The mesolimbic dopamine system, specifically the nucleus accumbens (NAc), has been shown to modulate motivation and reward pathways that drive physical activity. Opioids help modulate this system by regulating dopamine release. High fat diets (HFD) are known to alter pathways in the brain that drive physical inactivity. However, underlying mechanisms still need more exploration. Objective: The purpose of my study was to exam HFD's effect on physical activity. Methods: In this study Wister rats at seven weeks of age 1) were placed into running wheels and 2) fed a ketogenic diet (KD), western diet (WD), or standard chow diet (SD) chronically for seven weeks. Previous studies from the literature shows rats on a HFD tend to overeat leading to weight gain that may lead to decreased voluntary running. In order to measure physical activity levels independently of caloric intake, rats on the KD and WD were only allowed access to same amount of food calories consumed by the SD group. Results: Despite giving equal caloric amounts, male rats on the KD had attenuated weight gain $(p \leq 0.001)$ compared to rats fed SD after the first week on the diet. Both WD and KD animals had a larger percentage body fat on either HFD compared to SD ( $p \leq$ 0.01). Male rats fed either a KD or WD had overall increases in voluntary wheel running $(p \leq 0.05)$ compared to the SD group. Upon molecular examination of the NAc the KD 
group had higher expression of opioid receptor kappa one (oprk1) $(p \leq 0.05)$. These data suggest that the observed increase in running in the KD group could be partially due to alterations in the opioid system. Conclusion: HFD's alter voluntary running in which male Wistar rats showed an increase in voluntary wheel running which could be partially due to alterations by the opioid system. Significance: This study provides specific novel insights on how the KD and WD might change physical activity, which will help combat physical inactivity to increase quality and quantity of lifespan. 


\section{Ladder climbing ameliorates LPS-induced cognitive impairment in resistance- exercise trained (RT) rats}

Taylor J. Kelty, Xuansong Mao, Kolter B. Grigsby, Tom Childs, Paige N. Michener, Rachel A. Richardson, Todd R. Schachtman, and Frank W. Booth

Introduction: Alzheimer's disease is the most prevalent form of dementia affecting nearly six million Americans. Since no cure for Alzheimer's disease exists, it is imperative to develop therapies that can prevent or delay its progression. Resistance training (RT) can ameliorate mild cognitive impairment (MCl), the forerunner of Alzheimer's disease. RT activates IGF-1 signaling that is associated with neuronal proliferation, survival, and plasticity. Purpose: Therefore, the purpose of this study is to fill a knowledge gap that connects RT and IGF-1 signaling in a model of MCl. Methodology: To induce MCl, 4.5month-old male Wistar rats were underwent intracerebroventricular (ICV) lipopolysaccharide (LPS) injections, bacteria that causes chronic neuroinflammation. Following induction of $\mathrm{MCl}$, rats underwent 6-weeks of progressive RT by climbing a ladder with weights attached to their tails. During the last week of training, rats went through behavioral testing to measure cognition. Rat were sacrificed and their dentate gyrus was removed to quantify changes in markers of neuroplasticity and IGF-1 signaling. Results: RT rats showed amelioration of cognition, increased IGF-1 receptor transcript, increased downstream IGF-1 signaling, and increased neuroplasticity marker protein synapsin one compared to $\mathrm{MCl}$-induced counterparts. Conclusion: Overall, these data suggest that $\mathrm{RT}$ is able to ameliorate $\mathrm{MCl}$ that is associated with increased IGF-1 signaling. 


\section{Transcriptomic examination of inherited genes of physical inactivity on anxiety-like behavior in Wistar rats}

Taylor J. Kelty, Jacob D. Brown, Michael D. Roberts, Tom E. Childs, Omar H. Cabrera, Francesca M. Manzella, Dennis K. Miller, George T. Taylor, and Frank W. Booth

Introduction: Despite studies showing physical activity to be anxiolytic, physical activity levels have steadily declined in high-income countries, suggesting an increased vulnerability to anxiety. Genetic factors influence both anxiety disorders and physical activity. Methods: Combining the genetic influences of physical inactivity with the increased prevalence of anxiety, our lab used selectively bred low voluntary wheel running (LVR) or wild type (WT) Wistar rats to test if physical inactivity genes selected over multiple generations would lead to an anxiogenic phenotype. Rats were also given access to running wheels to discover any molecular changes associated with voluntary wheel running that could be anxiolytic in this phenotype. In addition, we employed a chronic mild stress (CMS) model to determine if LVR rats were more susceptible to the development of anxiogenic behavior. With the use of next generation sequencing, we performed RNA sequencing on the dentate gyrus of these rats to reveal key pathways involved in anxiety. Results: LVR rats had increased anxiogenic behavior compared to WT rats. While voluntary wheel running proved to be anxiolytic after exposure to CMS. Dentate gyrus bioinformatics revealed that LVR rats have gene networks associated with reduced neuronal proliferation and function. Conclusion: To our knowledge, this is the first study to show that by selectively breeding for physical inactivity genes, anxiety genes were coselected with genes associated with reduced neuronal proliferation and function in the dentate gyrus. 


\section{VITA}

Taylor Jacob Kelty was born in September of 1993 in the small town of Quincy, Illinois to parents, Thomas and Deanna Kelty. Taylor graduated from Quincy University with his Bachelors of Science (B.S.) in biology and chemistry in May of 2016. In the summer of 2016, he went on to graduate school at University of Missouri in the Department of Biomedical Sciences where he trained under his mentor, Dr. Frank Booth, studying neuromolecular adaptions from exercise. After five years, Taylor defended his dissertation thesis and accepted a postdoctoral fellowship position under Dr. Scott Rector at University of Missouri School of Medicine in the Department of Nutrition and Exercise Physiology, where he will continue his training by studying the integrative physiological adaptions from exercise. 